\title{
Decoupling of supersymmetric particles
}

\author{
A. Dobado ${ }^{1, a}$, M.J. Herrero ${ }^{2, b}$, S. Peñaranda ${ }^{2, c}$ \\ 1 Departamento de Física Teórica, Universidad Complutense de Madrid, E-28040 Madrid, Spain \\ 2 Departamento de Física Teórica, Universidad Autónoma de Madrid, Cantoblanco, E-28049 Madrid, Spain
}

Received: 27 March 1998 / Published online: 5 October 1998

\begin{abstract}
The possibility of a heavy supersymmetric spectrum at the Minimal Supersymmetric Standard Model is considered and the decoupling from the low energy electroweak scale is analyzed in detail. The formal proof of decoupling of supersymmetric particles from low energy physics is stated in terms of the effective action for the particles of the Standard Model that results by integrating out all the sparticles in the limit where their masses are larger than the electroweak scale. The computation of the effective action for the standard electroweak gauge bosons $W^{ \pm}, Z$ and $\gamma$ is performed by integrating out all the squarks, sleptons, charginos and neutralinos to one-loop. The Higgs sector is not considered in this paper. The large sparticle masses limit is also analyzed in detail. Explicit analytical formulae for the two-point functions of the electroweak gauge bosons to be valid in that limit are presented. Finally, the decoupling of sparticles in the $S, T$ and $U$ parameters is studied analitically. A discussion on how the decoupling takes place in terms of both the physical sparticle masses and the non-physical mass parameters as the $\mu$-parameter and the soft-breaking parameters is included.
\end{abstract}

\section{Introduction}

The Standard Model (SM) of particle physics provides a highly successful description of all particle physics phenomena occurring at present day accelerators. No experimental deviations from the SM have yet been found, even at LEP where the measurement of precision observables provides a very sensitive test to possible deviations through radiative corrections. Therefore, it is clear that the SM is an extremely accurate effective low energy theory at energy scales up to, at least, $100 \mathrm{GeV}$. In spite of this success there is, however, the strong belief among particle physicists that the SM cannot be the ultimate theory but it must be the low energy effective theory of some other more fundamental one which will probably incorporate gravitational interactions. Among the various extensions of the SM, the Supersymmetric (SUSY) theories are the most claimed ones $[1,2]$. The motivations have been principally theoretical. The idea of supersymmetry relating fermions and bosons is certainly beautiful itself, but one of the most used arguments in favour of supersymmetry is that it guarantees the absence of quadratic divergences in scalar self-energies which, in turn, keeps the wanted stability of widely separated electroweak and grand unified scales. In practice, the supersymmetric theories are mostly built within same grand unification scenario and have as a common prediction the existence of, at least, one light elementary scalar Higgs boson with a mass

\footnotetext{
a e-mail: dobado@eucmax.sim.ucm.es

b e-mail: herrero@delta.ft.uam.es

c e-mail: siannah@delta.ft.uam.es
}

close to the electroweak scale. At present, both aspects the idea of grand unification and the existence of a light Higgs boson are still waiting for experimental confirmation.

However, the greatest dilemma for supersymmetry is the unknown mechanism for supersymmetry breaking. Since no supersymmetric partners of the known particles have yet been observed, one must not only explain why supersymmetry is broken, but also why it is broken in such a way that all the supersymmetric partners are heavier than the known particles. The present experimental limits on the sparticle masses [3] indicate that supersymmetry must be indeed strongly broken. In practice, when building a supersymmetric theory for low energy physics one typically avoids the issue of the exact mechanism of supersymmetry breaking and parametrizes this phenomenon by introducing a set of supersymmetry breaking parameters which characterize the scale of the sparticle masses. These explicit SUSY breaking terms are required, in addition, to be of soft type, meaning that no new quadratic divergences are generated in the broken SUSY theory [4]. In other to keep the wanted shielding of the electroweak scale against potentially large corrections from the grand unified scale, at least at a phenomenologically acceptable level, these soft SUSY breaking mass parameters shouldn't be larger than say $O(1 T e V)$. This condition translates into a restriction on the largest allowed mass splitting between the known particles and their superpartners of this same order of magnitude and one concludes that the supersymmetric particles cannot be heavier than say $O(1 T e V)$. The simplest low energy supersymmetric model that includes these soft SUSY breaking terms is the Minimal Supersym- 
metric Standard Model (MSSM) [5], which is the one we have chosen to work with in this paper.

One interesting aspect that arises in these softly broken SUSY theories, and in particular in the MSSM, is the question of decoupling of heavy sparticles from the low energy SM and how does it really occurs if it occurs at all. This is the main subject of the present paper. It is a common belief that when the spectrum of supersymmetric particles is considered much heavier than the low energy electroweak scale they decouple from the low energy physics, even at the quantum level, and the resulting low energy effective theory is the SM itself. It is true that there are indications that this will happen, but a rigorous proof of decoupling is still lacking. On one hand there are numerical studies of observables that measure electroweak radiative corrections, like $\Delta r$ and $\Delta \rho[6]$, or the $S, T$ and $U$ parameters [7] as well as in the $Z$ boson, top quark and Higgs decays [8], which indicate that the one loop corrections from supersymmetric particles decrease up to negligible values in the limit of very heavy sparticle masses. On the other hand there are some analytical studies, in certain asymptotic limits, of these and related observables [6]-[10], as well as some computations of the effective potential for the scalar sector [12], where one of the mass parameters like the so-called $\mu$-parameter or the soft-SUSY breaking parameters are pushed to infinity. They also indicate some kind of decoupling but they are not completely general since they mostly assume, for simplicity, the restrictive hypothesis of universality of the soft SUSY breaking parameters. Besides, one must be aware that not all these asymptotic limits in terms of the mass scale parameters of the MSSM lead to a complete heavy SUSY spectra. In order to study the true decoupling, one must be careful in taking the proper asymptotic limits such that all the sparticles, and not just some of them, are got heavier than their superpartners. In other words, the choices of SUSY parameters that lead to some heavy and some light SUSY particles are not the proper ones to study the decoupling hypothesis, since the presence of new light spectra at low energies is by itself contradictory with the idea of decoupling. Therefore to explore the decoupling phenomenon it is more convenient to study the asymptotic behaviour of the theory in the large sparticle physical masses limit themselves rather than in the large MSSM mass parameters limit. In this spirit there are some works which deal with the Higgs sector of the MSSM and study the consequences of taking one of the Higgs particles infinitely heavy. In particular, when the mass of the pseudoscalar boson $A^{o}$ is pushed to infinity it is proved in [11] that, up to one loop level, all the effects of the heavy Higgs particles decouple from the electroweak precision observables. More specifically, in this limit four of the Higgs particles, $H^{ \pm}, H^{o}$ and $A^{o}$ become infinitely heavy and the fifth one, $h^{o}$, remains close to the electroweak scale. The unique trace of the SUSY Higgs sector that is left to low energies is the unavoidable presence of the light Higgs particle $h^{o}$ itself which turns out to be, in the above limit, indistinguishable from the Higgs particle of the SM. In this sense, it is concluded in [11] that there is indeed decoupling in the Higgs sector of the MSSM. Notice, however, that this behaviour is generic of the MSSM, but in more extended Higgs sectors and in non-supersymmetric models with two Higgs doublets it is known that the decoupling of the heavy Higgs particles may not occur [13].

A formal proof of decoupling must be driven, however, along the lines stated in the famous Decoupling Theorem of Appelquist and Carazzone [14]. The theorem states that under certain conditions in a given Quantum Field Theory with light and heavy particles, if the heavy particles are integrated out to all orders in perturbation theory, the remaining effective action to be valid at energies much lower that the heavy particle masses does not show any trace of these heavy particles. In that case they are said to decouple from the low energy theory. More specifically, all the quantum effects of the heavy particles that are left in the effective action can be either absorbed into a redefinition of the parameters of the original theory or wave function renormalization referring to the light fields, or they are suppressed by inverse powers of the heavy masses and, therefore, vanish in the infinite mass limit. Among the conditions on the starting field theory to be sure that the theorem holds are the renormalizability and the absence of spontaneous symmetry breaking and chiral fermions. Examples where the Decoupling Theorem does not hold are well known. Particularly interesting are the cases of the Higgs particle and the top quark in the SM which are known not to decouple from low energy physics [16]. For instance, by integrating out the top quark at one loop one finds new contributions in the effective action which do not vanish in the infinite top mass limit [17]. The same occurs when integrating out the Higgs particle at one-loop. [18] This can also be seen in the observable $\Delta \rho$ where the dominant contribution from the top quark loops in the large top mass limit goes quadratically with the top mass and the dominant contribution from the Higgs particle loops in the large Higgs mass limit goes logarithmically with the Higgs mass. In these examples one understands the departure from the Decoupling Theorem because the SM is a gauge theory with both spontaneous symmetry breaking and chiral fermions [19].

The question whether the Decoupling Theorem applies or not in the case of heavy sparticles in the MSSM is not obvious at all, in our opinion. The MSSM is a model which incorporates the SM particle content, the SM gauge interactions and it is built such that the wanted spontaneous breaking pattern, $S U(2)_{\mathrm{L}} \times U(1)_{\mathrm{Y}} \rightarrow U(1)_{\mathrm{em}}$, takes place as in the Electroweak Theory. Even more, the softSUSY breaking terms are required not just to generate the needed SUSY particle masses but in addition to allow for this spontaneous electroweak symmetry breaking [20]. In few words, the MSSM is a gauge theory, as the SM, with spontaneous symmetry breaking and chiral fermions and therefore, the direct application of the Decoupling Theorem should, in the principle, be questioned.

In our opinion, a formal proof of decoupling must involve the explicit computation of the effective action by integrating out one by one all the sparticles in the MSSM to all orders in perturbation theory, and by considering the 
heavy sparticle masses limit. The proof will be conclusive if the remaining effective action, to be valid at energies much lower than the SUSY particle masses, turns out to be that of the SM with all the SUSY effects being absorbed into a redefinition of the SM parameters or else they are suppressed by inverse powers of the SUSY particle masses and vanish in the infinite masses limit.

In the present paper we give a first step in this direction and compute the two-point functions part of the effective action which results by integrating out all the SUSY particles of the MSSM, except the Higgs sector, at the one loop level. The integration of the Higgs sector presents interesting features by itself and we have preferred to consider it separately in a forthcoming work [21]. Of course, the complete proof of decoupling must involve the computation of all the n-point Green functions. The part of the effective action we have chosen to start with is the one for the electroweak gauge bosons, $W^{ \pm}, \mathrm{Z}$ and $\gamma$ and, in particular, we have derived here the two point functions. The three and four point functions with external $W^{ \pm}, \mathrm{Z}$ and $\gamma$ will be analized in a different work [22]. From the two-point functions we will derive, in addition, the contributions from the SUSY particles to the $S, T$ and $U$ parameters in the large SUSY masses limit. From these analytical expressions we will be able to conclude how the decoupling really occurs in these parameters. In order to keep our computation of the heavy SUSY particle quantum effects in a general form we have chosen to work with the masses themselves. They are the proper parameters of the large mass expansions instead of another more model dependent choices as the $\mu$-parameter or the softSUSY-breaking parameters. We have considered the physically plausible situation where all the sparticle masses are large as compared to the electroweak scale but they are allowed, in principle, to be different from each other. We will explore the interesting question of to what extent the usual hypothesis of SUSY masses being generated by softSUSY- breaking terms and the universality of the mass parameters do or do not play a relevant role in getting decoupling. In fact, we will show in this paper, that the basic requirement of $S U(2)_{\mathrm{L}} \times U(1)_{\mathrm{Y}}$ gauge invariance on the SUSY breaking terms is sufficient to obtain decoupling in the MSSM.

Finally, we have dedicated special attention and have been very careful in evaluating analytically the large SUSY masses limit of the Green functions. For this purpose, we have applied the so-called m-Theorem [23] which provides a rigorous technique to compute Feynman integrals with both large and small masses in the asymptotic limit $\tilde{m}_{i} \rightarrow \infty$ for the large masses.

The paper is organized as follows: The second section contains a short review of the MSSM and the supersymmetric spectra and fixes the notation. The large sparticle masses limit is described in section three. A discussion on how to get large mass values for all the squarks, sleptons, neutralinos and charginos in the MSSM is also included. The fourth section is devoted to the computation of the effective action for the electroweak gauge bosons $W^{ \pm}, \mathrm{Z}$ and $\gamma$ in the MSSM that results by integrating out, in the path integral, squarks, sleptons, charginos and neutralinos to one-loop. The exact results to one-loop for the two-point functions of the electroweak gauge bosons are presented in the fifth section. A discussion on the application of the m-Theorem and the analytical results for the self-energies of the electroweak gauge bosons in the large SUSY masses limit are also included and commented in this section. The decoupling of heavy sparticles in the $S, T$ and $U$ parameters is analyzed in section six. Explicit formulae for these parameters in the large SUSY masses limit as well as a discussion on these results are also presented in this section. The conclusions are summarized in section seven. Appendix A contains the exact results to one-loop of the self-energies, $\Sigma^{X Y}$, and the part of the two-point functions that is proportional to $k_{\mu} k_{\nu}, R^{X Y}$. The evaluation of the relevant Feynman integrals in the large masses limit and the application of the m-Theorem are performed in Appendix B. The asymptotic results in the above limit for the transverse and longitudinal parts of the two-point functions, $\Sigma_{T}^{X Y}$ and $\Sigma_{L}^{X Y}$, are collected in Appendix C.

\section{Some remarks on the MSSM}

The Minimal Supersymmetric extension of the Standard Model consists of taking the Standard Model of electroweak and strong interactions as it is known today, including the as yet undiscovered Higgs boson particle, and adding the corresponding supersymmetric partners $[2,5]$.

The supersymmetric partners of the quarks and leptons are the spin-zero squarks and sleptons. For a given fermion $f$, there are two supersymmetric partners $\tilde{f}_{L}$ and $\tilde{f}_{R}$ which are the scalar partners of the corresponding left and right-handed fermions. Correspondingly, in the case of sneutrinos there is no $\tilde{\nu}_{R}$. For simplicity we shall ignore intergenerational mixing.

The supersymmetric partners of the gauge bosons are the spin-one-half fermions called gauginos. The partners of the gluon $g_{\mu}$, and the four weak bosons $W_{\mu}^{a}(\mathrm{a}=1,2,3)$, $B_{\mu}$ are, correspondingly, the gluino $\tilde{g}$, the winos $\tilde{W}^{a}$ and the bino $\tilde{B}$.

In addition, the MSSM must possess two complex scalar Higgs doublets $H_{1}, H_{2}$ in order to give masses to down and up type fermions in a manner consistent with supersymmetry. The corresponding fermionic superpartners are the Higgsinos $\tilde{H}_{1}, \tilde{H}_{2}$ which are also needed in pairs, in order to avoid gauge anomalies. Supersymmetry, on the other hand, imposes strong constraints on the form of the Higgs potential. In particular, the quartic self couplings of the Higgs fields are fixed in terms of the $S U(2) \times U(1)$ gauge couplings and, therefore, the Higgs sector of the MSSM is always weakly interacting.

The MSSM Lagrangian consists of two parts, a supersymmetry-conserving Lagrangian and a supersymmetrybreaking Lagrangian:

$$
\mathcal{L}_{\mathrm{MSSM}}=\mathcal{L}_{\mathrm{SUSY}}+\mathcal{L}_{\mathrm{SUSY}}^{\mathrm{break}}
$$


Usually $\mathcal{L}_{\mathrm{MSSM}}$ is required to contain no interaction terms of higher dimensions than 4 so that the theory is renormalizable.

$\mathcal{L}_{\text {SUSY }}$ contains the SM Lagrangian for the gauge bosons and fermions and the corresponding supersymmetrized Lagrangian for the gauginos and sfermions. It also includes all possible renormalizable interactions among sparticles themselves and among particles and sparticles which are compatible with $S U(3)_{\mathrm{c}} \times S U(2)_{\mathrm{L}} \times U(1)_{\mathrm{Y}}$ gauge invariance and with supersymmetry. In addition, there are the Lagrangian of the Higgs sector and the corresponding supersymmetrized Lagrangian for the Higgsino sector. This Higgs/Higgsino part includes the self-interaction terms and also the interactions with the other sectors. In particular it contains the Yukawa interactions with the standard fermions and the supersymmetric Higgs potential:

$$
\begin{aligned}
V_{\mathrm{Higgs}}^{\mathrm{SUSY}}= & |\mu|^{2}\left(\left|H_{1}\right|^{2}+\left|H_{2}\right|^{2}\right) \\
& +\frac{1}{8}\left(g^{2}+g^{\prime 2}\right)\left(\left|H_{1}\right|^{2}-\left|H_{2}\right|^{2}\right)^{2}+\frac{1}{2} g^{2}\left|H_{1}^{*} H_{2}\right|^{2}
\end{aligned}
$$

The supersymmetry-conserving Lagrangian, $\mathcal{L}_{\mathrm{SUSY}}$, is completely general and model-independent since it is based just in $S U(3)_{\mathrm{c}} \times S U(2)_{\mathrm{L}} \times U(1)_{\mathrm{Y}}$ gauge symmetry, renormalizability and supersymmetry. The parameters of $\mathcal{L}_{\text {SUSY }}$ can be summarized as follows:

- the gauge couplings $g_{s}, g$ and $g^{\prime}$ corresponding to the SM gauge group $S U(3)_{\mathrm{c}} \times S U(2)_{\mathrm{L}} \times U(1)_{\mathrm{Y}}$ respectively, - the Higgs Yukawa couplings $\lambda_{e}, \lambda_{u}$ and $\lambda_{d}$ (which are $3 \times 3$ matrices in flavour space), and

- the supersymmetry-conserving mass parameter $\mu$.

Concerning the SUSY-breaking part, $\mathcal{L}_{\mathrm{SUSY}}^{\text {break }}$, it is not of a general form but its detailed form and the involved parameters depend on the particular mechanism that is chosen in order to produce the SUSY breaking. However, we are forced to introduce some kind of explicit SUSY breaking at low energies since no supersymmetric partners of the known particles have been observed.

In order to study the decoupling of SUSY particles we would like to consider this term in the most model independent way as possible. For this purpose, the minimal requirements that $\mathcal{L}_{\mathrm{SUSY}}^{\text {break }}$ must fulfil are: 1) $S U(3)_{\mathrm{c}} \times$ $S U(2)_{\mathrm{L}} \times U(1)_{\mathrm{Y}}$ gauge invariance, and 2$)$ it must transmit masses to all the supersymmetric particles in a way that these result being considerably heavier than their standard partners. This last point is needed since we wish to study the consequences at low energies of having a gap between the SUSY spectra and the SM spectra.

Here we shall adopt the low-energy point of view in which one skirts the issue of the exact mechanism of SUSY breaking, and parametrizes one's ignorance by introducing general mass-SUSY-breaking parameters that give the scale of the masses of the SUSY particles without inquiring into its origins. Usually, these explicit SUSY breaking terms are required, in addition to be of soft type, meaning that no new quadratic divergences are generated by the explicitly broken SUSY theory. These soft breaking terms were classified by Girardello and Grisaru [4] and are of four types: gaugino Majorana mass terms, scalar mass terms, scalar-scalar-scalar trilinear interaction terms, and scalar-scalar bilinear interaction terms.

In the case of the MSSM the set of all possible soft terms that respect $S U(3)_{\mathrm{c}} \times S U(2)_{L} \times U(1)_{Y}$ gauge invariance are listed below:

$$
\begin{aligned}
V_{\text {soft }}= & m_{1}^{2}\left|H_{1}\right|^{2}+m_{2}^{2}\left|H_{2}\right|^{2}-m_{12}^{2}\left(\epsilon_{i j} H_{1}^{i} H_{2}^{j}+\text { h.c. }\right) \\
& +M_{\tilde{Q}}^{2}\left[\tilde{t}_{L}^{*} \tilde{t}_{L}+\tilde{b}_{L}^{*} \tilde{b}_{L}\right]+M_{\tilde{U}}^{2} \tilde{t}_{R}^{*} \tilde{t}_{R}+M_{\tilde{D}}^{2} \tilde{b}_{R}^{*} \tilde{b}_{R} \\
& +M_{\tilde{L}}^{2}\left[\tilde{\nu}_{L}^{*} \tilde{\nu}_{L}+\tilde{\tau}_{L}^{*} \tilde{\tau}_{L}\right]+M_{\tilde{E}}^{2} \tilde{\tau}_{R}^{*} \tilde{\tau}_{R} \\
& +\frac{g}{\sqrt{2} m_{W}} \epsilon_{i j}\left[\frac{m_{\tau} A_{\tau}}{\cos \beta} H_{1}^{i} \tilde{l}_{L}^{j} \tilde{\tau}_{R}^{*}\right. \\
& \left.+\frac{m_{b} A_{b}}{\cos \beta} H_{1}^{i} \tilde{q}_{L}^{j} \tilde{b}_{R}^{*}-\frac{m_{t} A_{t}}{\sin \beta} H_{2}^{i} \tilde{q}_{L}^{j} \tilde{t}_{R}^{*}\right] \\
& +\frac{1}{2}\left[M_{3} \overline{\tilde{g}} \tilde{g}+M_{2} \overline{\tilde{W}}^{a} \tilde{W}^{a}+M_{1} \overline{\tilde{B}} \tilde{B}\right]
\end{aligned}
$$

where,

$$
\tilde{l}_{L} \equiv\left(\begin{array}{c}
\tilde{\nu}_{L} \\
\tilde{\tau}_{L}
\end{array}\right), \tilde{q}_{L} \equiv\left(\begin{array}{c}
\tilde{t}_{L} \\
\tilde{b}_{L}
\end{array}\right),
$$

$\epsilon_{i j}(i, j=1,2)$ is the antisymmetric tensor to combine two $S U(2)_{L}$ doublets $\left(\epsilon_{12}=1\right)$ and the third generation notation is used. The generation labels that are omitted for brevity in this formula must be assumed.

The parameters of $V_{\text {soft }}$ can be summarized as follows:

- three scalar Higgs mass parameters $m_{1}^{2}, m_{2}^{2}$, and $m_{12}^{2}$, with $m_{12}^{2} \equiv B \mu$ where $\mu$ is the mass parameter introduced in (2) and $B$ is a soft breaking parameter. These can be re-expressed in terms of the two Higgs vacuum expectation values, $v_{1}$ and $v_{2}$, and one physical Higgs mass. Here, $v_{1}\left(v_{2}\right)$ is the vacuum expectation value of the Higgs field which couples exclusively to downtype (up-type) quarks and leptons. Since $v_{1}^{2}+v_{2}^{2}=$ $(246 \mathrm{GeV})^{2}$ is fixed by the $W$ mass, we are left with just two independent parameters in the Higgs sector. These are usually chosen to be $\tan \beta=v_{2} / v_{1}$ and the mass of the pseudoscalar $m_{A^{0}}^{2}=m_{12}^{2}(\tan \beta+\cot \beta)$,

- scalar masses for the squarks and sleptons of each generation: $M_{\tilde{Q}}, M_{\tilde{U}}, M_{\tilde{D}}, M_{\tilde{L}}$ and $M_{\tilde{E}}$,

- the trilinear soft breaking parameters for sleptons and squarks of each generation $A_{r}, A_{t}$ and $A_{b}$, and finally,

- gaugino Majorana masses $M_{3}, M_{2}$ and $M_{1}$ associated with the $S U(3)_{\mathrm{c}}, S U(2)_{\mathrm{L}}$ and $U(1)_{\mathrm{Y}}$ subgroups of the $\mathrm{SM}$ respectively.

At this point we would like to make a short comment that we think is relevant for the discussion about decoupling which will be presented in this paper. Notice that the mass terms for gauginos and scalar particles above are the unique possible explicit mass terms that are compatible with $S U(3)_{\mathrm{c}} \times S U(2)_{\mathrm{L}} \times U(1)_{\mathrm{Y}}$ gauge invariance. For instance, we have chosen not to put different mass terms for the two members of a scalar $S U(2)_{\mathrm{L}}$ doublet, and we have not included Dirac fermion mass terms. Any of these two types of terms would have broken the weak isospin symmetry and consequently the $S U(2)_{L}$ gauge symmetry 
of the Lagrangian. Thus, the above mass terms are completely general and could have been introduced without mentioning the soft breaking requirement, but just the $S U(3)_{\mathrm{c}} \times S U(2)_{\mathrm{L}} \times U(1)_{\mathrm{Y}}$ gauge invariance. In the following we will refer to these general terms simply as SUSY breaking mass terms. On the other hand, it is known that the SUSY breaking part of the Lagrangian is needed in order to incorporate the wanted spontaneous breaking of $S U(2)_{\mathrm{L}} \times U(1)_{\mathrm{Y}}$ into $U(1)_{\mathrm{em}}$. It is not possible to break spontaneously the $S U(2)_{\mathrm{L}} \times U(1)_{\mathrm{Y}}$ symmetry if the theory has an exact supersymmetry. The reason is that the supersymmetric Higgs potential in (2) is definite positive and its minimum is at the symmetric configuration $H_{1}=H_{2}=0$. Once the SUSY-breaking Higgs mass parameters, $m_{1}^{2}, m_{2}^{2}$ and $m_{12}^{2}$, are included in the Higgs potential it reads:

$$
\begin{aligned}
V_{\text {Higgs }}= & m_{1 H}^{2}\left|H_{1}\right|^{2}+m_{2 H}^{2}\left|H_{2}\right|^{2}-m_{12}^{2}\left(\epsilon_{i j} H_{1}^{i} H_{2}^{j}+\text { h.c. }\right) \\
& +\frac{1}{8}\left(g^{2}+g^{\prime 2}\right)\left(\left|H_{1}\right|^{2}-\left|H_{2}\right|^{2}\right)^{2} \\
& +\frac{1}{2} g^{2}\left|H_{1}^{*} H_{2}\right|^{2}
\end{aligned}
$$

where $m_{i H}^{2} \equiv|\mu|^{2}+m_{i}^{2}(\mathrm{i}=1,2)$, and the real parameters $m_{i}^{2}$ can be either positive or negative, therefore allowing $V_{\text {Higgs }}$ to develop non-trivial vacua. The parameter region in which $S U(2)_{\mathrm{L}} \times U(1)_{\mathrm{Y}}$ breaks down to $U(1)_{\mathrm{em}}$ at tree level is:

$m_{1 H}^{2}+m_{2 H}^{2} \geq 2\left|m_{12}^{2}\right|$ (required for stability of $V_{\text {Higgs }}$ ) $\left|m_{12}^{2}\right|^{2}>m_{1 H}^{2} m_{2 H}^{2}$ (required for $S U(2)_{\mathrm{L}} \times U(1)_{\mathrm{Y}}$ breaking).

We will proceed on the assumption that these conditions are satisfied.

In the following we consider the mass eigenstates of the MSSM. Any set of particles of a given spin, baryon number, lepton number and the same $S U(3)_{\mathrm{c}} \times U(1)_{\mathrm{em}}$ quantum numbers can mix. Therefore, in principle, there can be mixing in all the sectors of the MSSM and one must diagonalize mass matrices to obtain the mass eigenstates and the corresponding eigenvalues $[2,5,24]$. We consider here all the sectors, except the Higgs sector that we prefer to analyze elsewhere [21].

\subsection{Squarks}

One must diagonalize $6 \times 6$ matrices corresponding to the weak eigenstate basis $\left\{\tilde{q}_{i_{L}}, \tilde{q}_{i_{R}}\right\}$ where $i=1,2,3$ are the generation labels. We will ignore mixing between sfermions of different generations to avoid unacceptable large flavor changing neutral currents and give here the $2 \times 2$ matrix corresponding to the one generation case. As above, we choose to use the notation of the third family. The mixing mass matrices for the stop and sbottom squarks in the $\left(\tilde{t}_{L}, \tilde{t}_{R}\right)$ and $\left(\tilde{b}_{L}, \tilde{b}_{R}\right)$ bases respectively are as follows:

$$
\hat{M}_{\tilde{t}}^{2}=\left(\begin{array}{cc}
L & \varepsilon \\
\varepsilon & R .
\end{array}\right), \quad \hat{M}_{\tilde{b}}^{2}=\left(\begin{array}{cc}
L^{\prime} & \varepsilon^{\prime} \\
\varepsilon^{\prime} & R^{\prime}
\end{array}\right),
$$

Notice that these are generic matrices with, in principle, unconstrained and unrelated matrix elements. The only constraint comes from $S U(2)_{\mathrm{L}} \times U(1)_{\mathrm{Y}}$ gauge invariance which imposes the equality of the explicit breaking $\tilde{t}_{L}^{*} \tilde{t}_{L}$ and $\tilde{b}_{L}^{*} \tilde{b}_{L}$ mass terms. Thus, if the SUSY breaking is just the explicit one, one has $L=L^{\prime}$. This equality can be distorted by mass terms whose origin is not explicit SUSY breaking but spontaneous breaking of the $S U(2)_{\mathrm{L}} \times U(1)_{\mathrm{Y}}$ symmetry. In the large SUSY masses limit that we are interested in, $L$ and $L^{\prime}$ being of the order of $(1 \mathrm{TeV})^{2}$, this distortion is relatively small since it goes as $\left(m_{t}^{2}-m_{b}^{2}\right)$ and/or it is proportional to $m_{z}^{2}$.

We give here their specific expressions for completeness but we will keep the generic form, (6), through most of this paper:

$$
\begin{aligned}
L & =M_{\tilde{Q}}^{2}+m_{t}^{2}+m_{z}^{2}\left(\frac{1}{2}-Q_{t} s_{W}^{2}\right) \cos 2 \beta, \\
R & =M_{\tilde{U}}^{2}+m_{t}^{2}+m_{Z}^{2} Q_{t} s_{W}^{2} \cos 2 \beta, \\
\varepsilon & =m_{t}\left(A_{t}-\mu \cot \beta\right), \\
L^{\prime} & =M_{\tilde{Q}}^{2}+m_{b}^{2}-m_{Z}^{2}\left(\frac{1}{2}+Q_{b} s_{W}^{2}\right) \cos 2 \beta, \\
R^{\prime} & =M_{\tilde{D}}^{2}+m_{b}^{2}+m_{z}^{2} Q_{b} s_{W}^{2} \cos 2 \beta, \\
\varepsilon^{\prime} & =m_{b}\left(A_{b}-\mu \tan \beta\right),
\end{aligned}
$$

where $Q_{t}=2 / 3, Q_{b}=-1 / 3, s_{W}^{2} \equiv \sin ^{2} \theta_{W}$ and $\tan \beta \equiv$ $v_{2} / v_{1}$.

The mass eigenstates are denoted by $\tilde{t}_{1}, \tilde{t}_{2}, \tilde{b}_{1}, \tilde{b}_{2}$ and are related to the weak eigenstates $\tilde{t}_{L}, \tilde{t}_{R}, \tilde{b}_{L}, \tilde{b}_{R}$ by orthogonal matrices:

$$
\begin{aligned}
& \left(\begin{array}{c}
\tilde{t}_{L} \\
\tilde{t}_{R}
\end{array}\right)=R_{t}\left(\begin{array}{c}
\tilde{t}_{1} \\
\tilde{t}_{2}
\end{array}\right), \quad\left(\begin{array}{l}
\tilde{b}_{L} \\
\tilde{b}_{R}
\end{array}\right)=R_{b}\left(\begin{array}{l}
\tilde{b}_{1} \\
\tilde{b}_{2}
\end{array}\right) . \\
& R_{t}=\left(\begin{array}{cc}
c_{t} & -s_{t} \\
s_{t} & c_{t}
\end{array}\right), \quad R_{b}=\left(\begin{array}{cc}
c_{b} & -s_{b} \\
s_{b} & c_{b}
\end{array}\right) .
\end{aligned}
$$

where $c_{q} \equiv \cos \phi_{q}, s_{q} \equiv \sin \phi_{q}, q=t, b$.

The corresponding squared-mass eigenvalues and the mixing angles are given in terms of the generic matrix elements as follows:

$$
\begin{aligned}
& \tilde{m}_{t_{1,2}}^{2}=\frac{1}{2}(L+R) \pm \frac{1}{2}\left[(L-R)^{2}+4 \varepsilon^{2}\right]^{\frac{1}{2}}, \\
& \tilde{m}_{b_{1,2}}^{2}=\frac{1}{2}\left(L^{\prime}+R^{\prime}\right) \pm \frac{1}{2}\left[\left(L^{\prime}-R^{\prime}\right)^{2}+4 \varepsilon^{\prime 2}\right]^{\frac{1}{2}}, \\
& \tan 2 \phi_{t}=\frac{2 \varepsilon}{L-R} ; \quad \tan 2 \phi_{b}=\frac{2 \varepsilon^{\prime}}{L^{\prime}-R^{\prime}} .
\end{aligned}
$$

\subsection{Sleptons}

Similar formulae hold for the sleptons sector but replacing $\tilde{t}_{L} \rightarrow \tilde{\nu}, \tilde{t}_{R} \rightarrow 0, \tilde{b}_{L} \rightarrow \tilde{\tau}_{L}, \tilde{b}_{R} \rightarrow \tilde{\tau}_{R}$ and the corresponding changes in the rotation matrices $R_{\nu}$ and $R_{\tau}$ which relate the weak eigenstates $(\tilde{\nu}),\left(\tilde{\tau}_{L}, \tilde{\tau}_{R}\right)$ with the mass eigenstates $(\tilde{\nu}),\left(\tilde{\tau}_{1}, \tilde{\tau}_{2}\right)$, respectively:

$$
R_{\nu}=\left(\begin{array}{ll}
1 & 0 \\
0 & 1
\end{array}\right), \quad R_{\tau}=\left(\begin{array}{cc}
c_{\tau} & -s_{\tau} \\
s_{\tau} & c_{\tau}
\end{array}\right)
$$


The mass squared eigenvalues are $\tilde{m}_{\nu}^{2}, \tilde{m}_{\tau_{1}}^{2}, \tilde{m}_{\tau_{2}}^{2}$ respectively. The specific formulae (7) for the mass matrix elements must also be correspondingly replaced:

$$
\begin{aligned}
& L \rightarrow M_{\tilde{L}}^{2}+\frac{1}{2} m_{Z}^{2} \cos 2 \beta, \quad R \rightarrow 0, \quad \varepsilon \rightarrow 0, \\
& L^{\prime} \rightarrow M_{\tilde{L}}^{2}+m_{\tau}^{2}-m_{Z}^{2}\left(\frac{1}{2}-s_{W}^{2}\right) \cos 2 \beta, \\
& R^{\prime} \rightarrow M_{\tilde{E}}^{2}+m_{\tau}^{2}-m_{Z}^{2} s_{W}^{2} \cos 2 \beta, \\
& \varepsilon^{\prime} \rightarrow m_{\tau}\left(A_{\tau}-\mu \tan \beta\right) .
\end{aligned}
$$

From the above formulae (7) and (13), we see that the $\tilde{f}_{L}-\tilde{f}_{R}$ mixing is unimportant for most of the sfermions except for the stop. In the case $\tan \beta \gg 1$, the mixing in the sbottom sector may also be non-negligible.

\subsection{Charginos}

The charged gauginos, $\tilde{W}^{ \pm}$, and the charged Higgsinos, $\tilde{H}^{ \pm}$, can mix; the resulting mass eigenstates are the charginos. The 4-component Dirac fermions that represent these two charginos are denoted here by $\tilde{\chi}_{1}^{+}$and $\tilde{\chi}_{2}^{+}$. The mass matrix in the $\left(\tilde{W}^{+}, \tilde{H}^{+}\right)$basis can be written generically as:

$$
X=\left(\begin{array}{cc}
M_{2} & \varepsilon_{1} \\
\varepsilon_{2} & \mu
\end{array}\right) .
$$

As in the case of squarks and sleptons there are three types of contributions. There are contributions to the diagonal elements that come from explicit SUSY breaking, namely the Majorana mass $M_{2}$, and contributions that preserve SUSY, namely, the $\mu$-term. Both diagonal terms preserve $S U(2)_{\mathrm{L}} \times U(1)_{\mathrm{Y}}$ invariance. The offdiagonal terms come from the spontaneous breaking of the $S U(2)_{\mathrm{L}} \times U(1)_{\mathrm{Y}}$ symmetry. More specifically, when the Higgs field in the SUSY invariant interaction terms with a gaugino and a Higgsino is replaced by its vev, it gives rise to the following mixing entries,

$$
\varepsilon_{1}=\sqrt{2} m_{W} \sin \beta, \quad \varepsilon_{2}=\sqrt{2} m_{W} \cos \beta .
$$

Notice that $X$ is not symmetric unless $\tan \beta=1$. Therefore, two different unitary $2 \times 2$ matrices $U$ and $W$ are required to diagonalize the chargino mass matrix:

$$
\tilde{M}^{+}=\operatorname{diag}\left(\tilde{M}_{1}^{+}, \tilde{M}_{2}^{+}\right)=U^{*} X W^{-1} .
$$

In principle, the diagonal elements can be either positive or negative. We choose $M_{2}$ to be positive and $\mu$ can be either positive or negative. The physical chargino masses, $\left|\tilde{M}_{1}^{+}\right|,\left|\tilde{M}_{2}^{+}\right|$, are defined to be positive.

In case of negative eigenvalues we follow the procedure of the second paper in [2]. We define a new matrix $V$ such that,

$$
U^{*} X V^{-1}=\operatorname{diag}\left(\left|\tilde{M}_{1}^{+}\right|,\left|\tilde{M}_{2}^{+}\right|\right),
$$

and express all the interactions in terms of $U$ and $V$. $V$ can be trivially obtained from $W$ by changing all the signs in the file corresponding to the negative eigenvalue, i.e,
$V_{k l}=\eta_{k} W_{k l}$ (no sum over $k$ ) where $\eta_{k}$ is the sign of the eigenvalue $\tilde{M}_{k}^{+}(k, l=1,2)$.

The corresponding squared mass eigenvalues are:

$$
\begin{aligned}
\tilde{M}_{1,2}^{+2}= & \frac{1}{2}\left(M_{2}^{2}+\mu^{2}+2 m_{W}^{2}\right) \\
& \pm \frac{1}{2}\left[\left(M_{2}+\mu\right)^{2}\left[\left(M_{2}-\mu\right)^{2}+4 m_{W}^{2}\right]\right]^{\frac{1}{2}}
\end{aligned}
$$

Notice that to reach the large SUSY masses limit that we are interested in, it is necessary to consider the mass parameters in the range $M_{2}, \mu \gg m_{W}$ and therefore, to a very good approximation, the off diagonal elements of $X$ in (14) are negligible as compared to the diagonal elements. The mixing is small and $\tilde{\chi}_{1}^{+}$will be predominantly gaugino with a mass close to $M_{2}$, whereas $\tilde{\chi}_{2}^{+}$will be predominantly Higgsino with a mass close to $|\mu|$. In this case,

$$
U=W=\left(\begin{array}{ll}
1 & 0 \\
0 & 1
\end{array}\right),
$$

and if $\mu \geq 0$ or $\mu<0 V$ will be respectively:

$$
V=\left(\begin{array}{ll}
1 & 0 \\
0 & 1
\end{array}\right), \quad \text { or } \quad V=\left(\begin{array}{cc}
1 & 0 \\
0 & -1
\end{array}\right) \text {. }
$$

The corrections to these matrices are suppressed by powers of $\left(m_{W} / M\right)$ where $M$ is the largest of $M_{2}$ and $\mu$.

\subsection{Neutralinos}

The neutral gauginos $\tilde{B}$ and $\tilde{W}_{3}$ and the neutral Higgsinos $\tilde{H}_{1}^{0}, \tilde{H}_{2}^{0}$ can mix; the resulting mass eigenstates are the neutralinos. The 4-component Majorana fermions which represent these 4 neutralinos are denoted here by $\tilde{\chi}_{1}^{0}, \tilde{\chi}_{2}^{0}, \tilde{\chi}_{3}^{0}$ and $\tilde{\chi}_{4}^{0}$, following the standard notation given in $[2]$.

The mass matrix in the $\left(\tilde{B}, \tilde{W}_{3}, \tilde{H}_{1}^{0}, \tilde{H}_{2}^{0}\right)$ basis can be written generically as:

$$
Y=\left(\begin{array}{cccc}
M_{1} & 0 & \varepsilon_{1}^{\prime} & \varepsilon_{2}^{\prime} \\
0 & M_{2} & \varepsilon_{3}^{\prime} & \varepsilon_{4}^{\prime} \\
\varepsilon_{1}^{\prime} & \varepsilon_{3}^{\prime} & 0 & -\mu \\
\varepsilon_{2}^{\prime} & \varepsilon_{4}^{\prime} & -\mu & 0
\end{array}\right),
$$

Similarly to the chargino case, there are the explicit SUSY breaking mass terms in the diagonal, $M_{1}$ and $M_{2}$, and the SUSY preserving $\mu$-mass terms connecting $\tilde{H}_{1}^{0}$ and $\tilde{H}_{2}^{0}$. The rest of the off-diagonal terms come from the spontaneous breaking of $S U(2)_{\mathrm{L}} \times U(1)_{\mathrm{Y}}$ and are specifically given by:

$$
\begin{aligned}
& \varepsilon_{1}^{\prime}=-m_{Z} \cos \beta \sin \theta_{W}, \quad \varepsilon_{2}^{\prime}=m_{Z} \sin \beta \sin \theta_{W} \\
& \varepsilon_{3}^{\prime}=m_{Z} \cos \beta \cos \theta_{W}, \quad \varepsilon_{4}^{\prime}=-m_{Z} \sin \beta \cos \theta_{W} .
\end{aligned}
$$

Since $Y$ is symmetric, only one $4 \times 4$ unitary matrix, $Z$, is required to diagonalize it,

$$
\tilde{M}^{0}=\operatorname{diag}\left(\tilde{M}_{1}^{0}, \tilde{M}_{2}^{0}, \tilde{M}_{3}^{0}, \tilde{M}_{4}^{0}\right)=Z^{*} Y Z^{-1} .
$$


Here the diagonal elements can be either positive or negative. As in the chargino case, we choose the Majorana masses $M_{1}, M_{2}$ to be positive and allow $\mu$ to be positive or negative. The physical neutralino masses $\left|\tilde{M}_{1}^{0}\right|,\left|\tilde{M}_{2}^{0}\right|$, $\left|\tilde{M}_{3}^{0}\right|,\left|\tilde{M}_{4}^{0}\right|$ are defined to be positive. In case of negative eigenvalues, one defines a new matrix $N$ such that,

$$
N^{*} Y N^{-1}=\operatorname{diag}\left(\left|\tilde{M}_{1}^{0}\right|,\left|\tilde{M}_{2}^{0}\right|,\left|\tilde{M}_{3}^{0}\right|,\left|\tilde{M}_{4}^{0}\right|\right),
$$

and express all the interactions in terms of $N . N$ can be trivially obtained from $Z$ by multiplying all the entries in the file corresponding to the negative eigenvalue by the imaginary unity; i.e, $N_{k l}=\eta_{k}^{\prime} Z_{k l}$ (no sum over k), where $\eta_{k}^{\prime}=1$ if the eigenvalue $\tilde{M}_{k}^{0}$ is positive and $\eta_{k}^{\prime}=i$ if the eigenvalue $\tilde{M}_{k}^{0}$ is negative $(k, l=1,2,3,4)$.

In the large SUSY masses limit important simplifications do occur. In order to get the four neutralino masses larger than the electroweak scale it is necessary to consider the mass parameters in the range $M_{1}, M_{2}, \mu \gg m_{Z}$. Therefore, to a very good approximation, the off-diagonal terms $\varepsilon_{i}^{\prime}(i=1,2,3,4)$ are negligible as compared to $M_{1}, M_{2}$ and $\mu$. The physical mass eigenstates $\tilde{\chi}_{i}^{0},(i=1, \ldots, 4)$ are predominantly $\tilde{B}, \tilde{W}_{3},\left(\tilde{H}_{1}^{0}+\tilde{H}_{2}^{0}\right) / \sqrt{2}$ and $\left(\tilde{H}_{1}^{0}-\tilde{H}_{2}^{0}\right) / \sqrt{2}$, and their corresponding masses are close to $M_{1}, M_{2},|\mu|$ and $|\mu|$ respectively.

In this case,

$$
Z=\left(\begin{array}{cccc}
1 & 0 & 0 & 0 \\
0 & 1 & 0 & 0 \\
0 & 0 & \frac{1}{\sqrt{2}} & \frac{1}{\sqrt{2}} \\
0 & 0 & \frac{1}{\sqrt{2}} & -\frac{1}{\sqrt{2}}
\end{array}\right)
$$

and if $\mu \geq 0$ or $\mu<0, \mathrm{~N}$ will be respectively:

$$
\begin{aligned}
N & =\left(\begin{array}{cccc}
1 & 0 & 0 & 0 \\
0 & 1 & 0 & 0 \\
0 & 0 & \frac{i}{\sqrt{2}} & \frac{i}{\sqrt{2}} \\
0 & 0 & \frac{1}{\sqrt{2}} & -\frac{1}{\sqrt{2}}
\end{array}\right), \text { or } \\
N & =\left(\begin{array}{cccc}
1 & 0 & 0 & 0 \\
0 & 1 & 0 & 0 \\
0 & 0 & \frac{1}{\sqrt{2}} & \frac{1}{\sqrt{2}} \\
0 & 0 & \frac{i}{\sqrt{2}} & -\frac{i}{\sqrt{2}}
\end{array}\right) .
\end{aligned}
$$

\subsection{The relevant Lagrangian and notation}

Once the mass eigenstates that we plan to the integrate out have been specified, we need to specify in addition the relevant interaction terms of the supersymmetric Lagrangian $\mathcal{L}_{\text {SUSY }}$. Since, in the present paper, we aim to calculate the one-loop effective action for external $W^{ \pm}, Z$ and $\gamma$ gauge bosons, it is easy to see that the relevant interaction terms are those that connect gauge bosons with sfermions on one hand, and gauge bosons with neutralinos and charginos on the other hand. The terms connecting inos with sfermions are not relevant at one loop level. We write these interaction terms as well as the free Lagrangian in the mass-eigenstate basis which is the one that will be used in the rest of the paper. All together the relevant terms for the two points functions are the following:

$$
\mathcal{L}_{\operatorname{MSSM}}\left(V, \tilde{f}, \tilde{\chi}^{+}, \tilde{\chi}^{o}\right)=\mathcal{L}^{(0)}(V)+\mathcal{L}_{\tilde{f}}(V, \tilde{f})+\mathcal{L}_{\tilde{\chi}}(V, \tilde{\chi}),
$$

where $\mathcal{L}^{(0)}(V)$ is the standard quadratic Lagrangian in the $R_{\xi}$ gauge for the electroweak gauge bosons $V=W^{ \pm}, Z, \gamma$, and $\mathcal{L}_{\tilde{f}}(V, \tilde{f})$ and $\mathcal{L}_{\tilde{\chi}}(V, \tilde{\chi})$ are the Lagrangians for the sfermions and the neutralinos and charginos respectively. We use here a compact notation that is convenient for the integration in the path integral formalism. $\mathcal{L}_{\tilde{f}}$ is defined as follows:

$$
\mathcal{L}_{\tilde{f}}(V, \tilde{f})=\mathcal{L}_{\tilde{f}}^{(0)}(\tilde{f})+\mathcal{L}_{\tilde{f}}^{(1)}(V, \tilde{f})+\mathcal{L}_{\tilde{f}}^{(2)}(V, \tilde{f}),
$$

where $\mathcal{L}_{\tilde{f}}^{(0)}(\tilde{f})$ is the sfermions free Lagrangian:

$$
\mathcal{L}_{\tilde{f}}^{(0)}(\tilde{f})=\sum_{\tilde{f}}\left[\left(\partial_{\mu} \tilde{f}^{+} \partial^{\mu} \tilde{f}\right)-\left(\tilde{f}^{+} \tilde{M}_{f}^{2} \tilde{f}\right)\right]
$$

and $\tilde{f}$ is the shorthand notation for sfermions of all types. It must be understood as a column matrix with four entries, containing either the four mass eigenstates if it refers to squarks or the three mass eigenstates if it refers to sleptons. If we use the third generation notation, as before, it reads:

$$
\tilde{f} \equiv\left(\begin{array}{c}
\tilde{t}_{1} \\
\tilde{t}_{2} \\
\tilde{b}_{1} \\
\tilde{b}_{2}
\end{array}\right) \quad \text { if } \quad \tilde{f}=\tilde{q} \quad ; \quad \tilde{f} \equiv\left(\begin{array}{c}
\tilde{\nu} \\
0 \\
\tilde{\tau}_{1} \\
\tilde{\tau}_{2}
\end{array}\right) \quad \text { if } \tilde{f}=\tilde{l}
$$

the sum $\sum_{\tilde{f}}$ is over the three generations and, in the case of squarks, it runs also over the $N_{c}$ color indexes. The corresponding squared mass matrices are:

$$
\begin{array}{lrl}
\tilde{M}_{f}^{2}=\operatorname{diag}\left(\tilde{m}_{t_{1}}^{2}, \tilde{m}_{t_{2}}^{2}, \tilde{m}_{b_{1}}^{2}, \tilde{m}_{b_{2}}^{2}\right) \quad \text { if } & \tilde{f}=\tilde{q} ; \\
\tilde{M}_{f}^{2}=\operatorname{diag}\left(\tilde{m}_{\nu}^{2}, 0, \tilde{m}_{\tau_{1}}^{2}, \tilde{m}_{\tau_{2}}^{2}\right) \quad \text { if } & & \tilde{f}=\tilde{l} .
\end{array}
$$

The interaction Lagrangian of sfermions and gauge bosons consists of two parts. $\mathcal{L}_{\tilde{f}}^{(1)}$ gives the interactions of two sfermions and one gauge boson and $\mathcal{L}_{\tilde{f}}^{(2)}$ gives the interactions of two sfermions and two gauge bosons:

$$
\begin{gathered}
\mathcal{L}_{\tilde{f}}^{(1)}(V, \tilde{f})=\sum_{\tilde{f}}\left\{-i e A_{\mu} \tilde{f}^{+} \hat{Q}_{f} \overleftrightarrow{\partial^{\mu}} \tilde{f}-\frac{i g}{c_{W}} Z_{\mu} \tilde{f}^{+} \hat{G}_{f} \overleftrightarrow{\partial^{\mu}} \tilde{f}\right. \\
\left.-\frac{i g}{\sqrt{2}}\left[W_{\mu}^{+} \tilde{f}^{+} \Sigma_{f}^{t b} \overleftrightarrow{\partial^{\mu}} \tilde{f}+W_{\mu}^{-} \tilde{f}^{+} \Sigma_{f}^{b t} \overleftrightarrow{\partial^{\mu}} \tilde{f}\right]\right\}, \\
\mathcal{L}_{\tilde{f}}^{(2)}(V, \tilde{f})=\sum_{\tilde{f}}\left\{e^{2} A_{\mu} A^{\mu} \tilde{f}^{+} \hat{Q}_{f} \hat{Q}_{f} \tilde{f}\right. \\
+\frac{g^{2}}{c_{W}^{2}} Z_{\mu} Z^{\mu} \tilde{f}^{+} \hat{G}_{f} \hat{G}_{f} \tilde{f}+\frac{2 g e}{c_{W}} A_{\mu} Z^{\mu} \tilde{f}^{+} \hat{Q}_{f} \hat{G}_{f} \tilde{f}
\end{gathered}
$$




$$
\begin{aligned}
& +\frac{1}{2} g^{2} W_{\mu}^{+} W^{\mu-} \tilde{f}^{+} \Sigma_{f} \tilde{f}+\frac{e g}{\sqrt{2}} y_{f} A_{\mu} W^{\mu+} \tilde{f}^{+} \Sigma_{f}^{t b} \tilde{f} \\
& +\frac{e g}{\sqrt{2}} y_{f} A_{\mu} W^{\mu-} \tilde{f}^{+} \Sigma_{f}^{b t} \tilde{f}-\frac{g^{2}}{\sqrt{2}} y_{f} \frac{s_{W}^{2}}{c_{W}} Z_{\mu} W^{\mu_{+}} \tilde{f}^{+} \Sigma_{f}^{t b} \tilde{f} \\
& \left.-\frac{g^{2}}{\sqrt{2}} y_{f} \frac{s_{W}^{2}}{c_{W}} Z_{\mu} W^{\mu-} \tilde{f}^{+} \Sigma_{f}^{b t} \tilde{f}\right\},
\end{aligned}
$$

where:

$$
\begin{aligned}
\hat{Q}_{f} & =R_{f}^{-1} Q_{f} R_{f}, \hat{G}_{f}=R_{f}^{-1} G_{f} R_{f} \\
Q_{f} & =\operatorname{diag}\left(Q_{t}, Q_{t}, Q_{b}, Q_{b}\right) \\
G_{f} & =\operatorname{diag}\left(\frac{1}{2}-Q_{t} s_{W}^{2},-Q_{t} s_{W}^{2},-\frac{1}{2} Q_{b} s_{W}^{2},-Q_{b} s_{W}^{2}\right) \\
y_{f} & =\frac{1}{3} \quad \text { if } \quad \tilde{f}=\tilde{q} ; y_{f}=-1 \quad \text { if } \tilde{f}=\tilde{l} \\
\Sigma_{f}^{t b} & =\left(\begin{array}{cc}
0 & \hat{\sigma}_{t b} \\
0 & 0
\end{array}\right)=\left(\Sigma_{f}^{b t}\right)^{T}, \quad \hat{\sigma}_{t b}=R_{t}^{-1} \sigma R_{b} \\
\Sigma_{f} & =\left(\begin{array}{cc}
\hat{\sigma_{t}} & 0 \\
0 & \hat{\sigma_{b}}
\end{array}\right), \hat{\sigma}_{t, b}=R_{t, b}^{-1} \sigma R_{t, b} \\
\sigma & =\left(\begin{array}{cc}
1 & 0 \\
0 & 0
\end{array}\right), R_{f}=\left(\begin{array}{cc}
R_{t} & 0 \\
0 & R_{b}
\end{array}\right)
\end{aligned}
$$

and $R_{t}, R_{b}$ have been defined in (9).

The above expressions have been written for $\tilde{f}=\tilde{q}$. For $\tilde{f}=\tilde{l}$ analogous expressions are obtained by changing $t \rightarrow \nu, b \rightarrow \tau$, and the corresponding changes in the couplings and rotation matrices. For brevity, we will use in the following the squarks notation.

The Lagrangian for neutralinos and charginos is:

$$
\mathcal{L}_{\tilde{\chi}}(V, \tilde{\chi})=\mathcal{L}_{\tilde{\chi}}^{(o)}(\tilde{\chi})+\mathcal{L}_{\tilde{\chi}}^{(1)}(V, \tilde{\chi})
$$

where $\mathcal{L}_{\tilde{\chi}}^{(o)}$ is the free Lagrangian:

$$
\mathcal{L}_{\tilde{\chi}}^{(0)}(\tilde{\chi})=\frac{1}{2} \overline{\tilde{\chi}}^{o}\left(i \not \partial-\tilde{M}^{o}\right) \tilde{\chi}^{o}+\overline{\tilde{\chi}}^{+}\left(i \not \partial-\tilde{M}^{+}\right) \tilde{\chi}^{+}
$$

with,

$$
\tilde{\chi}^{o}=\left(\begin{array}{c}
\tilde{\chi}_{1}^{o} \\
\tilde{\chi}_{2}^{o} \\
\tilde{\chi}_{3}^{o} \\
\tilde{\chi}_{4}^{o}
\end{array}\right) \quad ; \quad \tilde{\chi}^{+}=\left(\begin{array}{c}
\tilde{\chi}_{1}^{+} \\
\tilde{\chi}_{2}^{+}
\end{array}\right)
$$

and the mass matrices $\tilde{M}^{+}$and $\tilde{M}^{o}$ have been defined in (16) and (23) respectively.

The interaction Lagrangian $\mathcal{L}_{\tilde{\chi}}^{(1)}$ consists of three parts,

$$
\begin{aligned}
\mathcal{L}_{\tilde{\chi}}^{(1)}(V, \tilde{\chi})= & \mathcal{L}_{o}^{(1)}\left(V, \tilde{\chi}^{o}\right)+\mathcal{L}_{+}^{(1)}\left(V, \tilde{\chi}^{+}\right) \\
& +\mathcal{L}_{+o}^{(1)}\left(V, \tilde{\chi}^{+}, \tilde{\chi}^{o}\right),
\end{aligned}
$$

$\mathcal{L}_{o}^{(1)}$ gives the interactions between neutralinos and gauge bosons, $\mathcal{L}_{+}^{(1)}$ the interactions between charginos and gauge bosons and $\mathcal{L}_{+o}^{(1)}$ the interactions that connect charginos, neutralinos and gauge bosons. More explicitly,

$$
\mathcal{L}_{o}^{(1)}\left(V, \tilde{\chi}^{o}\right)=\frac{g}{2 c_{W}} Z_{\mu} \overline{\tilde{\chi}}^{o} \gamma^{\mu}\left(O^{\prime \prime}{ }_{L} P_{L}+O^{\prime \prime}{ }_{R} P_{R}\right) \tilde{\chi}^{o}
$$

$$
\begin{aligned}
\mathcal{L}_{+}^{(1)}\left(V, \tilde{\chi}^{+}\right)= & -e A_{\mu} \overline{\tilde{\chi}}^{+} \gamma^{\mu} \tilde{\chi}^{+} \\
& +\frac{g}{c_{W}} Z_{\mu} \overline{\tilde{\chi}}^{+} \gamma^{\mu}\left(O^{\prime}{ }_{L} P_{L}+O^{\prime}{ }_{R} P_{R}\right) \tilde{\chi}^{+} \\
\mathcal{L}_{+o}^{(1)}\left(V, \tilde{\chi}^{+}, \tilde{\chi}^{o}\right)= & g W_{\mu}^{-} \overline{\tilde{\chi}}^{o} \gamma^{\mu}\left(O_{L} P_{L}+O_{R} P_{R}\right) \tilde{\chi}^{+} \\
& +g W_{\mu}^{+} \overline{\tilde{\chi}}^{+} \gamma^{\mu}\left(O_{L}^{+} P_{L}+O_{R}^{+} P_{R}\right) \tilde{\chi}^{o}
\end{aligned}
$$

where $O_{L, R}, O^{\prime}{ }_{L, R}$ and $O^{\prime \prime}{ }_{L, R}$ are the following $4 \times 2,2 \times 2$ and $4 \times 4$ matrices, respectively:

$$
\begin{aligned}
& \left(O_{L}\right)_{i j}=-\frac{1}{\sqrt{2}} N_{i 4} V_{j 2}^{*}+N_{i 2} V_{j 1}^{*} \\
& \left(O_{R}\right)_{i j}=\frac{1}{\sqrt{2}} N_{i 3}^{*} U_{j 2}+N_{i 2}^{*} U_{j 1} ; i=1,2,3,4 ; j=1,2, \\
& \left(O^{\prime}{ }_{L}\right)_{i j}=-V_{i 1} V_{j 1}^{*}-\frac{1}{2} V_{i 2} V_{j 2}^{*}+\delta_{i j} s_{W}^{2} ; \\
& \left(O^{\prime}{ }_{R}\right)_{i j}=-U_{i 1}^{*} U_{j 1}-\frac{1}{2} U_{i 2}^{*} U_{j 2}+\delta_{i j} s_{W}^{2} ; \quad i, j=1,2, \\
& \left(O^{\prime \prime}{ }_{L}\right)_{i j}=-\frac{1}{2} N_{i 3} N_{j 3}^{*}+\frac{1}{2} N_{i 4} N_{j 4}^{*} \text {; } \\
& \left(O^{\prime \prime}{ }_{R}\right)_{i j}=-\left(O^{\prime \prime}{ }_{L}\right)_{i j}^{*} \quad ; \quad i, j=1,2,3,4 \text {. }
\end{aligned}
$$

In particular, in the limit of large neutralino and chargino masses and by using the limiting expressions of $U, V$ and $N$ given in (19), (20) and (26) respectively, we get the following values for the coupling matrices, which are valid for $\mu \geq 0(\mu<0)$ :

$$
\begin{aligned}
& O_{L}=O_{R}=\left(\begin{array}{cc}
0 & 0 \\
1 & 0 \\
0 & \frac{-i}{2}\left(\frac{1}{2}\right) \\
0 & \frac{1}{2}\left(\frac{-i}{2}\right)
\end{array}\right) \\
& O^{\prime}{ }_{L}=O^{\prime}{ }_{R}=\left(\begin{array}{cc}
s_{W}{ }^{2}-1 & 0 \\
0 & s_{W}{ }^{2}-\frac{1}{2}
\end{array}\right) ; \\
& O^{\prime \prime}{ }_{L}=O^{\prime \prime}{ }_{R}=\left(\begin{array}{cccc}
0 & 0 & 0 & 0 \\
0 & 0 & 0 & 0 \\
0 & 0 & 0 & \frac{-i}{2}\left(\frac{i}{2}\right) \\
0 & 0 & \frac{i}{2}\left(\frac{-i}{2}\right) & 0
\end{array}\right) \text {. }
\end{aligned}
$$

\section{The large sparticle masses limit}

In this section we describe the large sparticle masses limit. We consider the situation where all the sparticle masses are much larger than the electroweak scale and therefore much heavier than their corresponding standard partners. In particular this could be the case if the sparticle masses are well above $m_{Z}, m_{W}$ and $m_{t}$ but still below the few $\mathrm{TeV}$ upper bound that is imposed by the standard solution to the hierarchy problem. The SUSY masses are also considered much larger than any of the external momenta in the Green functions that are studied in this work. The reason for this choice is because we are interested in the low energy limit of the MSSM and, in particular, in looking for any possible non-decoupling effect of heavy SUSY particles in the low energy observables as for instance the high precision observables at LEP. 
Generically we writte $\tilde{m}_{i}^{2} \gg M_{E W}^{2}, q^{2}$, where $\tilde{m}_{i}$ denotes any of the physical sparticle masses, $M_{E W}$ any of the electroweak masses $\left(m_{Z}, m_{W}, m_{t}, \ldots\right)$ and $q$ denotes any of the external momenta. As for the analytical computation, whenever we refer to the large sparticle masses limit of a given one-loop Feynman integral, we mean the asymptotic limit $\tilde{m}_{i} \rightarrow \infty$ for all sparticle masses that are involved in that integral. However, we would like to emphasize that this asymptotic limit is not fully defined unless one specifies in addition the relative sizes of the involved masses. In other words, the result may depend, in general, on the particular way this asymptotic limit is taken. This can be seen in many examples of one-loop integrals. In order to illustrate this, let us consider, for example, the well known scalar two-point function $B_{0}\left(q^{2}, m_{1}, m_{2}\right)$, as defined for instance in [15]. The result of this function in dimensional regularization is:

$$
\begin{aligned}
B_{0}\left(q^{2}, m_{1}, m_{2}\right)= & \Delta_{\epsilon}-\frac{1}{2}\left(\log \frac{m_{1}^{2}}{\mu_{o}^{2}}+\log \frac{m_{2}^{2}}{\mu_{o}^{2}}\right)+1 \\
& -\left(\frac{m_{1}^{2}+m_{2}^{2}}{m_{1}^{2}-m_{2}^{2}}\right) \log \frac{m_{1}}{m_{2}}+F\left(q^{2}, m_{1}, m_{2}\right),
\end{aligned}
$$

where $q^{2}$ is the external momentum, $m_{1}$ and $m_{2}$ are the masses of the two internal propagators, $\Delta_{\epsilon}$ and $\mu_{o}$ are defined in Appendix B and the explicit expression for the function $F\left(q^{2}, m_{1}, m_{2}\right)$ can be found in [15].

Let us consider the large masses limit, $m_{1}, m_{2} \gg q^{2}$ of $B_{0}$ in the two following situations:

$m_{1}^{2}=m_{2}^{2} \equiv m^{2}, m^{2} \gg q^{2}($ case $A)$ and

$m_{1}^{2}=2 m_{2}^{2} \equiv 2 m^{2}, m^{2} \gg q^{2}($ case $B)$.

By explicit computation of $B_{0}\left(q^{2}, m_{1}, m_{2}\right)$ in this two different limits we get:

case $A$

$$
B_{0}=\Delta_{\epsilon}-\log \frac{m^{2}}{\mu_{o}^{2}}+\left[\frac{2}{3}\left(\frac{q^{2}}{4 m^{2}}\right)+O\left(\frac{q^{4}}{m^{4}}\right)\right]
$$

case $B$

$$
B_{0}=\Delta_{\epsilon}-\log \frac{m^{2}}{\mu_{o}^{2}}+1-2 \log 2+\left[O\left(\frac{q^{4}}{m^{4}}\right)\right]
$$

The quantities in square brackets represent the decoupling effects since they vanish in the asymptotic limit $m^{2} \rightarrow \infty$. The remaining terms contain all the nondecoupling effects of particles 1 and 2 and they are the object of our interest here since they do not vanish in the asymptotic limit $m^{2} \rightarrow \infty$. It is clear from the above results that the two cases lead to different non-decoupling effects.

For the present study of large sparticle masses limit, $\tilde{m}_{i}^{2} \gg M_{E W}^{2}, q^{2}$ we consider the two following different possibilities for the physical masses $(i \neq j)$ :

$$
\begin{array}{ll}
\text { case } A & \left|\tilde{m}_{i}^{2}-\tilde{m}_{j}^{2}\right| \ll\left|\tilde{m}_{i}^{2}+\tilde{m}_{j}^{2}\right|, \\
\text { case } B & O\left(\left|\tilde{m}_{i}^{2}-\tilde{m}_{j}^{2}\right|\right) \approx O\left(\left|\tilde{m}_{i}^{2}+\tilde{m}_{j}^{2}\right|\right) .
\end{array}
$$

In the first case, the asymptotic limit $\tilde{m}_{i, j}^{2} \rightarrow \infty$ is taken such that, $\left|\frac{\tilde{m}_{i}^{2}-\tilde{m}_{j}^{2}}{\tilde{m}_{i}^{2}+\tilde{m}_{j}^{2}}\right| \ll 1$ and therefore this mass ratio is a good parameter for the large mass expansion. The extreme situation of total degeneracy $\tilde{m}_{i}^{2}=\tilde{m}_{j}^{2}$ can be considered as a particular example belonging to this case $A$.

In case $B$ the asymptotic limit $\tilde{m}_{i, j}^{2} \rightarrow \infty$ is taken such that, $\left|\frac{\tilde{m}_{i}^{2}-\tilde{m}_{j}^{2}}{\tilde{m}_{i}^{2}+\tilde{m}_{j}^{2}}\right| \approx O(1)$ and therefore it is not the proper parameter for the large mass expansion. Physically this situation corresponds to consider both masses $\tilde{m}_{i}$ and $\tilde{m}_{j}$ different and large with their difference being also large. The previous considered case $B$ for $B_{0}$ is one example belonging to this situation.

In the following we study how these two cases can be accomplished in the MSSM for each one of the sparticle sectors that are considered in this work.

\subsection{Large mass limit in the inos sector}

Given the particular form of the mass matrices in the inos sector, (14) and (21), the large chargino masses limit, $\tilde{M}_{1,2}^{+2} \gg M_{E W}^{2}$, and the large neutralino masses limit, $\tilde{M}_{1,2,3,4}^{0^{2}} \gg M_{E W}^{2}$, can only be accomplished if the three involved SUSY mass parameters are taken large, namely, if $M_{1}^{2}, M_{2}^{2}, \mu^{2} \gg M_{E W}^{2}$. As we have already mentioned in the previous section, the physical masses are, in this limit:

$$
\begin{aligned}
& \tilde{M}_{1}^{+} \approx M_{2}, \tilde{M}_{2}^{+} \approx|\mu|, \\
& \tilde{M}_{1}^{0} \approx M_{1}, \tilde{M}_{2}^{0} \approx M_{2}, \tilde{M}_{3}^{0} \approx|\mu|, \tilde{M}_{4}^{0} \approx|\mu| .
\end{aligned}
$$

In consequence, the two situations $A$ and $B$ above can be accomplished in the inos sector by choosing the corresponding possibilities for the parameters, $M_{1}, M_{2}$ and $\mu$. For instance, $\left|\tilde{M}_{1}^{+^{2}}-\tilde{M}_{2}^{+^{2}}\right| \ll\left|\tilde{M}_{1}^{+^{2}}+\tilde{M}_{2}^{+^{2}}\right|$ can be accomplished if one chooses $\left|M_{2}^{2}-\mu^{2}\right| \ll\left|M_{2}^{2}+\mu^{2}\right|$. However, by inspection of the coupling matrices in the inos-sector, (41), we see that only the following pairings will occur in the one-loop integrals: $\left(\tilde{\chi}_{1}^{+}, \tilde{\chi}_{2}^{o}\right),\left(\tilde{\chi}_{2}^{+}, \tilde{\chi}_{3}^{o}\right),\left(\tilde{\chi}_{2}^{+}, \tilde{\chi}_{4}^{o}\right)$, $\left(\tilde{\chi}_{1}^{+}, \tilde{\chi}_{1}^{+}\right),\left(\tilde{\chi}_{2}^{+}, \tilde{\chi}_{2}^{+}\right)$and $\left(\tilde{\chi}_{3}^{o}, \tilde{\chi}_{4}^{o}\right)$. Therefore, in this sector the masses that need to be compared when performing the large mass limit always belong to case $A$.

\subsection{Large mass limit in the sfermions sector}

Here the situation is very different since the mixing may not be negligible and hence it may play a relevant role in taking the large mass limit. For definitess and for illustrative purposes, let us consider here the stop-sbottom sector. First of all, the requirement that all sparticles be heavier that their corresponding partners reads in this case $\tilde{m}_{t_{1,2}}^{2}>m_{t}^{2}$ and $\tilde{m}_{b_{1,2}}^{2}>m_{b}^{2}$ and imply the following conditions on the mass matrix parameters of (6) : $\varepsilon^{2}<$ $L R+m_{t}^{4}-m_{t}^{2}(L+R)$ and $\varepsilon^{\prime 2}<L^{\prime} R^{\prime}+m_{b}^{4}-m_{b}^{2}\left(L^{\prime}+R^{\prime}\right)$.

On the other hand, the wanted large stop and sbottom masses limit, $\tilde{m}_{t_{1,2}}^{2}, \tilde{m}_{b_{1,2}}^{2} \gg M_{E W}^{2}$ can only be accomplished if $L, R, L^{\prime}, R^{\prime} \gg M_{E W}^{2}$, and therefore the above conditions translate into the simpler ones: $\varepsilon^{2}<L R$ and $\varepsilon^{\prime 2}<L^{\prime} R^{\prime}$ respectively. This means that in taking the 
large masses limit the mixing can be large but not arbitrarily large since it is bounded from above by these conditions.

By using the definitions in the MSSM of (7), these inequalities can be expressed in terms of the soft SUSY breaking masses and the $\mu$ parameter as follows:

$$
\begin{aligned}
& m_{t}^{2}\left(A_{t}-\mu \cot \beta\right)^{2}<M_{\tilde{Q}}^{2} M_{\tilde{U}}^{2}, \\
& m_{b}^{2}\left(A_{b}-\mu \tan \beta\right)^{2}<M_{\tilde{Q}}^{2} M_{\tilde{D}}^{2}, \\
& M_{\tilde{Q}}^{2}, M_{\tilde{D}}^{2}, M_{\tilde{U}}^{2} \gg m_{t}^{2}, m_{z}^{2},
\end{aligned}
$$

where the later condition implies, in turn, the limiting values:

$$
\tilde{m}_{t_{1}}^{2} \approx M_{\tilde{Q}}^{2}, \tilde{m}_{t_{2}}^{2} \approx M_{\tilde{U}}^{2}, \tilde{m}_{b_{1}}^{2} \approx M_{\tilde{Q}}^{2}, \tilde{m}_{b_{2}}^{2} \approx M_{\tilde{D}}^{2} .
$$

In summary, in other to get large stop and sbottom masses one needs large values of the SUSY breaking masses $M_{\tilde{Q}}$, $M_{\tilde{U}}$ and $M_{\tilde{D}}$ and in order not to get a too large mixing, the trilinear couplings $A_{t}, A_{b}$ and the $\mu$ parameter must be constrained from above by the previous inequalities. In particular, $\mu$ cannot be arbitrarily large when the rest of the parameters are kept fixed, since the lightest mass eigenvalues of (10) can be driven to non-physical negative values.

Notice that the $\mu$ parameter enters in both the sfermions and the inos sector and therefore the large sparticle masses limit in both sectors are not independent. More specifically, the large masses limit in the ino sector which requires a large value of $\mu$, must respect in addition the above restriction on $\mu$. In practice, this can be implemented by several different choices of the SUSY parameters.

Let us study now how the previous cases $A$ and $B$ can be reached in the sfermions sector. We present the discussion in terms of the six generic mass matrix parameters $L, R, \varepsilon, L^{\prime}, R^{\prime}, \varepsilon^{\prime}$. Notice that it is just once these are specified in terms of the MSSM parameters that there are some correlations among them which are relevant in studing the large sparticle masses limit.

Let us consider first the simplest case, corresponding to vanishing mixing:

$$
\begin{aligned}
\varepsilon & =\varepsilon^{\prime}=0 \Rightarrow s_{t}=s_{b}=0, \text { and } \\
\tilde{m}_{t_{1}}^{2} & =L, \tilde{m}_{t_{2}}^{2}=R, \tilde{m}_{b_{1}}^{2}=L^{\prime}, \tilde{m}_{b_{2}}^{2}=R^{\prime} .
\end{aligned}
$$

The only possible pairings in this case are: $\left(\tilde{t}_{1}, \tilde{t}_{1}\right)$, $\left(\tilde{t}_{2}, \tilde{t}_{2}\right),\left(\tilde{b}_{1}, \tilde{b}_{1}\right),\left(\tilde{b}_{2}, \tilde{b}_{2}\right)$ and $\left(\tilde{t}_{1}, \tilde{b}_{1}\right)$. Therefore there are just two masses to be compared, namely, $\tilde{m}_{t_{1}}$ and $\tilde{m}_{b_{1}}$. Generically, both possibilities $A$ and $B$ could occur. However, in restricting us to the MSSM parameters of (7) it is clear that only possibility $A$ does remain, since $\left|L-L^{\prime}\right| \ll$ $\left|L+L^{\prime}\right|$ is always true in the large sparticle masses limit of the MSSM. Notice that this situation applies to all squarks of the first and second generation and to all sleptons.

Let us consider next the case of non-vanishing mixings $\varepsilon \neq 0, \varepsilon^{\prime} \neq 0$. Notice that it is in fact the most realistic situation in the stop-sbottom sector of the MSSM since, as we have already said, $|\varepsilon|$ and $\left|\varepsilon^{\prime}\right|$ grow linearly with $\mu$ and this must be taken large $\left(\mu^{2} \gg M_{E W}^{2}\right)$ to get heavy inos. Generically, therefore, in taking the large masses limit the mixings $\varepsilon$ and $\varepsilon^{\prime}$ should not be held fixed.
For non-vanishing mixings, all possible pairings do occur: $\left(\tilde{t}_{1}, \tilde{t}_{1}\right),\left(\tilde{t}_{2}, \tilde{t}_{2}\right),\left(\tilde{b}_{1}, \tilde{b}_{1}\right),\left(\tilde{b}_{2}, \tilde{b}_{2}\right)$ and $\left(\tilde{t}_{1}, \tilde{t}_{2}\right),\left(\tilde{t}_{1}, \tilde{b}_{1}\right)$, $\left(\tilde{t}_{1}, \tilde{b}_{2}\right),\left(\tilde{b}_{1}, \tilde{b}_{2}\right),\left(\tilde{b}_{1}, \tilde{t}_{2}\right),\left(\tilde{t}_{2}, \tilde{b}_{2}\right)$. Therefore, all the mass pairs need to be compared. For illustrative purposes let us analize here the two cases $A$ and $B$ in particular for the $\left(\tilde{t}_{1}, \tilde{t}_{2}\right)$ pair.

$$
\text { case } A \quad\left|\frac{\tilde{m}_{t_{1}}^{2}-\tilde{m}_{t_{2}}^{2}}{\tilde{m}_{t_{1}}^{2}+\tilde{m}_{t_{2}}^{2}}\right| \ll 1 .
$$

By using (10) this condition can be written as,

$$
\begin{gathered}
{\left[\left(\frac{L-R}{L+R}\right)^{2}+\left(\frac{2 \varepsilon}{L+R}\right)^{2}\right]^{\frac{1}{2}} \ll 1} \\
\mathbb{\sharp} \\
\left(\frac{L-R}{L+R}\right) \ll 1 \text { and }\left(\frac{2 \varepsilon}{L+R}\right) \ll 1
\end{gathered}
$$

The proper parameter for the large mass expansion in terms of the physical masses of (50) is translated into the two small parameters of (51). Furthermore, within this case there are still two different possibilities:

A1) $L=R$

It can be solved exactly for all values of $\varepsilon \neq 0$ and $\varepsilon^{\prime} \neq 0$ and gives the limiting values,

$$
s_{t}=c_{t}=\frac{1}{\sqrt{2}} \Longrightarrow \tan 2 \phi_{t}=\frac{2 \varepsilon}{L-R} \rightarrow \infty .
$$

The proper parameter for the expansion in this case is $\left(\frac{2 \varepsilon}{L+R}\right)$.

\section{A2) $L \neq R$}

This is the most plausible situation in the MSSM. In this case the two above conditions in (51) can be written in terms of the MSSM parameters respectively as follows,

$$
\left|\frac{M_{\tilde{Q}}^{2}-M_{\tilde{U}}^{2}+O\left(M_{E W}^{2}\right)}{M_{\tilde{Q}}^{2}+M_{\tilde{U}}^{2}}\right| \ll 1
$$

and,

$$
\left|\frac{2 m_{t}\left(A_{t}-\mu \cot \beta\right)}{M_{\tilde{Q}}^{2}+M_{\tilde{U}}^{2}}\right| \ll 1
$$

Notice that if one assumes, as usual, that all the SUSY mass parameters are of the same order, namely, if $M_{\tilde{Q}}$, $M_{\tilde{U}}, A_{t}, \mu \sim O\left(M_{S U S Y}\right)$ with $M_{S U S Y} \gg M_{E W}$ being the effective SUSY breaking mass scale, then (53) is automatically fulfiled, since this small parameter goes as $O\left(\frac{M_{E W}}{M_{S U S Y}}\right)$. In order to get the first condition of (52) also automatically accomplished in the MSSM, one needs in addition to impose the equality of the two soft SUSY breaking parameters $M_{\tilde{Q}}=M_{\tilde{U}}$. In this case the small parameter of (52) goes as $O\left(\frac{M_{E W}^{2}}{M_{S U S Y}^{2}}\right)$. Any departure from this exact equality would lead us to a different situation which is considered next.

$$
\text { case } B \quad\left|\frac{\tilde{m}_{t_{1}}^{2}-\tilde{m}_{t_{2}}^{2}}{\tilde{m}_{t_{1}}^{2}+\tilde{m}_{t_{2}}^{2}}\right| \approx O(1) .
$$

This can be written as:

$$
\left[\left(\frac{L-R}{L+R}\right)^{2}+\left(\frac{2 \varepsilon}{L+R}\right)^{2}\right]^{\frac{1}{2}} \approx O(1)
$$


If, as before, one assumes $M_{\tilde{Q}}, M_{\tilde{U}}, A_{t}, \mu \sim O\left(M_{S U S Y}\right)$ then (55) can be traslated in terms of the MSSM parameters as:

$$
\left|\frac{M_{\tilde{Q}}^{2}-M_{\tilde{U}}^{2}}{M_{\tilde{Q}}^{2}+M_{\tilde{U}}^{2}}\right| \approx O(1),
$$

since (53) still holds.

This condition is naturally reached if one chooses, $\left(M_{\tilde{Q}}^{2}\right.$ $\left.-M_{\tilde{U}}^{2}\right) \sim O\left(M_{S U S Y}^{2}\right)$. The parameters in (54) and (56) are obviously not the proper parameters for the large mass expansion in this case, but the parameter $\left(\frac{2 \varepsilon}{L+R}\right)$ given in the MSSM by (53) is still a good one. Other proper choices are also available. In particular, both quantities, $\tan 2 \phi_{t}$ and $s_{t}$ turn out to be good parameters for the expansion in this case. In fact,

$$
\begin{aligned}
\tan 2 \phi_{t} & =\frac{2 \varepsilon}{L-R} \\
& \simeq \frac{2 m_{t}\left(A_{t}-\mu \cot \beta\right)}{M_{\tilde{Q}}^{2}-M_{\tilde{U}}^{2}} \approx O\left(\frac{M_{E W}}{M_{S U S Y}}\right),
\end{aligned}
$$

and similarly, $s_{t} \approx O\left(\frac{M_{E W}}{M_{S U S Y}}\right)$.

One can make a parallel discussion for the sbottom sector and conclude similarly that, $s_{b} \approx O\left(\frac{M_{E W}}{M_{S U S Y}}\right)$.

In consequence, case $B$ implies that only the following pairings do remain in the large squark masses limit: $\left(\tilde{t}_{1}, \tilde{t}_{1}\right),\left(\tilde{t}_{2}, \tilde{t}_{2}\right),\left(\tilde{b}_{1}, \tilde{b}_{1}\right),\left(\tilde{b}_{2}, \tilde{b}_{2}\right)$ and $\left(\tilde{t}_{1}, \tilde{b}_{1}\right)$ and therefore, once again, the masses to be compared are $\tilde{m}_{t_{1}}$ and $\tilde{m}_{b_{1}}$, which fulfil automatically the condition $\left|\tilde{m}_{t_{1}}^{2}-\tilde{m}_{b_{1}}^{2}\right| \ll$ $\left|\tilde{m}_{t_{1}}^{2}+\tilde{m}_{b_{1}}^{2}\right|$ in the MSSM.

In summary, the sfermion sector of the MSSM behaves in the large masses limit $\left(M_{S U S Y} \gg M_{E W}\right)$ as follows,

1. Sfermions $\tilde{f}_{1}, \tilde{f}_{2}$ other than stop and sbottom. The masses of their standard fermionic partners are neglected $\left(\varepsilon=\varepsilon^{\prime}=0\right)$ and,

$$
\left|\frac{\tilde{m}_{f_{1}}^{2}-\tilde{m}_{f_{2}}^{2}}{\tilde{m}_{f_{1}}^{2}+\tilde{m}_{f_{2}}^{2}}\right| \sim O\left(\frac{M_{E W}^{2}}{M_{S U S Y}^{2}}\right) \ll 1 .
$$

It is the proper parameter for the large mass expansion of a one loop integral involving the pairing $\left(\tilde{f}_{1}, \tilde{f}_{2}\right)$. The corresponding SUSY mass parameters $M_{\tilde{L}}, M_{\tilde{E}}, \ldots$ are just required to be of order $M_{S U S Y}$.

2. Stops and sbottoms $\left(\varepsilon \neq 0, \varepsilon^{\prime} \neq 0\right)$.

2a) If $M_{\tilde{Q}}=M_{\tilde{U}}=M_{\tilde{D}} \sim O\left(M_{S U S Y}\right)$ and $\mu, A_{t}, A_{b} \sim$ $O\left(M_{S U S Y}\right)$ then,

$$
\begin{aligned}
\left|\frac{\tilde{m}_{i}^{2}-\tilde{m}_{j}^{2}}{\tilde{m}_{i}^{2}+\tilde{m}_{j}^{2}}\right| \sim & O\left(\frac{M_{E W}^{2}}{M_{S U S Y}^{2}}\right) \ll 1, \\
& i, j=\tilde{t}_{1}, \tilde{t}_{2}, \tilde{b}_{1}, \tilde{b}_{2}(i \neq j)
\end{aligned}
$$

It is the proper parameter for the large mass expansion of a one-loop integral involving the pair $(i, j)$ with $i \neq j$. In the asymptotic limit $M_{S U S Y} \rightarrow \infty$ the mixing is maximal and $s_{t}, s_{b}$ tend to their limiting values, $s_{t}=s_{b}=\frac{1}{\sqrt{2}}$.

$2 b)$ If $M_{\tilde{Q}}, M_{\tilde{U}}, M_{\tilde{D}}, \mu, A_{t}, A_{b} \sim O\left(M_{S U S Y}\right)$ with $\left(M_{i}^{2}-M_{j}^{2}\right) \sim O\left(M_{S U S Y}^{2}\right), i, j=\tilde{Q}, \tilde{U}, \tilde{D}(i \neq j)$ then,

$$
s_{t}, s_{b} \sim O\left(\frac{M_{E W}}{M_{S U S Y}}\right) .
$$

In the asymptotic limit $M_{S U S Y} \rightarrow \infty$ then $s_{t}, s_{b} \rightarrow 0$ and all the one-loop integrals involving pairings $(i, j)$ with $i \neq j$ decouple except $\left(\tilde{t}_{1}, \tilde{b}_{1}\right)$ for which:

$$
\left|\frac{\tilde{m}_{t_{1}}^{2}-\tilde{m}_{b_{1}}^{2}}{\tilde{m}_{t_{1}}^{2}+\tilde{m}_{b_{1}}^{2}}\right| \sim O\left(\frac{M_{E W}^{2}}{M_{S U S Y}^{2}}\right) \ll 1 .
$$

In conclusion, the large sparticle masses limit in the MSSM can generically be studied in terms of the physical masses by considering the corresponding one-loop Feynman integrals in the limit $\tilde{m}_{i}^{2}, \tilde{m}_{j}^{2} \gg M_{E W}^{2}, q^{2}$ with either possibility $A$ :

$$
\left|\frac{\tilde{m}_{i}^{2}-\tilde{m}_{j}^{2}}{\tilde{m}_{i}^{2}+\tilde{m}_{j}^{2}}\right| \ll 1
$$

or possibility $B$ :

$$
\left|\frac{\tilde{m}_{i}^{2}-\tilde{m}_{j}^{2}}{\tilde{m}_{i}^{2}+\tilde{m}_{j}^{2}}\right| \approx O(1) \text { if } i \neq j .
$$

In this paper we have analized in full detail the possibility $A$ and we will demonstrate the decoupling of SUSY particles under this assumption. The different possibility $B$ is not analized generically in this work, but it will be studied for the particular case of $S, T$ and $U$ in Sect.6, where we will demonstrate that decoupling of sparticles does occur in both cases $A$ and $B$.

\section{Effective action for the electroweak gauge bosons in the MSSM}

Our aim is to compute the effective action for the standard particles, $\Gamma_{e f f}[\phi]$, that is defined through functional integration of all the sparticles of the MSSM. In short notation it is defined by,

$$
\mathrm{e}^{i \Gamma_{e f f}[\phi]}=\int[\mathrm{d} \tilde{\phi}] \mathrm{e}^{i \Gamma_{\mathrm{MSSM}}[\phi, \tilde{\phi}]}
$$

where $\phi=l, q, A, W^{ \pm}, Z, g, H$ are the SM particles; $\tilde{\phi}=$ $\tilde{l}, \tilde{q}, \tilde{A}, \tilde{W} \tilde{W}^{ \pm}, \tilde{Z}, \tilde{g}, \tilde{H}$ their supersymmetric partners, and

$$
\Gamma_{\mathrm{MSSM}}[\phi, \tilde{\phi}] \equiv \int d x \mathcal{L}_{\mathrm{MSSM}}(\phi, \tilde{\phi}) ; \mathrm{d} x \equiv \mathrm{d}^{4} x .
$$

In the present paper we are interested in the part of the effective action that contains the two point Green functions with external gauge bosons $\Gamma_{\mu \nu}^{V_{1} V_{2}}(k), V_{1}, V_{2}=$ $A, Z, W^{ \pm}$. This will allow us to study the decoupling properties of the gauge boson self-energies and from them we 
will deduce the corresponding analytical expressions for the well known parameters $S, T$ and $U$. The computation of the effective action will be performed at the one loop level by using dimensional regularization and will include the integration of all the sfermions $\tilde{f}$, the neutralinos $\tilde{\chi}^{o}$ and the charginos $\tilde{\chi}^{+}$. We leave the integration of the Higgs sector as well as the computation of other relevant parts of the effective action, as for instance the higher point Green functions or the Green functions with external fermions, for forthcoming works [21,22].

We start by defining the piece of the effective action that we want to compute,

$$
\begin{aligned}
& \mathrm{e}^{i \Gamma_{e f f}[V]} \\
& \quad=\int[\mathrm{d} \tilde{f}]\left[\mathrm{d} \tilde{f}^{*}\right]\left[\mathrm{d} \tilde{\chi}^{+}\right]\left[\mathrm{d} \overline{\tilde{\chi}}^{+}\right]\left[\mathrm{d} \tilde{\chi}^{o}\right] \mathrm{e}^{i \Gamma_{\mathrm{MSSM}}\left[V, \tilde{f}, \tilde{\chi}^{+}, \tilde{\chi}^{o}\right]}
\end{aligned}
$$

where,

$$
\begin{aligned}
\Gamma_{\mathrm{MSSM}}\left[V, \tilde{f}, \tilde{\chi}^{+}, \tilde{\chi}^{o}\right] \equiv & \int d x \mathcal{L}_{\mathrm{MSSM}}\left(V, \tilde{f}, \tilde{\chi}^{+}, \tilde{\chi}^{o}\right) \\
= & \int d x \mathcal{L}^{(0)}(V)+\int d x \mathcal{L}_{\tilde{f}}(V, \tilde{f}) \\
& +\int d x \mathcal{L}_{\tilde{\chi}}(V, \tilde{\chi}) \\
\equiv & \Gamma_{0}[V]+\Gamma_{\tilde{f}}[V, \tilde{f}]+\Gamma_{\tilde{\chi}}[V, \tilde{\chi}]
\end{aligned}
$$

and $\mathcal{L}_{\tilde{f}}, \mathcal{L}_{\tilde{\chi}}$ have been defined in (28) and (35) respectively.

In order to perform the functional integration, it is convenient to write the classical action in terms of operators. Thus by using (28) through (34) we get,

$$
\Gamma_{\tilde{f}}[V, \tilde{f}]=\left\langle\tilde{f}^{+} A_{\tilde{f}} \tilde{f}\right\rangle
$$

where,

$$
\begin{aligned}
A_{\tilde{f}} & \equiv A_{\tilde{f}}^{(0)}+A_{\tilde{f}}^{(1)}+A_{\tilde{f}}^{(2)} \\
\left\langle\tilde{f}^{+} A_{\tilde{f}}^{(i)} \tilde{f}\right\rangle & \equiv \sum_{\tilde{f}} \int d x d y \tilde{f}_{x}^{+} A_{\tilde{f} x y}^{(i)} \tilde{f}_{y}, i=0,1,2
\end{aligned}
$$

and the operators are:

$$
\begin{aligned}
A_{\tilde{f} x y}^{(0)} \equiv & \left(-\square-\tilde{M}_{f}^{2}\right)_{x} \delta_{x y} \\
A_{\tilde{f} x y}^{(1)} \equiv & -i e\left(\partial_{\mu} A^{\mu} \hat{Q}_{f}+2 \hat{Q}_{f} A_{\mu} \partial^{\mu}\right)_{x} \delta_{x y} \\
& -\frac{i g}{c_{w}}\left(\partial_{\mu} Z^{\mu} \hat{G}_{f}+2 \hat{G}_{f} Z_{\mu} \partial^{\mu}\right)_{x} \delta_{x y} \\
& -\frac{i g}{\sqrt{2}}\left(\partial_{\mu} W^{+\mu} \Sigma_{f}^{t b}+2 \Sigma_{f}^{t b} W_{\mu}^{+} \partial^{\mu}\right)_{x} \delta_{x y}+\text { h.c. } \\
A_{\tilde{f} x y}^{(2)} \equiv & \left(e^{2} \hat{Q}_{f}^{2} A_{\mu} A^{\mu}+\frac{2 g e}{c_{w}} A_{\mu} Z^{\mu} \hat{Q}_{f} \hat{G}_{f}+\frac{g^{2}}{c_{w}^{2}} \hat{G}_{f}^{2} Z_{\mu} Z^{\mu}\right. \\
& +\frac{1}{2} g^{2} \Sigma_{f} W_{\mu}^{+} W^{\mu-}+\frac{e g}{\sqrt{2}} y_{f} A_{\mu} W^{\mu+} \Sigma_{f}^{t b} \\
& +\frac{e g}{\sqrt{2}} y_{f} A_{\mu} W^{\mu-} \Sigma_{f}^{b t}-\frac{g^{2}}{\sqrt{2}} y_{f} \frac{s_{W}^{2}}{c_{W}} Z_{\mu} W^{\mu+} \Sigma_{f}^{t b} \\
& \left.-\frac{g^{2}}{\sqrt{2}} y_{f} \frac{s_{W}^{2}}{c_{W}} Z_{\mu} W^{\mu} \Sigma_{f}^{b t}\right)_{x} \delta_{x y} \cdot
\end{aligned}
$$

In these formulae and from now on we will use the compact notation,

$$
\begin{aligned}
& \phi(x) \equiv \phi_{x}, \delta(x-y) \equiv \delta_{x y}, A(x, y) \equiv A_{x y} \\
& \operatorname{Tr} A=\operatorname{tr} \int d x A_{x x}=\sum_{a} \int d x A_{x x}^{a a} .
\end{aligned}
$$

Analogously, by using (35) through (39) we get,

$$
\begin{aligned}
\Gamma_{\tilde{\chi}}[V, \tilde{\chi}]= & \frac{1}{2}\left\langle\overline{\tilde{\chi}}^{0}\left(A_{0}^{(0)}+A_{0}^{(1)}\right) \tilde{\chi}^{o}\right\rangle \\
& +\left\langle\overline{\tilde{\chi}}^{+}\left(A_{+}^{(0)}+A_{+}^{(1)}\right) \tilde{\chi}^{+}\right\rangle \\
& +\left\langle\overline{\tilde{\chi}}^{0} A_{0+}^{(1)} \tilde{\chi}^{+}\right\rangle+\left\langle\overline{\tilde{\chi}}^{+} A_{+0}^{(1)} \tilde{\chi}^{o}\right\rangle
\end{aligned}
$$

where,

$$
\begin{aligned}
\left\langle\overline{\tilde{\chi}}^{0} A_{0}^{(i)} \tilde{\chi}^{o}\right\rangle & \equiv \int d x d y \overline{\tilde{\chi}}_{x}^{0} A_{0 x y}^{(i)} \tilde{\chi}_{y}^{o}, \\
\left\langle\overline{\tilde{\chi}}^{+} A_{+}^{(i)} \tilde{\chi}^{+}\right\rangle & \equiv \int d x d y \overline{\tilde{\chi}}_{x}^{+} A_{+x y}^{(i)} \tilde{\chi}_{y}^{+} ; i=0,1, \\
\left\langle\overline{\tilde{\chi}}^{0} A_{0+}^{(1)} \tilde{\chi}^{+}\right\rangle & \equiv \int d x d y \overline{\tilde{\chi}}_{x}^{0} A_{0+x y}^{(1)} \tilde{\chi}_{y}^{+}, \\
\left\langle\overline{\tilde{\chi}}^{+} A_{+0}^{(1)} \tilde{\chi}^{o}\right\rangle & \equiv \int d x d y \overline{\tilde{\chi}}_{x}^{+} A_{+0 x y}^{(1)} \tilde{\chi}_{y}^{o},
\end{aligned}
$$

and the operators are:

$$
\begin{aligned}
A_{0 x y}^{(0)} & \equiv\left(i \not \partial-\tilde{M}^{0}\right)_{x} \delta_{x y}, A_{+x y}^{(0)} \equiv\left(i \not \partial-\tilde{M}^{+}\right)_{x} \delta_{x y} \\
A_{0 x y}^{(1)} & \equiv\left[\frac{g}{c_{w}} Z_{\mu} \gamma^{\mu}\left(O_{L}^{\prime \prime} P_{L}+O_{R}^{\prime \prime} P_{R}\right)\right]_{x} \delta_{x y} \\
A_{+x y}^{(1)} & \equiv\left[\frac{g}{c_{w}} Z_{\mu} \gamma^{\mu}\left(O_{L}^{\prime} P_{L}+O_{R}^{\prime} P_{R}\right)-e A_{\mu} \gamma^{\mu}\right]_{x} \delta_{x y} \\
A_{0+x y}^{(1)} & \equiv\left[g W_{\mu}^{-} \gamma^{\mu}\left(O_{L} P_{L}+O_{R} P_{R}\right)\right]_{x} \delta_{x y}, \\
A_{+0 x y}^{(1)} & \equiv\left[g W_{\mu}^{+} \gamma^{\mu}\left(O_{L}^{+} P_{L}+O_{R}^{+} P_{R}\right)\right]_{x} \delta_{x y}
\end{aligned}
$$

being $P_{L}=1 / 2\left(1-\gamma_{5}\right)$ and $P_{R}=1 / 2\left(1+\gamma_{5}\right)$.

Given all the ingredients, we now proceed with the integration. The formula of the effective action (60) can be factorized into three pieces:

$$
e^{i \Gamma_{e f f}[V]}=e^{i \Gamma_{o}[V]} e^{i \Gamma_{e f f}^{\tilde{f}}[V]} e^{i \Gamma_{e f f}^{\tilde{\chi}}[V]}
$$

where,

$$
\begin{gathered}
e^{i \Gamma_{e f f}^{\tilde{f}}[V]}=\int[d \tilde{f}]\left[d \tilde{f}^{*}\right] e^{i \Gamma_{\tilde{f}}[V, \tilde{f}]} \\
e^{i \Gamma_{e f f}^{\tilde{\chi}}[V]}=\int\left[d \tilde{\chi}^{+}\right]\left[d \overline{\tilde{\chi}}^{+}\right]\left[d \tilde{\chi}^{o}\right] e^{i \Gamma_{\tilde{\chi}}[V, \tilde{\chi}]}
\end{gathered}
$$

We next compute $\Gamma_{e f f}^{\tilde{f}}[V]$ and $\Gamma_{e f f}^{\tilde{\chi}}[V]$ separately. By substituting (62) into (70), and by performing a standard Gaussian integration we get,

$$
\begin{aligned}
\Gamma_{\text {eff }}^{\tilde{f}}[V] & =i \operatorname{Tr} \log A_{\tilde{f}} \\
& =i \operatorname{Tr} \log \left[A_{\tilde{f}}^{(o)}\left(1+A_{\tilde{f}}^{(o)-1}\left(A_{\tilde{f}}^{(1)}+A_{\tilde{f}}^{(2)}\right)\right)\right]
\end{aligned}
$$


The logarithm can be expanded and the $V$ independent terms can be left apart since they are irrevelant for the present computation. We get,

$$
\Gamma_{e f f}^{\tilde{f}}[V]=i \sum_{k=1}^{\infty} \frac{(-1)^{k+1}}{k} \operatorname{Tr}\left[G_{\tilde{f}}\left(A_{\tilde{f}}^{(1)}+A_{\tilde{f}}^{(2)}\right)\right]^{k}
$$

where, $G_{\tilde{f}} \equiv A_{\tilde{f}}^{(o)^{-1}}$ and represents the free sfermion propagator matrix in the position space,

$$
G_{\tilde{f} x y}^{i j} \equiv \int \frac{d^{D} q}{(2 \pi)^{D}} \mu_{o}^{4-D} e^{-i q(x-y)}\left(q^{2}-\tilde{M}_{f}^{2}\right)_{i j}^{-1}
$$

with,

$$
\begin{aligned}
& \left(q^{2}-\tilde{M}_{f}^{2}\right)^{-1} \\
& \quad=\operatorname{diag}\left(\frac{1}{q^{2}-\tilde{m}_{t_{1}}^{2}}, \frac{1}{q^{2}-\tilde{m}_{t_{2}}^{2}}, \frac{1}{q^{2}-\tilde{m}_{b_{1}}^{2}}, \frac{1}{q^{2}-\tilde{m}_{b_{2}}^{2}}\right)
\end{aligned}
$$

if $\quad \tilde{f}=\tilde{q} ;$ or

$$
\left(q^{2}-\tilde{M}_{f}^{2}\right)^{-1}=\operatorname{diag}\left(\frac{1}{q^{2}-\tilde{m}_{\nu}^{2}}, \frac{1}{q^{2}}, \frac{1}{q^{2}-\tilde{m}_{\tau_{1}}^{2}}, \frac{1}{q^{2}-\tilde{m}_{\tau_{2}}^{2}}\right)
$$

if $\quad \tilde{f}=\tilde{l}$,

and, as always, a sum over the three generations and over the $N_{c}$ colors of the squarks are understood.

Finally, if we keep just the terms that contribute to the two-point functions, the effective action generated from sfermions integration can be written as,

$$
\Gamma_{e f f}^{\tilde{f}}[V]=i \operatorname{Tr}\left(G_{\tilde{f}} A_{\tilde{f}}^{(2)}\right)-\frac{i}{2} \operatorname{Tr}\left(G_{\tilde{f}} A_{\tilde{f}}^{(1)}\right)^{2}+O\left(V^{3}\right)
$$

Notice that in the case of sleptons we have formally integrated over the four components of $\tilde{f}=\tilde{l}$. This integration, in principle, would include a rigth-handed sneutrino. However, due to the fact that this sneutrino doesn't couple to any of the gauge bosons (see the definitions of $\hat{Q}_{f}, \hat{G}_{f}, \Sigma_{f}, \Sigma_{f}^{t b}$ in $\left.(34)\right)$, it does not finally contribute to the effective action, as it must be.

We next compute the effective action generated from neutralinos and charginos integration. By substituting (66) into (71) we get,

$$
\begin{aligned}
& e^{i \Gamma_{e f f}^{\tilde{\chi}}[V]} \\
& =\int\left[d \tilde{\chi}^{+}\right]\left[d \overline{\tilde{\chi}}^{+}\right]\left[d \tilde{\chi}^{o}\right] \exp \left(i \left\{\frac{1}{2}\left\langle\overline{\tilde{\chi}}^{o}\left(A_{o}^{(o)}+A_{o}^{(1)}\right) \tilde{\chi}^{o}\right\rangle\right.\right. \\
& \left.\left.+\left\langle\overline{\tilde{\chi}}^{+}\left(A_{+}^{(o)}+A_{+}^{(1)}\right) \tilde{\chi}^{+}\right\rangle+\left\langle\overline{\tilde{\chi}}^{o} A_{o+}^{(1)} \tilde{\chi}^{+}\right\rangle+\left\langle\overline{\tilde{\chi}}^{+} A_{o+}^{(1)} \tilde{\chi}^{o}\right\rangle\right\}\right) .
\end{aligned}
$$

By performing first the integration over the charginos we find,

$$
\begin{aligned}
e^{i \Gamma_{e f f}^{\tilde{\tilde{\chi}}}[V]}= & \operatorname{det}\left(A_{+}^{(o)}+A_{+}^{(1)}\right) \int\left[d \tilde{\chi}^{o}\right] \\
& \times e^{i \frac{1}{2}\left\langle\overline{\tilde{\chi}}^{o}\left[A_{o}^{(o)}+A_{o}^{(1)}-2 A_{o+}^{(1)}\left(A_{+}^{(o)}+A_{+}^{(1)}\right)^{-1} A_{+o}^{(1)}\right] \tilde{\chi}^{o}\right\rangle}
\end{aligned}
$$

and next, by integrating over neutralinos we get,

$$
\begin{aligned}
& e^{i \Gamma_{e f f}^{\tilde{\chi}}[V]}=\operatorname{det}\left(A_{+}^{(o)}+A_{+}^{(1)}\right) \\
& \quad \times \operatorname{det}\left[A_{o}^{(o)}+A_{o}^{(1)}-2 A_{o+}^{(1)}\left(A_{+}^{(o)}+A_{+}^{(1)}\right)^{-1} A_{+o}^{(1)}\right]^{\frac{1}{2}} .
\end{aligned}
$$

The effective action can therefore be written as,

$$
\begin{aligned}
& \Gamma_{e f f}^{\tilde{\chi}}[V]=-i \operatorname{Tr} \log \left(A_{+}^{(o)}+A_{+}^{(1)}\right) \\
& \quad-\frac{i}{2} \operatorname{Tr} \log \left[A_{o}^{(o)}+A_{o}^{(1)}-2 A_{o+}^{(1)}\left(A_{+}^{(o)}+A_{+}^{(1)}\right)^{-1} A_{+o}^{(1)}\right] .
\end{aligned}
$$

These logarithms can be worked out as before. By ignoring the $V$-independent terms and by expanding the logarithm we find,

$$
\begin{aligned}
&-i \operatorname{Tr} \log \left(A_{+}^{(o)}+A_{+}^{(1)}\right) \\
&=-i \operatorname{Tr} \log \left[A_{+}^{(o)}\left(1+A_{+}^{(o)^{-1}} A_{+}^{(1)}\right)\right] \\
&=-i \operatorname{Tr} \log \left(1+k_{+} A_{+}^{(1)}\right) \\
&=-i \sum_{k=1}^{\infty} \frac{(-1)^{k+1}}{k} \operatorname{Tr}\left(k_{+} A_{+}^{(1)}\right)^{k},
\end{aligned}
$$

where $k_{+} \equiv A_{+}^{(o)^{-1}}$ and represents the free chargino propagator matrix in the position space,

$$
k_{+x y}^{i j} \equiv \int \frac{d^{D} q}{(2 \pi)^{D}} \mu_{o}^{4-D} e^{-i q(x-y)}\left(\not q-\tilde{M}_{+}\right)_{i j}^{-1},{ }_{(i, j=1,2)}
$$

Similarly, we find,

$$
\begin{aligned}
- & \frac{i}{2} \operatorname{Tr} \log \left[A_{o}^{(o)}+A_{o}^{(1)}-2 A_{o+}^{(1)}\left(A_{+}^{(o)}+A_{+}^{(1)}\right)^{-1} A_{+o}^{(1)}\right] \\
= & -\frac{i}{2} \operatorname{Tr} \log \left[A _ { o } ^ { ( o ) } \left(1+A_{o}^{(o)^{-1}} A_{o}^{(1)}\right.\right. \\
& \left.\left.-2 A_{o}^{(o)^{-1}} A_{o+}^{(1)}\left(A_{+}^{(o)}+A_{+}^{(1)}\right)^{-1} A_{+o}^{(1)}\right)\right] \\
= & -\frac{i}{2} \operatorname{Tr} \log \left[1+k_{o} A_{o}^{(1)}\right. \\
& \left.-2 k_{o} A_{o+}^{(1)}\left(1+k_{+} A_{+}^{(1)}\right)^{-1} k_{+} A_{+o}^{(1)}\right] \\
= & \left.-\frac{i}{2} \sum_{k=1}^{\infty} \frac{(-1)^{k+1}}{k} \operatorname{Tr}_{k} k_{o}^{(1)} A_{o}^{(1)}\right]^{k} \\
& \left.-2 k_{o} A_{o+}^{(1)} \sum_{r=0}^{\infty}(-1)^{r}\left(k_{+} A_{+}^{(1)}\right)^{r} k_{+}^{(1)}\right]^{k}
\end{aligned}
$$

where $k_{o} \equiv A_{o}^{(o)^{-1}}$ and represents the free neutralino propagator matrix in the position space,

$$
k_{o x y}^{i j} \equiv \int \frac{d^{D} q}{(2 \pi)^{D}} \mu_{o}^{4-D} e^{-i q(x-y)}\left(\not q-\tilde{M}_{o}\right)_{i j}^{-1},
$$


The sum of (77) and (79) gives the total contribution to the one-loop effective action generated from neutralino and chargino integration $\Gamma_{e f f}^{\tilde{\chi}}[V]$.

Finally, if we keep just the terms that contribute to the two points functions we obtain,

$$
\begin{aligned}
\Gamma_{e f f}^{\tilde{\chi}}[V]= & \frac{i}{2} \operatorname{Tr}\left(k_{+} A_{+}^{(1)}\right)^{2}+\frac{i}{4} \operatorname{Tr}\left(k_{o} A_{o}^{(1)}\right)^{2} \\
& +i \operatorname{Tr}\left(k_{o} A_{o+}^{(1)} k_{+} A_{+o}^{(1)}\right)+O\left(V^{3}\right) .
\end{aligned}
$$

The total resulting effective action for the two-point Green functions can be finally summarized by the following expression,

$$
\begin{aligned}
\Gamma_{e f f}[V]= & \Gamma_{o}[V]+\Gamma_{e f f}^{\tilde{f}}[V]+\Gamma_{e f f}^{\tilde{\chi}}[V] \\
= & \Gamma_{o}[V]+i \operatorname{Tr}\left(G_{\tilde{f}} A_{\tilde{f}}^{(2)}\right)-\frac{i}{2} \operatorname{Tr}\left(G_{\tilde{f}} A_{\tilde{f}}^{(1)}\right)^{2} \\
& +\frac{i}{2} \operatorname{Tr}\left(k_{+} A_{+}^{(1)}\right)^{2}+\frac{i}{4} \operatorname{Tr}\left(k_{o} A_{o}^{(1)}\right)^{2} \\
& +i \operatorname{Tr}\left(k_{o} A_{o+}^{(1)} k_{+} A_{+o}^{(1)}\right)+O\left(V^{3}\right)
\end{aligned}
$$

Diagrammatically, $\Gamma_{o}$ gives the contribution to the free two-point functions, the second and third terms give the two types of one loop contributions with all kind of sfermions in the loop, as in Fig. 1a, the fourth term gives the one-loop contributions with charginos in the loop, the fifth term is the corresponding contribution with neutralinos in the loop and the last term gives the mixed one-loop contributions with both charginos and neutralinos in the loop. The corresponding diagrams to the last three terms are shown in Fig. 1b.

\section{Two-point functions for the electroweak gauge bosons in the large SUSY masses limit}

In this section we present the two point functions for the electroweak gauge bosons to one loop and analize the limit of large masses of the SUSY particles.

In order to get the explicit expressions for the twopoint functions one must work out the traces in the formula (82). This involves lengthy algebraic standard manipulations that we do not present here for brevity. Basically one must substitute into (82) the operators $A$ and propagators $G$ and $K$ of $(64,68,73,78,80)$, express the one-loop integrals in momentum space of $\mathrm{D}$ dimensions, compute all the appearing Dirac traces and Fourier transform the result back to the position space. The traces also involve to perform the sum in the corresponding matrix indexes, the sum over the various types of sfermions and the sum in color indexes in the case of squarks. The result for the effective action is the following:

$$
\begin{aligned}
\Gamma_{e f f}[V]= & \frac{1}{2} \int d x d y A_{x}^{\mu} \Gamma_{\mu \nu}^{A A}(x, y) A_{y}^{\nu} \\
& +\frac{1}{2} \int d x d y Z_{x}^{\mu} \Gamma_{\mu \nu}^{Z Z}(x, y) Z_{y}^{\nu}
\end{aligned}
$$

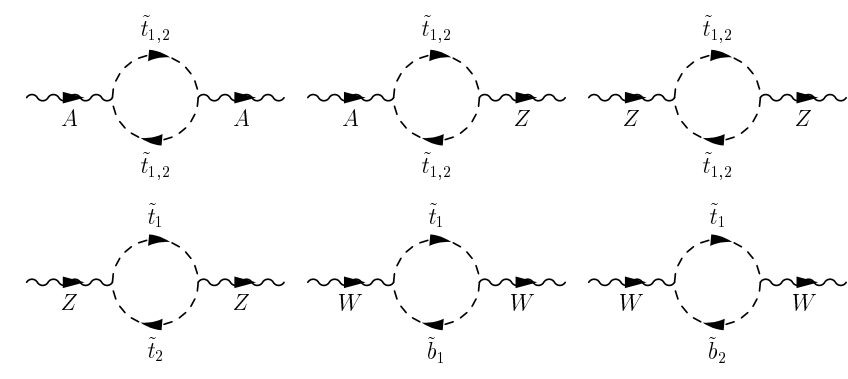

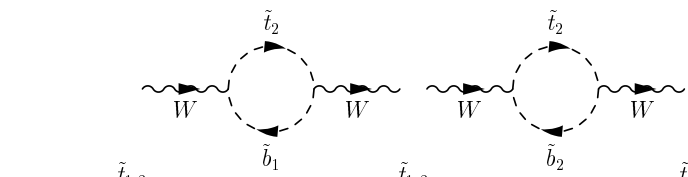

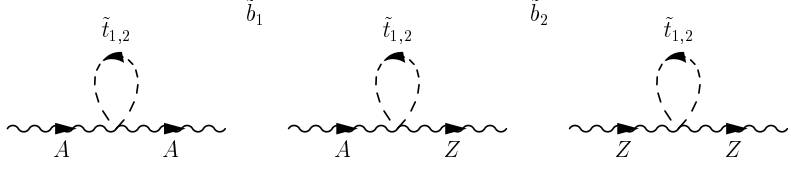

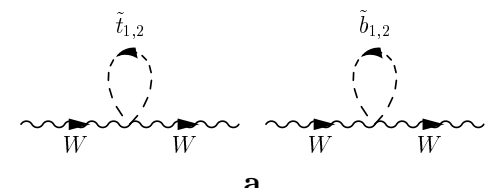

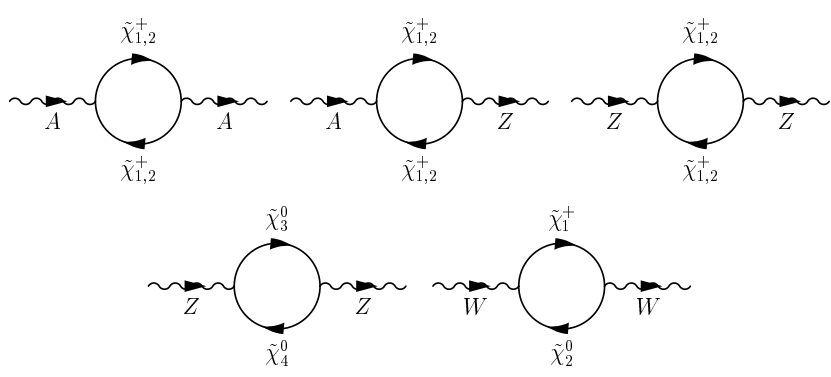

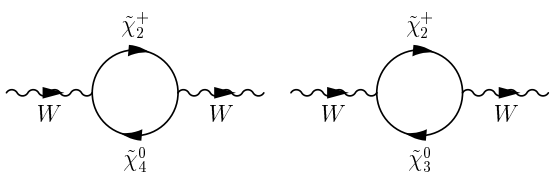

b

Fig. 1. a Feynman diagrams with scalars in the loops that contribute to the two-point functions of the electroweak gauge bosons. The diagrams shown for neutral gauge bosons are of stop loops. Not shown are the similar diagrams of sbottom loops. For the sleptons sector, $\nu, \tilde{\tau}_{1}$ and $\tilde{\tau}_{2}$, analogous diagrams are obtained. b Feynman diagrams with charginos and neutralinos in the loops that dominate the two-point functions of electroweak gauge bosons in the asymptotic limit of very large ino masses

$$
\begin{aligned}
& +\int d x d y A_{x}^{\mu} \Gamma_{\mu \nu}^{A Z}(x, y) Z_{y}^{\nu} \\
& +\int d x d y W_{x}^{+\mu} \Gamma_{\mu \nu}^{+-}(x, y) W_{y}^{-\nu}+O\left(V^{3}\right)
\end{aligned}
$$

where $\Gamma_{\mu \nu}(x, y)$ are the two-point functions in position space. Their relation with the corresponding functions in 
momentum space is defined by,

$$
(2 \pi)^{4} \delta\left(k_{1}+k_{2}\right) \Gamma_{\mu \nu}\left(k_{1}\right) \equiv \int d x d y e^{-i k_{1} x-i k_{2} y} \Gamma_{\mu \nu}(x, y) .
$$

Finally, for the two-point functions in momentum space we find,

$$
\begin{aligned}
& \Gamma_{\mu \nu}^{A A}(k)=-k^{2} g_{\mu \nu}+\left(1-\frac{1}{\xi_{A}}\right) k_{\mu} k_{\nu} \\
& +i e^{2} \sum_{\tilde{f}}\left\{2 \sum_{a} I_{o}\left(\tilde{m}_{f_{a}}^{2}\right)\left(\hat{Q}_{f}^{2}\right)_{a a} g_{\mu \nu}\right. \\
& \left.-\sum_{a b}\left(\hat{Q}_{f}\right)_{a b}\left(\hat{Q}_{f}\right)_{b a} I_{f_{\mu \nu}}^{a b}\left(k, \tilde{m}_{f_{a}}, \tilde{m}_{f_{b}}\right)\right\} \\
& +2 i e^{2} \sum_{i=1}^{2}\left\{T_{\mu \nu}^{i i}\left(k, \tilde{M}_{i}^{+}, \tilde{M}_{i}^{+}\right)+2 \hat{I}^{i i}\left(k, \tilde{M}_{i}^{+}, \tilde{M}_{i}^{+}\right) g_{\mu \nu}\right\} \\
& \Gamma_{\mu \nu}^{Z Z}(k)=\left(m_{Z}^{2}-k^{2}\right) g_{\mu \nu}+\left(1-\frac{1}{\xi_{Z}}\right) k_{\mu} k_{\nu} \\
& +i \frac{g^{2}}{c_{W}^{2}} \sum_{\tilde{f}}\left\{2 \sum_{a} I_{o}\left(\tilde{m}_{f_{a}}^{2}\right)\left(\hat{G}_{f}^{2}\right)_{a a} g_{\mu \nu}\right. \\
& \left.-\sum_{a b}\left(\hat{G}_{f}\right)_{a b}\left(\hat{G}_{f}\right)_{b a} I_{f_{\mu \nu}}^{a b}\left(k, \tilde{m}_{f_{a}}, \tilde{m}_{f_{b}}\right)\right\} \\
& +\frac{i}{2} \frac{g^{2}}{c_{W}^{2}} \sum_{i, j=1}^{4}\left\{\left(O_{L}^{\prime \prime}{ }_{L}^{i j} O_{L}^{\prime \prime j i}+O_{R}^{\prime \prime i j} O_{R}^{\prime \prime j i}\right) T_{\mu \nu}^{i j}\left(k, \tilde{M}_{i}^{0}, \tilde{M}_{j}^{0}\right)\right. \\
& \left.+2\left(O_{L}^{\prime \prime i j} O_{R}^{\prime \prime j i}+O_{R}^{\prime \prime i j} O_{L}^{\prime \prime j i}\right) \hat{I}^{i j}\left(k, \tilde{M}_{i}^{0}, \tilde{M}_{j}^{0}\right) g_{\mu \nu}\right\} \\
& +i \frac{g^{2}}{c_{W}^{2}} \sum_{i, j=1}^{2}\left\{\left(O_{L}^{\prime i j} O_{L}^{\prime j i}+O_{R}^{\prime i j} O_{R}^{\prime j i}\right) T_{\mu \nu}^{i j}\left(k, \tilde{M}_{i}^{+}, \tilde{M}_{j}^{+}\right)\right. \\
& \left.+2\left(O_{L}^{\prime i j} O_{R}^{\prime j i}+O_{R}^{\prime i j} O_{L}^{\prime j i}\right) \hat{I}^{i j}\left(k, \tilde{M}_{i}^{+}, \tilde{M}_{j}^{+}\right) g_{\mu \nu}\right\} \\
& \Gamma_{\mu \nu}^{A Z}(k)=\Gamma_{\mu \nu}^{Z A}(k) \\
& =\frac{i g e}{c_{W}} \sum_{\widetilde{f}}\left\{2 \sum_{a} I_{0}\left(\tilde{m}_{f_{a}}^{2}\right)\left(\widehat{Q}_{f} \widehat{G}_{f}\right)_{a a} g_{\mu \nu}\right. \\
& \left.-\sum_{a b}\left(\widehat{Q}_{f}\right)_{a b}\left(\widehat{G}_{f}\right)_{b a} I_{f_{\mu \nu} b}^{a b}\left(k, \tilde{m}_{f_{a}}, \tilde{m}_{f_{b}}\right)\right\} \\
& -\frac{i g e}{c_{W}} \sum_{i=1}^{2}\left(\begin{array}{c}
{O^{\prime}}_{L}^{i i} \\
+O_{R}^{\prime i i}
\end{array}\right)\left(T_{\mu \nu}^{i i}\left(k, \tilde{M}_{i}^{+}, \tilde{M}_{i}^{+}\right)\right. \\
& \left.+2 \hat{I}^{i i}\left(k, \tilde{M}_{i}^{+}, \tilde{M}_{i}^{+}\right) g_{\mu \nu}\right)
\end{aligned}
$$

$\Gamma_{\mu \nu}^{+-}(k)=\Gamma_{\mu \nu}^{-+}(k)=\left(m_{W}^{2}-k^{2}\right) g_{\mu \nu}+\left(1-\frac{1}{\xi_{W}}\right) k_{\mu} k_{\nu}$ $+\frac{i g^{2}}{2} \sum_{\widetilde{f}}\left\{\sum_{a}\left(\Sigma_{f}\right)_{a a} I_{0}\left(\tilde{m}_{f_{a}}^{2}\right) g_{\mu \nu}\right.$

$$
\begin{aligned}
& \left.-\sum_{a, b}\left(\Sigma_{f}^{t b}\right)_{a b}\left(\Sigma_{f}^{t b}\right)_{a b} I_{f_{\mu \nu}}^{a b}\left(k, \tilde{m}_{f_{a}}, \tilde{m}_{f_{b}}\right)\right\} \\
& +i g^{2} \sum_{i=1}^{4} \sum_{j=1}^{2}\left\{\left(O_{L}^{i j} O_{L}^{+j i}+O_{R}^{i j} O_{R}^{+j i}\right) T_{\mu \nu}^{i j}\left(k, \tilde{M}_{i}^{0}, \tilde{M}_{j}^{+}\right)\right. \\
& \left.+2\left(O_{L}^{i j} O_{R}^{+j i}+O_{R}^{i j} O_{L}^{+j i}\right) \hat{I}^{i j}\left(k, \tilde{M}_{i}^{0}, \tilde{M}_{j}^{+}\right) g_{\mu \nu}\right\}
\end{aligned}
$$

Here the indexes $a$ and $b$ run over the four entries of the sfermions column matrix in (30), and the sum in $\tilde{f}$ refers to the sum over squarks and sleptons of each generation as well as to the sum in color indexes for the squarks case. The indexes $i, j$ vary as $i, j=1,2,3,4$ if they refer to neutralinos and as $i, j=1,2$ if they refer to charginos.

The one-loop integrals in (84) through (87) are defined in dimensional regularization by,

$$
I_{0}\left(\tilde{m}_{f a}^{2}\right)=\int d \widehat{q} \frac{1}{\left[q^{2}-\tilde{m}_{f a}^{2}\right]}
$$

$$
I_{f_{\mu \nu}}^{a b}\left(k, \tilde{m}_{f_{a}}, \tilde{m}_{f_{b}}\right)=\int d \widehat{q} \frac{(2 q+k)_{\mu}(2 q+k)_{\nu}}{\left[(k+q)^{2}-\tilde{m}_{f_{a}}^{2}\right]\left[q^{2}-\tilde{m}_{f_{b}}^{2}\right]}
$$

$$
I^{i j}\left(k, \tilde{M}_{i}, \tilde{M}_{j}\right)=\int d \widehat{q} \frac{1}{\left[q^{2}-\tilde{M}_{i}^{2}\right]\left[(q+k)^{2}-\tilde{M}_{j}^{2}\right]}
$$

$$
\begin{aligned}
& T_{\mu \nu}^{i j}=T_{\nu \mu}^{j i}=4 I_{\mu \nu}^{i j}-2 g_{\mu \nu} g^{\alpha \beta} I_{\alpha \beta}^{i j}+2\left({I^{\prime}}_{\mu \nu}^{i j}+{I^{\prime}}_{\nu \mu}^{i j}\right) \\
& -2 g_{\mu \nu} g^{\alpha \beta}{I^{\prime \prime \beta}}^{i j} \\
& I_{\mu \nu}^{i j}\left(k, \tilde{M}_{i}, \tilde{M}_{j}\right)=\int d \widehat{q} \frac{q_{\mu} q_{\nu}}{\left[q^{2}-\tilde{M}_{i}^{2}\right]\left[(k+q)^{2}-\tilde{M}_{j}^{2}\right]}
\end{aligned}
$$$$
{I^{\prime}}_{\mu \nu}^{i j}\left(k, \tilde{M}_{i}, \tilde{M}_{j}\right)=\int d \widehat{q} \frac{q_{\mu} k_{\nu}}{\left[q^{2}-\tilde{M}_{i}^{2}\right]\left[(k+q)^{2}-\tilde{M}_{j}^{2}\right]}
$$

$$
\hat{I}^{i j}\left(k, \tilde{M}_{i}, \tilde{M}_{j}\right)=\int d \widehat{q} \frac{\tilde{M}_{i} \tilde{M}_{j}}{\left[q^{2}-\tilde{M}_{i}^{2}\right]\left[(k+q)^{2}-\tilde{M}_{j}^{2}\right]}
$$

Here one extra integral $I^{i j}$ has been included for completeness. In the above integrals $\tilde{M}_{i}$ should be understood as $\tilde{M}_{i}^{0}$ if the index $\mathrm{i}(\mathrm{i}=1, \ldots, 4)$ refers to neutralinos or as $\tilde{M}_{i}^{+}$if the index $\mathrm{i}(\mathrm{i}=1,2)$ refers to charginos, and:

$$
\int d \widehat{q} \equiv \int \frac{d^{D} q}{(2 \pi)^{D}} \mu_{o}^{4-D} .
$$

Notice, that for the kind of loop integrals that we are considering there should not be relevant difference in the results with respect to other regularization methods, as for instance, dimensional reduction. We have not done, however, this check explicitly. 
The self-energies $\Sigma^{X Y}$ are defined from the two-point functions as usual,

$$
\begin{aligned}
\Gamma_{\mu \nu}^{X Y}(k)= & \Gamma_{0}^{X Y}(k) \\
& +\Sigma^{X Y}(k) g_{\mu \nu}+R^{X Y}(k) k_{\mu} k_{\nu}
\end{aligned}
$$

where $\Gamma_{0 \mu \nu}^{X Y}$ are the two-point functions at tree level and $X, Y=A, Z, W^{ \pm}$.

The transverse and longitudinal parts of the two-point functions, $\Sigma_{T}^{X Y}$ and $\Sigma_{L}^{X Y}$, are defined by,

$$
\begin{aligned}
\Gamma_{\mu \nu}^{X Y}(k)= & \Gamma_{0 \mu \nu}^{X Y}(k) \\
& +\Sigma_{T}^{X Y}(k)\left(g_{\mu \nu}-\frac{k_{\mu} k_{\nu}}{k^{2}}\right)+\Sigma_{L}^{X Y}(k) \frac{k_{\mu} k_{\nu}}{k^{2}}
\end{aligned}
$$

The expressions for the self energies $\Sigma^{X Y}(k)$ and the $R^{X Y}(k)$ functions can be easily read from (84) through (87). It is convenient to express them in terms of new functions $A^{\prime} s, B^{\prime} s, C^{\prime} s$ and $D^{\prime} s$ being defined as follows,

$$
\begin{aligned}
I_{f_{\mu \nu}}^{a b}\left(k, \tilde{m}_{f_{a}}, \tilde{m}_{f_{b}}\right)= & A_{f}^{a b}\left(k, \tilde{m}_{f_{a}}, \tilde{m}_{f_{b}}\right) g_{\mu \nu} \\
& +B_{f}^{a b}\left(k, \tilde{m}_{f_{a}}, \tilde{m}_{f_{b}}\right) k_{\mu} k_{\nu} \\
T_{\mu \nu}^{i j}\left(k, \tilde{M}_{i}, \tilde{M}_{j}\right)= & C^{i j}\left(k, \tilde{M}_{i}, \tilde{M}_{j}\right) g_{\mu \nu} \\
& +D^{i j}\left(k, \tilde{M}_{i}, \tilde{M}_{j}\right) k_{\mu} k_{\nu} .
\end{aligned}
$$

The results for $\Sigma^{X Y}(k)$ and $R^{X Y}(k)$ are collected in Appendix A. This complete our computation of the effective action and the two-point functions for gauge bosons which should be noticed are exact to one-loop.

Since we are interested in the large mass limit of the SUSY particles we need to have at hand not just the exact results of the above integrals but their asymptotic expressions to be valid in that limit. We will present in the following the asymptotic results of the two-point functions and the gauge bosons self-energies.

We have analized the integrals by means of the socalled m-Theorem [23]. This theorem provides a powerful technique to study the asymptotic behaviour of Feynman integrals in the limit where some of the masses are large. Notice that this is not trivial since some of these integrals are divergent and the interchange of the integral with the limit is not allowed. Thus, one should first compute the integrals in dimensional regularization and at the end take the large mass limit. Instead of this direct way it is also possible to proceed as follows: First, one rearranges the integrand through algebraic manipulations in order to decrease the ultraviolet divergence degree of some parts of the integral up to separate the MS (or DR) regularized integral into two parts, one of which is a divergent contribution in 4 dimensions that can be evaluated exactly using the standard techniques, and the other one is a convergent part which satisfies the requirements demanded by the mTheorem and therefore, goes to zero in the infinite mass limit. By means of this procedure we guarantee rigurously the correct asymptotic behaviour of the integrals.
We give the details of the computation of the Feynman integrals by means of the $\mathrm{m}$-Theorem in Appendix B. These integrals have been used to obtain the final results for the transverse and longitudinal parts, $\Sigma_{T}^{X Y}(k)$ and $\Sigma_{L}^{X Y}(k)$, given in Appendix C.

Some comments on these results are in order:

- These asymptotic expressions are completely general and depend just on the physical masses of the SUSY particles and on the generic coefficients $c_{q}, s_{q}, c_{l}, s_{l}$, $O_{L, R}^{j i}, O_{L, R}^{\prime j i}, O_{L, R}^{\prime \prime j i}$. Notice that they do not depend on the particular mechanism that generates the SUSY masses.

- We have done this computation, in addition, by diagrammatical methods and we have found the same results. It involves the evaluation of Feynman diagrams with all kind of sparticles in the loops. We have shown in Fig. 1 the various diagrams contributing to the twopoint functions $\Gamma^{A A}, \Gamma^{A Z}, \Gamma^{Z Z}$ and $\Gamma^{W W}$. This diagrammatic computation provides a good check of our previous results from functional methods, and at the same time helps to illustrate which sparticle masses must be compared to which in the large mass expansion. Notice that the longitudinal components of the two-point functions involving the photon field fulfil the expected Slavnov-Taylor identities.

- In all these asymptotic expressions, the physical sparticle masses are assumed to be much larger than the external momenta. As we can see, from Fig. 1, the masses that must be compared to each other are the ones appering in the same one-loop diagram. Thus, for instance, the self-energies $\Sigma^{A A}$ and $\Sigma^{A Z}$, where no mixed diagrams with different sfermions contribute, do not need of any reference on the relative size of the sfermion masses. $\Sigma^{Z Z}$ and $\Sigma^{W W}$, on the contrary, do require this comparison. In the case of $\Sigma^{Z Z}$ one needs to compare squarks of the same charge, sleptons of the same charge, charginos of the same charge and neutralinos among them. No comparison among sfermions of different generations is required since we have not considered intergenerational mixing in this paper. In the case of $\Sigma^{W W}$ one needs to compare, in each generation, the squarks of different charge, the sleptons of different charge and the netralinos with the charginos. The realistic and more interesting situation will be when all the sparticles masses must be compared at the same time and, obviously, the final result will depend on the kind of SUSY hierarchy masses that had been previously established. This will happen in the observables where all the four self-energies do contribute.

- As discussed in the introduction, decoupling of heavy SUSY particles in the Appelquist-Carazzone Theorem sense will occur if the virtual effects due to these particles on the effective low-energy SM action can be absorbed into a redefinition of the SM parameters and wave functions renormalization, or else they are suppressed by inverse powers of the heavy SUSY particle masses. From our results in Appendix $\mathrm{C}$ it is clear that we get indeed decoupling in the two point elec- 
troweak gauge boson functions. This can be easily understood due to the specific analytical form of our formulae given generically by

$$
\Sigma^{X Y}(k)=\Sigma_{(0)}^{X Y}+\Sigma_{(1)}^{X Y} k^{2}
$$$$
\text { and } \quad R^{X Y}(k)=R_{(0)}^{X Y} \text {, }
$$

where $\Sigma_{(0)}^{X Y}, \Sigma_{(1)}^{X Y}$ and $R_{(0)}^{X Y}$ are functions of the SUSY large masses but are $k$ independent.

Equivalently, the transverse and longitudinal twopoint functions of Appendix C fulfil

$$
\begin{array}{ll} 
& \Sigma_{T}^{X Y}=\Sigma_{T(0)}^{X Y}+\Sigma_{T(1)}^{X Y} k^{2} \\
\text { and } & \left(\Sigma_{L}^{X Y}-\Sigma_{T}^{X Y}\right) \propto k^{2},
\end{array}
$$

which together are sufficient conditions to get decoupling.

\section{Decoupling of sparticles in $S, T$ and $U$}

The radiative corrections from SUSY particles to the observables $S, T$ and $U$ have been analyzed exhaustively in the literature $[6,7]$, but neither their complete analytical expressions in the large sparticle masses limit nor a general and systematic study of sparticles decoupling have been provided so far. We present in this section our results to one loop for these analytical expressions of $S, T$ and $U$ in a complete general form. Next we analyze under which particular conditions the sparticles decoupling takes place and finally we discuss how and why does it occur in the very special case of the MSSM with soft SUSY breaking terms.

The definition that we use for $S, T$ and $U$ are the usual ones [25]:

$$
\begin{aligned}
& S=-\frac{16 \pi}{e^{2}} s_{W} c_{W}\left[s_{W} c_{W} \Sigma_{A A}^{\prime}(0)-s_{W} s_{W} \Sigma_{Z Z}^{\prime}(0)\right. \\
&\left.+\left(c_{W}^{2}-s_{W}^{2}\right) \Sigma_{A Z}^{\prime}(0)\right] \\
& T=\frac{4 \pi}{e^{2}}\left[\frac{\Sigma_{W W}(0)}{m_{W}^{2}}-\frac{\Sigma_{Z Z}(0)}{m_{Z}^{2}}-2 \frac{s_{W}}{c_{W}} \frac{\Sigma_{A Z}(0)}{m_{Z}^{2}}\right] \\
& U= \frac{16 \pi}{e^{2}}\left[\Sigma_{W W}^{\prime}(0)-c_{W}{ }^{2} \Sigma_{Z Z}^{\prime}(0)-s_{W}{ }^{2} \Sigma_{A A}^{\prime}(0)\right. \\
&\left.\quad-2 s_{W} c_{W} \Sigma_{A Z}^{\prime}(0)\right] .
\end{aligned}
$$

The contribution to $S, T$ and $U$ are known to be finite and well defined separately for each sparticle sector, so that we can analyze them separately as well. As we have already said we consider in this paper all the sparticle contributions except that of the Higgs sector. Consequently, we define:

$$
\begin{aligned}
& S_{\mathrm{SUSY}}=S_{\tilde{q}}+S_{\tilde{l}}+S_{\tilde{\chi}}+S_{H} \\
& T_{\mathrm{SUSY}}=T_{\tilde{q}}+T_{\tilde{l}}+T_{\tilde{\chi}}+T_{H} \\
& U_{\mathrm{SUSY}}=U_{\tilde{q}}+U_{\tilde{l}}+U_{\tilde{\chi}}+U_{H}
\end{aligned}
$$

By using the corresponding expressions for the self-energies given in Appendix $\mathrm{C}$ we obtain the following results in the large masses limit, $m_{\tilde{q}_{i}}^{2}, m_{\tilde{l}_{j}}^{2}, \tilde{M}_{l}^{+^{2}}, \tilde{M}_{m}^{o^{2}} \gg k^{2}$, $\forall i, j, l, m$ and $k$ being the external momentum.

\subsection{Squarks}

Under the conditions:

$$
\begin{gathered}
\left|\tilde{m}_{t_{1}}^{2}-\tilde{m}_{t_{2}}^{2}\right| \ll\left|\tilde{m}_{t_{1}}^{2}+\tilde{m}_{t_{2}}^{2}\right|, \\
\left|\tilde{m}_{b_{1}}^{2}-\tilde{m}_{b_{2}}^{2}\right| \ll\left|\tilde{m}_{b_{1}}^{2}+\tilde{m}_{b_{2}}^{2}\right|
\end{gathered}
$$

we get,

$$
\begin{aligned}
S_{\tilde{q}}= & -\sum_{\tilde{q}} \frac{N_{c}}{36 \pi}\left\{\log \frac{\tilde{m}_{t_{1}}^{2}}{\tilde{m}_{b_{1}}^{2}}+s_{b}^{2} \log \frac{\tilde{m}_{b_{1}}^{2}}{\tilde{m}_{b_{2}}^{2}}-s_{t}^{2} \log \frac{\tilde{m}_{t_{1}}^{2}}{\tilde{m}_{t_{2}}^{2}}\right. \\
& -3 c_{t}^{2} s_{t}^{2} \log \frac{\left(\tilde{m}_{t_{1}}^{2}+\tilde{m}_{t_{2}}^{2}\right)^{2}}{4 \tilde{m}_{t_{1}}^{2} \tilde{m}_{t_{2}}^{2}} \\
& \left.-3 c_{b}^{2} s_{b}^{2} \log \frac{\left(\tilde{m}_{b_{1}}^{2}+\tilde{m}_{b_{2}}^{2}\right)^{2}}{4 \tilde{m}_{b_{1}}^{2} \tilde{m}_{b_{2}}^{2}}\right\} .
\end{aligned}
$$

For the observables $T_{\tilde{q}}$ and $U_{\tilde{q}}$ we must consider together:

$$
\begin{aligned}
\left|\tilde{m}_{t_{1}}^{2}-\tilde{m}_{t_{2}}^{2}\right| & \ll\left|\tilde{m}_{t_{1}}^{2}+\tilde{m}_{t_{2}}^{2}\right|, \\
\left|\tilde{m}_{b_{1}}^{2}-\tilde{m}_{b_{2}}^{2}\right| & \ll\left|\tilde{m}_{b_{1}}^{2}+\tilde{m}_{b_{2}}^{2}\right|, \\
\left|\tilde{m}_{t_{i}}^{2}-\tilde{m}_{b_{j}}^{2}\right| & \ll\left|\tilde{m}_{t_{i}}^{2}+\tilde{m}_{b_{j}}^{2}\right| \quad i, j=1,2
\end{aligned}
$$

obtaining,

$$
\begin{aligned}
T_{\tilde{q}}= & \sum_{\tilde{q}} \frac{N_{c}}{8 \pi} \frac{1}{s_{W}^{2} m_{W}^{2}}\left\{c_{t}^{2} c_{b}^{2} h\left(\tilde{m}_{t_{1}}^{2}, \tilde{m}_{b_{1}}^{2}\right)+c_{t}^{2} s_{b}^{2} h\left(\tilde{m}_{t_{1}}^{2}, \tilde{m}_{b_{2}}^{2}\right)\right. \\
& -c_{t}^{2} s_{t}^{2} h\left(\tilde{m}_{t_{1}}^{2}, \tilde{m}_{t_{2}}^{2}\right)+s_{t}^{2} c_{b}^{2} h\left(\tilde{m}_{t_{2}}^{2}, \tilde{m}_{b_{1}}^{2}\right) \\
& \left.-s_{t}^{2} s_{b}^{2} h\left(\tilde{m}_{t_{2}}^{2}, \tilde{m}_{b_{2}}^{2}\right)+s_{b}^{2} c_{b}^{2} h\left(\tilde{m}_{b_{1}}^{2}, \tilde{m}_{b_{2}}^{2}\right)\right\} \\
U_{\tilde{q}}= & \sum_{\tilde{q}} \frac{N_{c}}{12 \pi}\left\{c_{t}^{2} c_{b}^{2} \log \frac{4 \tilde{m}_{t_{1}}^{2} \tilde{m}_{b_{1}}^{2}}{\left(\tilde{m}_{t_{1}}^{2}+\tilde{m}_{b_{1}}^{2}\right)^{2}}\right. \\
& +c_{t}^{2} s_{b}^{2} \log \frac{4 \tilde{m}_{t_{1}}^{2} \tilde{m}_{b_{2}}^{2}}{\left(\tilde{m}_{t_{1}}^{2}+\tilde{m}_{b_{2}}^{2}\right)^{2}}+s_{t}^{2} s_{b}^{2} \log \frac{4 \tilde{m}_{t_{2}}^{2} \tilde{m}_{b_{2}}^{2}}{\left(\tilde{m}_{t_{2}}^{2}+\tilde{m}_{b_{2}}^{2}\right)^{2}} \\
& +s_{t}^{2} c_{b}^{2} \log \frac{4 \tilde{m}_{t_{2}}^{2} \tilde{m}_{b_{1}}^{2}}{\left(\tilde{m}_{t_{2}}^{2}+\tilde{m}_{b_{1}}^{2}\right)^{2}}-c_{t}^{2} s_{t}^{2} \log \frac{4 \tilde{m}_{t_{1}}^{2} \tilde{m}_{t_{2}}^{2}}{\left(\tilde{m}_{t_{1}}^{2}+\tilde{m}_{t_{2}}^{2}\right)^{2}} \\
& \left.-s_{b}^{2} c_{b}^{2} \log \frac{4 \tilde{m}_{b_{1}}^{2} \tilde{m}_{b_{2}}^{2}}{\left(\tilde{m}_{b_{1}}^{2}+\tilde{m}_{b_{2}}^{2}\right)^{2}}\right\},
\end{aligned}
$$

where:

$$
h\left(m_{1}^{2}, m_{2}^{2}\right) \equiv m_{1}^{2} \log \frac{2 m_{1}^{2}}{m_{1}^{2}+m_{2}^{2}}+m_{2}^{2} \log \frac{2 m_{2}^{2}}{m_{1}^{2}+m_{2}^{2}} .
$$

\subsection{Sleptons}

If $\left|\tilde{m}_{\tau_{1}}^{2}-\tilde{m}_{\tau_{2}}^{2}\right| \ll\left|\tilde{m}_{\tau_{1}}^{2}+\tilde{m}_{\tau_{2}}^{2}\right|:$

$$
S_{\tilde{l}}=-\sum_{\tilde{l}} \frac{1}{36 \pi}\left\{\log \frac{\tilde{m}_{\nu}^{2}}{\tilde{m}_{\tau_{1}}^{2}}+s_{\tau}^{2} \log \frac{\tilde{m}_{\tau_{1}}^{2}}{\tilde{m}_{\tau_{2}}^{2}}\right.
$$




$$
\begin{aligned}
& \left.-3 c_{\tau}^{2} s_{\tau}^{2} \log \frac{\left(\tilde{m}_{\tau_{1}}^{2}+\tilde{m}_{\tau_{2}}^{2}\right)^{2}}{4 \tilde{m}_{\tau_{1}}^{2} \tilde{m}_{\tau_{2}}^{2}}\right\} \\
T_{\tilde{l}}= & \sum_{\tilde{l}} \frac{1}{8 \pi} \frac{1}{s_{W}^{2} m_{W}^{2}}\left\{c_{\tau}^{2} h\left(\tilde{m}_{\nu}^{2}, \tilde{m}_{\tau_{1}}^{2}\right)+s_{\tau}^{2} h\left(\tilde{m}_{\nu}^{2}, \tilde{m}_{\tau_{2}}^{2}\right)\right. \\
& \left.+s_{\tau}^{2} c_{\tau}^{2} h\left(\tilde{m}_{\tau_{1}}^{2}, \tilde{m}_{\tau_{2}}^{2}\right)\right\} \\
U_{\tilde{l}}= & \sum_{\tilde{l}} \frac{1}{12 \pi}\left\{c_{\tau}^{2} \log \frac{4 \tilde{m}_{\nu}^{2} \tilde{m}_{\tau_{1}}^{2}}{\left(\tilde{m}_{\nu}^{2}+\tilde{m}_{\tau_{1}}^{2}\right)^{2}}\right. \\
& \left.+s_{\tau}^{2} \log \frac{4 \tilde{m}_{\nu}^{2} \tilde{m}_{\tau_{2}}^{2}}{\left(\tilde{m}_{\nu}^{2}+\tilde{m}_{\tau_{2}}^{2}\right)^{2}}-s_{\tau}^{2} c_{\tau}^{2} \log \frac{4 \tilde{m}_{\tau_{1}}^{2} \tilde{m}_{\tau_{2}}^{2}}{\left(\tilde{m}_{\tau_{1}}^{2}+\tilde{m}_{\tau_{2}}^{2}\right)^{2}}\right\}
\end{aligned}
$$

where the last two equations have been obtained considering in addition:

$$
\left|\tilde{m}_{\nu}^{2}-\tilde{m}_{\tau_{i}}^{2}\right| \ll\left|\tilde{m}_{\nu}^{2}+\tilde{m}_{\tau_{i}}^{2}\right| \quad i=1,2 .
$$

\subsection{Neutralinos and charginos}

$$
\begin{aligned}
& \text { If }\left|\tilde{M}_{1}^{+^{2}}-\tilde{M}_{2}^{+^{2}}\right| \ll\left|\tilde{M}_{1}^{+^{2}}+\tilde{M}_{2}^{+^{2}}\right| \text {, } \\
& \left|\tilde{M}_{i}^{o^{2}}-\tilde{M}_{j}^{o^{2}}\right| \ll\left|\tilde{M}_{i}^{o^{2}}+\tilde{M}_{j}^{o^{2}}\right| \quad i, j=1,2,3,4: \\
& S_{\tilde{\chi}}=-\frac{1}{3 \pi} \log \frac{2 \tilde{M}_{2}^{+^{2}}}{\tilde{M}_{3}^{o^{2}}+\tilde{M}_{4}^{o^{2}}}, \\
& T_{\tilde{\chi}}=\frac{1}{4 \pi m_{W}^{2} s_{W}{ }^{2}}\left\{-2\left(\tilde{M}_{1}^{+}-\tilde{M}_{2}^{o}\right)^{2} \log \frac{\tilde{M}_{1}^{+^{2}}+\tilde{M}_{2}^{o^{2}}}{2 \mu_{o}^{2}}\right. \\
& -\frac{1}{2}\left(\tilde{M}_{2}^{+}-\tilde{M}_{3}^{o}\right)^{2} \log \frac{\tilde{M}_{2}^{+^{2}}+\tilde{M}_{3}^{o^{2}}}{2 \mu_{o}^{2}} \\
& -\frac{1}{2}\left(\tilde{M}_{2}^{+}-\tilde{M}_{4}^{o}\right)^{2} \log \frac{\tilde{M}_{2}^{+^{2}}+\tilde{M}_{4}^{o^{2}}}{2 \mu_{o}^{2}} \\
& \left.+\frac{1}{2}\left(\tilde{M}_{3}^{o}-\tilde{M}_{4}^{o}\right)^{2} \log \frac{\tilde{M}_{3}^{o^{2}}+\tilde{M}_{4}^{o^{2}}}{2 \mu_{o}^{2}}\right\} \\
& U_{\tilde{\chi}}=\frac{4}{3 s_{W}{ }^{2}} \log \frac{\tilde{M}_{2}^{o^{2}}+\tilde{M}_{1}^{+^{2}}}{2 \tilde{M}_{1}^{+^{2}}} \\
& +\frac{1}{3 s_{W}{ }^{2}} \log \left[\frac{\left(\tilde{M}_{3}^{o^{2}}+\tilde{M}_{2}^{+^{2}}\right)\left(\tilde{M}_{4}^{o^{2}}+\tilde{M}_{2}^{+^{2}}\right)}{2 \tilde{M}_{2}^{+^{2}}\left(\tilde{M}_{3}^{o^{2}}+\tilde{M}_{4}^{o^{2}}\right)}\right] \text {, }
\end{aligned}
$$

where the expressions (110) and (111) are valid considering together the above two conditions and also:

$$
\left|M_{i}^{+^{2}}-M_{j}^{o^{2}}\right| \ll\left|M_{i}^{+^{2}}+M_{j}^{o^{2}}\right|, \quad i=1,2 ; \quad j=1,2,3,4 .
$$

Here we have used the values of the coupling matrices $O_{L, R}, O^{\prime}{ }_{L, R}, O^{\prime \prime}{ }_{L, R}$ corresponding to the large neutralinos and charginos masses limit that are given in eqs (41). Notice that the above expressions are valid for both $\mu \geq 0$ and $\mu<0$.

\subsection{Discussion and comments}

- The above expressions for $S, T$ and $U$ are general and depend just on the physical sparticle masses and the generic coefficients $c_{f}, s_{f}\left(f=t, b, \tau, \ldots\right.$ and $0 \leq c_{f}, s_{f}$ $\leq 1)$. The results for these parameters of the various sectors are finite as they must be. The cancellation of divergences occur between the $\tilde{t}$ and $\tilde{b}$ contributions of each generation of squarks, between the $\tilde{\nu}$ and $\tilde{\tau}$ contributions of each generation of sleptons and between the charginos and neutralinos.

- The corrections to these formulae are always suppressed by extra factors of the type

$$
\left[\frac{\tilde{m}_{1}^{2}-\tilde{m}_{2}^{2}}{\tilde{m}_{1}^{2}+\tilde{m}_{2}^{2}}\right]^{n}
$$

which are forced to be small under our assumption $\left|\tilde{m}_{1}^{2}-\tilde{m}_{2}^{2}\right| \ll\left|\tilde{m}_{1}^{2}+\tilde{m}_{2}^{2}\right|$ for the various types of sparticles, and vanish in the infinite masses limit.

- Although the three parameters do not require the same set of conditions on the sparticle masses, the physical and realistic situation corresponds to have fixed all the SUSY spectra at once, and therefore all these conditions must hold together. Thus, by considering:

$$
\begin{aligned}
&\left|\tilde{m}_{t_{1}}^{2}-\tilde{m}_{t_{2}}^{2}\right| \ll\left|\tilde{m}_{t_{1}}^{2}+\tilde{m}_{t_{2}}^{2}\right|, \\
&\left|\tilde{m}_{b_{1}}^{2}-\tilde{m}_{b_{2}}^{2}\right| \ll\left|\tilde{m}_{b_{1}}^{2}+\tilde{m}_{b_{2}}^{2}\right| \quad \text { and } \\
&\left|\tilde{m}_{t_{i}}^{2}-\tilde{m}_{b_{j}}^{2}\right| \ll\left|\tilde{m}_{t_{i}}^{2}+\tilde{m}_{b_{j}}^{2}\right|, \quad(i, j=1,2),
\end{aligned}
$$

is equivalent to say that all the squarks of the same generation have masses of similar large size. Similarly, in the sleptons sector the conditions are:

$$
\begin{aligned}
\left|\tilde{m}_{\nu}^{2}-\tilde{m}_{\tau_{i}}^{2}\right| & \ll\left|\tilde{m}_{\nu}^{2}+\tilde{m}_{\tau_{i}}^{2}\right|(i=1,2) \text { and } \\
\left|\tilde{m}_{\tau_{1}}^{2}-\tilde{m}_{\tau_{2}}^{2}\right| & \ll\left|\tilde{m}_{\tau_{1}}^{2}-\tilde{m}_{\tau_{2}}^{2}\right|,
\end{aligned}
$$

and imply that the sleptons of the same generation have also large masses of similar size. The conditions on the charginos and the neutralinos sector are,

$$
\begin{aligned}
\left|M_{1}^{+^{2}}-M_{2}^{+^{2}}\right| & \ll\left|M_{1}^{+^{2}}+M_{2}^{+^{2}}\right|, \\
\left|M_{i}^{0^{2}}-M_{j}^{0^{2}}\right| & \ll\left|M_{i}^{0^{2}}+M_{j}^{0^{2}}\right| ;(i, j=1,2,3,4), \\
\left|M_{i}^{+^{2}}-M_{j}^{o^{2}}\right| & \ll\left|M_{i}^{+^{2}}+M_{j}^{o^{2}}\right|(i=1,2 ; j=1,2,3,4),
\end{aligned}
$$

and imply analogously that all the large masses $M_{i}^{+}$ and $M_{j}^{0}$ are comparable.

Let us now comment on how the decoupling occurs in the various sectors.

Interestingly, in the squarks sector and by looking just at the $S_{\tilde{q}}$ parameter, there is apparently no decoupling since the dominant contribution goes as:

$$
S_{\tilde{q}} \rightarrow-\sum_{\tilde{q}} \frac{N_{c}}{36 \pi} \log \frac{\tilde{m}_{t_{1}}^{2}}{\tilde{m}_{b_{1}}^{2}},\left(\tilde{m}_{q_{i}}^{2} \gg k^{2}\right)
$$

which under the corresponding conditions $\left|\tilde{m}_{t_{1}}^{2}-\tilde{m}_{t_{2}}^{2}\right| \ll$ $\left|\tilde{m}_{t_{1}}^{2}+\tilde{m}_{t_{2}}^{2}\right|$ and $\left|\tilde{m}_{b_{1}}^{2}-\tilde{m}_{b_{2}}^{2}\right| \ll\left|\tilde{m}_{b_{1}}^{2}+\tilde{m}_{b_{2}}^{2}\right|$ does not vanish 
in the infinite $\tilde{m}_{t_{1}}$ and $\tilde{m}_{b_{1}}$ limit. However, when the three parameters $S_{\tilde{q}}, T_{\tilde{q}}$ and $U_{\tilde{q}}$ are analized together and all the conditions, including the third one, $\left|\tilde{m}_{t_{i}}^{2}-\tilde{m}_{b_{j}}^{2}\right| \ll$ $\left|\tilde{m}_{t_{i}}^{2}+\tilde{m}_{b_{j}}^{2}\right|,(i, j=1,2)$, are required together, then the above dominant term in $S_{\tilde{q}}$ also vanishes in the infinite squark masses limit as it was expected.

In order to show the decoupling explicitly one can go a step further and make an expansion of $S_{\tilde{q}}, T_{\tilde{q}}$ and $U_{\tilde{q}}$ in powers of the proper dimensionless quantities which accordingly must vanish in the considered infinite mass limit. More explicitly, we define the proper expansion parameters in the (third generation) squarks sector as:

$$
\frac{\tilde{m}_{t_{1}}^{2}-\tilde{m}_{b_{1}}^{2}}{\tilde{m}_{t_{1}}^{2}+\tilde{m}_{b_{1}}^{2}}, \frac{\tilde{m}_{b_{1}}^{2}-\tilde{m}_{b_{2}}^{2}}{\tilde{m}_{b_{1}}^{2}+\tilde{m}_{b_{2}}^{2}}, \frac{\tilde{m}_{t_{i}}^{2}-\tilde{m}_{b_{j}}^{2}}{\tilde{m}_{t_{i}}^{2}+\tilde{m}_{b_{j}}^{2}},(i, j=1,2) .
$$

In terms of these parameters we get the following dominant terms in the power expansions of $S_{\tilde{q}}, T_{\tilde{q}}$ and $U_{\tilde{q}}$ :

$$
\begin{aligned}
S_{\tilde{q}} \rightarrow & -\sum_{\tilde{q}} \frac{N_{c}}{18 \pi}\left\{\left(\frac{\tilde{m}_{t_{1}}^{2}-\tilde{m}_{b_{1}}^{2}}{\tilde{m}_{t_{1}}^{2}+\tilde{m}_{b_{1}}^{2}}\right)+s_{b}^{2}\left(\frac{\tilde{m}_{b_{1}}^{2}-\tilde{m}_{b_{2}}^{2}}{\tilde{m}_{b_{1}}^{2}+\tilde{m}_{b_{2}}^{2}}\right)\right. \\
& -s_{t}^{2}\left(\frac{\tilde{m}_{t_{1}}^{2}-\tilde{m}_{t_{2}}^{2}}{\tilde{m}_{t_{1}}^{2}+\tilde{m}_{t_{2}}^{2}}\right)-3 c_{t}^{2} s_{t}^{2}\left(\frac{\tilde{m}_{t_{1}}^{2}-\tilde{m}_{t_{2}}^{2}}{\tilde{m}_{t_{1}}^{2}+\tilde{m}_{t_{2}}^{2}}\right)^{2} \\
& \left.-3 c_{b}^{2} s_{b}^{2}\left(\frac{\tilde{m}_{b_{1}}^{2}-\tilde{m}_{b_{2}}^{2}}{\tilde{m}_{b_{1}}^{2}+\tilde{m}_{b_{2}}^{2}}\right)^{2}\right\}+O\left(\frac{\tilde{m}_{i}^{2}-\tilde{m}_{j}^{2}}{\tilde{m}_{i}^{2}+\tilde{m}_{j}^{2}}\right)^{3},
\end{aligned}
$$

$$
\begin{aligned}
T_{\tilde{q}} \rightarrow & \sum_{\tilde{q}} \frac{N_{c}}{16 \pi} \frac{1}{s_{W}^{2} m_{W}^{2}}\left\{c_{t}^{2} c_{b}^{2}\left(\tilde{m}_{t_{1}}^{2}-\tilde{m}_{b_{1}}^{2}\right)\left(\frac{\tilde{m}_{t_{1}}^{2}-\tilde{m}_{b_{1}}^{2}}{\tilde{m}_{t_{1}}^{2}+\tilde{m}_{b_{1}}^{2}}\right)\right. \\
& +c_{t}^{2} s_{b}^{2}\left(\tilde{m}_{t_{1}}^{2}-\tilde{m}_{b_{2}}^{2}\right)\left(\frac{\tilde{m}_{t_{1}}^{2}-\tilde{m}_{b_{2}}^{2}}{\tilde{m}_{t_{1}}^{2}+\tilde{m}_{b_{2}}^{2}}\right) \\
& -c_{t}^{2} s_{t}^{2}\left(\tilde{m}_{t_{1}}^{2}-\tilde{m}_{t_{2}}^{2}\right)\left(\frac{\tilde{m}_{t_{1}}^{2}-\tilde{m}_{t_{2}}^{2}}{\tilde{m}_{t_{1}}^{2}+\tilde{m}_{t_{2}}^{2}}\right) \\
& +s_{t}^{2} c_{b}^{2}\left(\tilde{m}_{t_{2}}^{2}-\tilde{m}_{b_{1}}^{2}\right)\left(\frac{\tilde{m}_{t_{2}}^{2}-\tilde{m}_{b_{1}}^{2}}{\tilde{m}_{t_{2}}^{2}+\tilde{m}_{b_{1}}^{2}}\right) \\
& +s_{t}^{2} s_{b}^{2}\left(\tilde{m}_{t_{2}}^{2}-\tilde{m}_{b_{2}}^{2}\right)\left(\frac{\tilde{m}_{t_{2}}^{2}-\tilde{m}_{b_{2}}^{2}}{\tilde{m}_{t_{2}}^{2}+\tilde{m}_{b_{2}}^{2}}\right) \\
& \left.-s_{b}^{2} c_{b}^{2}\left(\tilde{m}_{b_{1}}^{2}-\tilde{m}_{b_{2}}^{2}\right)\left(\frac{\tilde{m}_{b_{1}}^{2}-\tilde{m}_{b_{2}}^{2}}{\tilde{m}_{b_{1}}^{2}+\tilde{m}_{b_{2}}^{2}}\right)\right\} \\
& +O\left[\left(\frac{\tilde{m}_{i}^{2}-\tilde{m}_{j}^{2}}{m_{W}^{2}}\right)\left(\frac{\tilde{m}_{i}^{2}-\tilde{m}_{j}^{2}}{\tilde{m}_{i}^{2}+\tilde{m}_{j}^{2}}\right)^{2}\right], \\
& -c_{t}^{2} s_{b}^{2}\left(\frac{\tilde{m}_{t_{1}}^{2}-\tilde{m}_{b_{2}}^{2}}{\tilde{m}_{t_{1}}^{2}+\tilde{m}_{b_{2}}^{2}}\right)^{2}-s_{t}^{2} s_{b}^{2}\left(\frac{\tilde{m}_{t_{2}}^{2}-\tilde{m}_{b_{2}}^{2}}{\tilde{m}_{t_{2}}^{2}+\tilde{m}_{b_{2}}^{2}}\right)^{2} \\
& \sum \frac{N_{c}}{12 \pi}\left\{-c_{t}^{2} c_{b}^{2}\left(\frac{\tilde{m}_{t_{1}}^{2}-\tilde{m}_{b_{1}}^{2}}{\tilde{m}_{t_{1}}^{2}+\tilde{m}_{b_{1}}^{2}}\right)^{2}\right. \\
& {[114) }
\end{aligned}
$$

$$
\begin{aligned}
& -s_{t}^{2} c_{b}^{2}\left(\frac{\tilde{m}_{t_{2}}^{2}-\tilde{m}_{b_{1}}^{2}}{\tilde{m}_{t_{2}}^{2}+\tilde{m}_{b_{1}}^{2}}\right)^{2}+c_{t}^{2} s_{t}^{2}\left(\frac{\tilde{m}_{t_{1}}^{2}-\tilde{m}_{t_{2}}^{2}}{\tilde{m}_{t_{1}}^{2}+\tilde{m}_{t_{2}}^{2}}\right)^{2} \\
& \left.+s_{b}^{2} c_{b}^{2}\left(\frac{\tilde{m}_{b_{1}}^{2}-\tilde{m}_{b_{2}}^{2}}{\tilde{m}_{b_{1}}^{2}+\tilde{m}_{b_{2}}^{2}}\right)^{2}\right\}+O\left(\frac{\tilde{m}_{i}^{2}-\tilde{m}_{j}^{2}}{\tilde{m}_{i}^{2}+\tilde{m}_{j}^{2}}\right)^{4} .
\end{aligned}
$$

First, we see that in the limit of exact custodial $S U(2)_{V}$ symmetry, which corresponds to $\tilde{m}_{t_{1}}=\tilde{m}_{b_{1}} \equiv \tilde{m}_{1}, \tilde{m}_{t_{2}}=$ $\tilde{m}_{b_{2}} \equiv \tilde{m}_{2}$ and $c_{t}=c_{b} \equiv c, s_{t}=s_{b} \equiv s$, both $T_{\tilde{q}}$ and $U_{\tilde{q}}$ vanish as it is expected, whereas $S_{\tilde{q}}$ goes as,

$$
S_{\tilde{q}} \rightarrow \sum_{\tilde{q}} \frac{N_{c}}{3 \pi} c^{2} s^{2}\left(\frac{\tilde{m}_{1}^{2}-\tilde{m}_{2}^{2}}{\tilde{m}_{1}^{2}+\tilde{m}_{2}^{2}}\right)^{2}+O\left(\frac{\tilde{m}_{1}^{2}-\tilde{m}_{2}^{2}}{\tilde{m}_{1}^{2}+\tilde{m}_{2}^{2}}\right)^{4}
$$

Second, the above formulae show that the decoupling indeed occurs in the three parameters since they go to zero as some power of the parameters

$$
\left[\left(\tilde{m}_{i}^{2}-\tilde{m}_{j}^{2}\right) /\left(\tilde{m}_{i}^{2}+\tilde{m}_{j}^{2}\right)\right]
$$

which vanish in the infinite masses limit, $\tilde{m}_{i}^{2}, \tilde{m}_{j}^{2} \rightarrow \infty$, with $\left|\tilde{m}_{i}^{2}-\tilde{m}_{j}^{2}\right| \ll\left|\tilde{m}_{i}^{2}+\tilde{m}_{j}^{2}\right|$. Besides, the decoupling is much faster in $U_{\tilde{q}}$ than in $S_{\tilde{q}}$ and $T_{\tilde{q}}$. These results confirm the numerical analyses performed in the literature and agree with the qualitative behaviour discussed in [6-8].

However, we would like to emphasize once more that, contrary to most of the studies in the literature (with the exception of those on $\Delta \rho$ ), our results for $S, T$ and $U$ in this section are model independent and do not make any reference on whether there is or not a common effective scale of supersymmetry breaking. We have neither assumed here the common assumption for the MSSM masses (see Sect. 3) that $\left(\tilde{m}_{i}^{2}-\tilde{m}_{j}^{2}\right) \sim O\left(m_{z}^{2}\right)$ or $\sim O\left(m_{f}^{2}\right)$. Generically speaking, these mass differences could well be larger than the gauge boson or fermion masses and therefore the rapidity of decoupling can vary from one SUSY breaking model to another. In particular by comparing $S_{\tilde{q}}$ and $T_{\tilde{q}}$ in the non-custodial symmetric case, we see that their dominant contributions go respectively as

$$
\left(\frac{\tilde{m}_{i}^{2}-\tilde{m}_{j}^{2}}{\tilde{m}_{i}^{2}+\tilde{m}_{j}^{2}}\right) \text { and }\left(\frac{\tilde{m}_{i}^{2}-\tilde{m}_{j}^{2}}{m_{W}^{2}}\right)\left(\frac{\tilde{m}_{i}^{2}-\tilde{m}_{j}^{2}}{\tilde{m}_{i}^{2}+\tilde{m}_{j}^{2}}\right) .
$$

Therefore the later could be enhanced (suppressed) respect to the first one if

$$
\left(\tilde{m}_{i}^{2}-\tilde{m}_{j}^{2}\right) \gg m_{W}{ }^{2} \quad\left(\left(\tilde{m}_{i}^{2}-\tilde{m}_{j}^{2}\right) \ll m_{W}{ }^{2}\right) .
$$

In summary, from our previous analysis we can infer with complete generality that the largest contributions from the squarks sector to $S_{\tilde{q}}, T_{\tilde{q}}$ and $U_{\tilde{q}}$ come from the squarks pairs with the largest $\left[\left(\tilde{m}_{i}^{2}-\tilde{m}_{j}^{2}\right) /\left(\tilde{m}_{i}^{2}+\tilde{m}_{j}^{2}\right)\right]$ values.

Parallel results for the sleptons sector can be obtained by making the following replacements in the above formulae: $\tilde{q} \rightarrow \tilde{l}, N_{c} \rightarrow 1, \tilde{m}_{t_{1}} \rightarrow \tilde{m}_{\nu}, \tilde{m}_{b_{1}} \rightarrow \tilde{m}_{\tau_{1}}, \tilde{m}_{b_{2}} \rightarrow$ $\tilde{m}_{\tau_{2}}, c_{t} \rightarrow 1, s_{t} \rightarrow 0, c_{b} \rightarrow c_{\tau}$ and $s_{b} \rightarrow s_{\tau}$. 
With regard to the neutralinos and charginos sector and by looking at $(109$ - 111) we first notice that, in the large masses limit, the first chargino $\tilde{\chi}_{1}^{+}$and the two first neutralinos $\tilde{\chi}_{1}^{o}$ and $\tilde{\chi}_{2}^{o}$ decouple completely in the $S$ parameter. These are precisely the chargino and neutralinos, that in the large masses limit become predominantly gauginos. The decoupling of the other eigenstates $\tilde{\chi}_{2}^{+}, \tilde{\chi}_{3}^{o}$ and $\tilde{\chi}_{4}^{o}$ in $S$ is not evident at a first sight, since it depends on the relative size of the $\tilde{\chi}_{2}^{+}$mass with respect to the masses of the neutralinos $\tilde{\chi}_{3}^{o}$ and $\tilde{\chi}_{4}^{o}$. However, we have seen in the second section, that in the large masses limit, their corresponding squared mass eigenvalues approach to a common value $\mu^{2}$ and, in consequence, the decoupling in $S_{\tilde{\chi}}$ does finally occur. Notice that this result is not model dependent either, since this common value $\mu^{2}$ is the unique squared mass parameter that is allowed by supersymmetry to be present at the Lagrangian level and does not depend on the particular assumed SUSY breaking mechanism. Similarly, in the $T_{\tilde{\chi}}$ and $U_{\tilde{\chi}}$ parameters the decoupling occurs exactly if the mass eigenvalues in the large mass limit are considered, i.e, $\tilde{M}_{1}^{+^{2}} \rightarrow M_{2}^{2}, \tilde{M}_{2}^{+^{2}} \rightarrow$ $\mu^{2}, \tilde{M}_{1}^{o^{2}} \rightarrow M_{1}^{2}, \tilde{M}_{2}^{o^{2}} \rightarrow M_{2}^{2}$ and $\tilde{M}_{3}^{o^{2}}=\tilde{M}_{4}^{o^{2}} \rightarrow \mu^{2}$.

Notice that the discussion on how the decoupling occurs in the particular case of the MSSM with soft SUSY breaking terms is evident from the above analisis together with the arguments given in Sect.3. In conclusion, the decoupling of sparticles in $S, T$ and $U$ in our asymptotic limit takes place.

Finally, we discuss in the rest of this section the alternative possibility for the squarks sector of the MSSM:

$$
\left|\frac{\tilde{m}_{t_{1}}^{2}-\tilde{m}_{t_{2}}^{2}}{\tilde{m}_{t_{1}}^{2}+\tilde{m}_{t_{2}}^{2}}\right| \sim O(1), \quad\left|\frac{\tilde{m}_{b_{1}}^{2}-\tilde{m}_{b_{2}}^{2}}{\tilde{m}_{b_{1}}^{2}+\tilde{m}_{b_{2}}^{2}}\right| \sim O(1),
$$

which has been considered in detail in Sect. 3. As we have explained there, this case implies that $s_{t}$ and $s_{b}$ go to zero in the asymptotic limit of $M_{S U S Y} \rightarrow \infty$. From the exact results of $S, T$ and $U$ parameters for the squarks sector which can be taken, for instance, from (B.3), (B.6) and (B.9) of [6], and by taking the limit $s_{t}, s_{b} \rightarrow 0$ we obtain:

$$
\begin{aligned}
S & \longrightarrow-\frac{N_{c}}{36 \pi} \log \frac{\tilde{m}_{t_{1}}^{2}}{\tilde{m}_{b_{1}}^{2}}, \\
T & \longrightarrow \frac{N_{c}}{16 \pi} \frac{1}{s_{W}^{2} m_{W}^{2}} g\left(\tilde{m}_{t_{1}}, \tilde{m}_{b_{1}}\right), \\
U & \longrightarrow \frac{N_{c}}{12 \pi} f\left(\tilde{m}_{t_{1}}, \tilde{m}_{b_{1}}\right),
\end{aligned}
$$

where,

$$
\begin{aligned}
g\left(m_{1}, m_{2}\right) & \equiv m_{1}^{2}+m_{2}^{2}-2 \frac{m_{1}^{2} m_{2}^{2}}{m_{1}^{2}-m_{2}^{2}} \log \frac{m_{1}^{2}}{m_{2}^{2}} \\
f\left(m_{1}, m_{2}\right) & \equiv-\frac{5}{3}+\frac{4 m_{1}^{2} m_{2}^{2}}{\left(m_{1}^{2}-m_{2}^{2}\right)^{2}} \\
& +\frac{\left(m_{1}^{2}+m_{2}^{2}\right)\left(m_{1}^{4}+m_{2}^{4}-4 m_{1}^{2} m_{2}^{2}\right)}{\left(m_{1}^{2}-m_{2}^{2}\right)^{3}} \log \frac{m_{1}^{2}}{m_{2}^{2}}
\end{aligned}
$$

with $g\left(m_{1}, m_{2}\right) \approx 0$ and $f\left(m_{1}, m_{2}\right) \approx 0$ if $m_{1} \approx m_{2}$.
Notice that only squarks $\tilde{t}_{1}$ and $\tilde{b}_{1}$ remain in the above expressions and they are precisely the squarks whose masses in the large masses limit do always get close, namely $\tilde{m}_{t_{1}}^{2} \approx M_{\tilde{Q}}^{2}$ and $\tilde{m}_{b_{1}}^{2} \approx M_{\tilde{Q}}^{2}$.

From the above expressions and by following the discussion presented in Sect. 3 we conclude that the three parameters also vanish in this case as it was expected. It completes our proof of decoupling of sparticles in these observables.

\section{Conclusions}

Althought there are indications that the common assumption of decoupling of heavy supersymmetric particles in the MSSM leading to the SM as the remaining low energy effective theory is correct a formal proof is still lacking.

This formal proof should be performed along the lines stated in the Decoupling Theorem and by means of the powerful techniques of the Effective Field Theories. The computation of the effective action for the standard particles which results by integrating out all the heavy supersymmetric particles will provide the answer to this question. If the contribution from the heavy sparticles to the effective action can be absorbed into redefinitions of the Standard Model parameters or they are suppressed by inverse powers of the heavy sparticles masses, then the decoupling will be demonstrated.

In this paper we have computed the two-point functions part of this effective action for the electroweak gauge bosons, $W^{ \pm}, Z$ and $\gamma$, that results by integrating out the squarks, sleptons, charginos and neutralinos to one loop level.

We have analyzed carefully the large SUSY masses limit of these two-point Green functions and we have presented analytical results for them as well as for the selfenergies and the $R^{X Y}$ functions, which are valid in that limit. These formulae are given in terms of the sparticle masses and, therefore, they are general. Namely, they do not depend on the particular choice for the soft-breaking terms. In our opinion, it is more convenient for the analysis of the phenomenon of decoupling to use the physical sparticle masses themselves, being the proper parameters, rather than some other possible mass parameters of the MSSM as, for instance, the $\mu$-parameter or the soft-SUSY breaking parameters.

The results for the two-point functions of the electroweak gauge bosons indicate that there is indeed decoupling of squarks, sleptons, gauginos and neutralinos in the limit where the sparticle masses are all large as compared to the $W^{ \pm}$and $Z$ masses and the external momentum. In taking the large mass limit we have not assumed exact universality of the masses but we have always worked under the plausible assumption that the differences of their squared masses are much smaller than their sums. As can be seen from the formulae of Appendix C, all the remaining contributions to the two point functions from sparticles can be absorbed into redefinitions of the Standard Model parameters $m_{Z}, m_{W}$ and $e$ and the gauge bosons wave functions. The contributions which are not shown 
in our formulae all vanish in the asymptotic limit of very large sparticle masses.

We have shown that the decoupling of sparticles also takes place in the $S, T$ and $U$ parameters, and we have presented explicit formulae for these parameters, which illustrate analytically how this decoupling occurs.

Finally, we have explored to what extent the hypothesis of generation of SUSY masses by soft-SUSY breaking terms is relevant for decoupling and we have found instead that the requirement of $S U(3)_{\mathrm{c}} \times S U(2)_{\mathrm{L}} \times U(1)_{\mathrm{Y}}$ gauge invariance of the explicit mass terms by itself is sufficient to get it.

Acknowledgements. We are indebted to A.Casas for his advising in theoretical aspects of the MSSM and for interesting discussions. We thank L.Diaz-Cruz for participating in the very early stages of this work. M.J.H wishes to acknowledge D.Espriu, H.Haber, W.Hollik and J.Solà for interesting discussions on the subject of decoupling. A.D thanks the CERN-TH division for his kind hospitality during the last part of this work. This work has been partially supported by the Spanish Ministerio de Educación y Ciencia under projects CICYT AEN96-1664 and AEN93-0776, and the fellowship AP95 00503301

\section{Appendix A}

In this appendix, we present the exact results of the selfenergies $\Sigma^{X Y}(k)$ and the $R^{X Y}(k)$ functions of (95) to one loop.

Self-energies $\Sigma^{X Y}(k)$ :

$$
\begin{aligned}
& \Sigma^{A A}(k)=i e^{2} \sum_{\widetilde{f}}\left\{2 \sum_{a} I_{0}\left(\tilde{m}_{f_{a}}^{2}\right)\left(\widehat{Q}_{f}^{2}\right)_{a a}\right. \\
& \left.\quad-\sum_{a, b}\left(\widehat{Q}_{f}\right)_{a b}\left(\widehat{Q}_{f}\right)_{b a} A_{f}^{a b}\left(k, \tilde{m}_{f_{a}}, \tilde{m}_{f_{b}}\right)\right\} \\
& +2 i e^{2} \sum_{i=1}^{2}\left\{C^{i i}\left(k, \tilde{M}_{i}^{+}, \tilde{M}_{i}^{+}\right)+2 \hat{I}^{i i}\left(k, \tilde{M}_{i}^{+}, \tilde{M}_{i}^{+}\right)\right\}
\end{aligned}
$$

$$
\begin{aligned}
& \Sigma^{Z Z}(k)=\frac{i g^{2}}{c_{W}{ }^{2}} \sum_{\widetilde{f}}\left\{2 \sum_{a} I_{0}\left(\tilde{m}_{f_{a}}^{2}\right)\left(\widehat{G}_{f}^{2}\right)_{a a}\right. \\
& \left.\quad-\sum_{a, b}\left(\widehat{G}_{f}\right)_{a b}\left(\widehat{G}_{f}\right)_{b a} A_{f}^{a b}\left(k, \tilde{m}_{f_{a}}, \tilde{m}_{f_{b}}\right)\right\} \\
& \quad+\frac{i}{2} \frac{g^{2}}{c_{W}{ }^{2}} \sum_{i, j=1}^{4}\left\{\left(O_{L}^{\prime \prime i j} O_{L}^{\prime \prime j i}+O_{R}^{\prime \prime i j} O_{R}^{\prime \prime j} i\right) C^{i j}\left(k, \tilde{M}_{i}^{0}, \tilde{M}_{j}^{0}\right)\right. \\
& \left.+2\left(O_{L}^{\prime \prime i j} O_{R}^{\prime \prime j i}+O_{R}^{\prime \prime i j} O_{L}^{\prime \prime j i}\right) \hat{I}^{i j}\left(k, \tilde{M}_{i}^{0}, \tilde{M}_{j}^{0}\right)\right\}
\end{aligned}
$$

$$
\begin{aligned}
& +i \frac{g^{2}}{c_{W}{ }^{2}} \sum_{i, j=1}^{2}\left\{\left(O_{L}^{\prime i j} O_{L}^{\prime j i}+O_{R}^{\prime i j} O_{R}^{\prime j i}\right) C^{i j}\left(k, \tilde{M}_{i}^{+}, \tilde{M}_{j}^{+}\right)\right. \\
& \left.+2\left(O_{L}^{\prime i j} O_{R}^{\prime j i}+O_{R}^{\prime i j} O_{L}^{\prime j i}\right) \hat{I}^{i j}\left(k, \tilde{M}_{i}^{+}, \tilde{M}_{j}^{+}\right)\right\} \quad(\mathrm{A} 2) \\
& \Sigma^{A z}(k)=\frac{i g e}{c_{W}} \sum_{\tilde{f}}\left\{2 \sum_{a} I_{0}\left(\tilde{m}_{f_{a}}^{2}\right)\left(\widehat{Q}_{f} \widehat{G}_{f}\right)_{a a}\right. \\
& \left.-\sum_{a, b}\left(\widehat{Q}_{f}\right)_{a b}\left(\widehat{G}_{f}\right)_{b a} A_{f}^{a b}\left(k, \tilde{m}_{f_{a}}, \tilde{m}_{f_{b}}\right)\right\} \\
& -\frac{i g e}{c_{W}} \sum_{i=1}^{2}\left\{( O _ { L } ^ { \prime i i } + O _ { R } ^ { \prime i } ) \left(C^{i i}\left(k, \tilde{M}_{i}^{+}, \tilde{M}_{i}^{+}\right)\right.\right. \\
& \left.\left.+2 \hat{I}^{i i}\left(k, \tilde{M}_{i}^{+}, \tilde{M}_{i}^{+}\right)\right)\right\} \\
& \left.+2\left(O_{L}^{i j} O_{R}^{+j i}+O_{R}^{i j} O_{L}^{+j i}\right) \hat{I}^{i j}\left(k, \tilde{M}_{i}^{0}, \tilde{M}_{j}^{+}\right)\right\} \\
& +i g^{2} \sum_{i=1}^{4} \sum_{j=1}^{2}\left\{\left(O_{L}^{i j} O_{L}^{+j i}+O_{R}^{i j} O_{R}^{+j i}\right) C^{i j}\left(k, \tilde{M}_{i}^{0}, \tilde{M}_{j}^{+}\right)\right. \\
& \left.-\sum_{a, b}\left(\Sigma_{f}^{t b}\right)_{a b}\left(\Sigma_{f}^{t b}\right)_{a b} A_{f}^{a b}\left(k, \tilde{m}_{f_{a}}, \tilde{m}_{f_{b}}\right)\right\} \\
& \Sigma^{i}{ }^{2} \sum_{\tilde{f}}\left\{\sum_{a}\left(\Sigma_{f}\right)_{a a} I_{0}\left(\tilde{m}_{f_{a}}^{2}\right)\right.
\end{aligned}
$$

\section{$R^{X Y}(k)$ functions:}

$$
\begin{aligned}
& R^{A A}(k)=-i e^{2} \sum_{\widetilde{f}} \sum_{a, b}\left(\widehat{Q}_{f}\right)_{a b}\left(\widehat{Q}_{f}\right)_{b a} B_{f}^{a b}\left(k, \tilde{m}_{f_{a}}, \tilde{m}_{f_{b}}\right) \\
& +2 i e^{2} \sum_{i=1}^{2} D^{i i}\left(k, \tilde{M}_{i}^{+}, \tilde{M}_{i}^{+}\right) \\
& R^{Z z}(k)=-\frac{i g^{2}}{c_{W}{ }^{2}} \sum_{\widetilde{f}} \sum_{a, b}\left(\widehat{G}_{f}\right)_{a b}\left(\widehat{G}_{f}\right)_{b a} \\
& \times B_{f}^{a b}\left(k, \tilde{m}_{f_{a}}, \tilde{m}_{f_{b}}\right) \\
& +\frac{i}{2} \frac{g^{2}}{c_{W}{ }^{2}} \sum_{i, j=1}^{4}\left(O_{L}^{\prime \prime i j} O_{L}^{\prime \prime j i}+O_{R}^{\prime \prime i j} O_{R}^{\prime \prime j i}\right) D^{i j}\left(k, \tilde{M}_{i}^{0}, \tilde{M}_{j}^{0}\right) \\
& +i \frac{g^{2}}{c_{W}{ }^{2}} \sum_{i, j=1}^{2}\left({O^{\prime}}_{L}^{i j} O_{L}^{\prime j i}+O_{R}^{\prime i j} O_{R}^{\prime j i}\right) D^{i j}\left(k, \tilde{M}_{i}^{+}, \tilde{M}_{j}^{+}\right)
\end{aligned}
$$

$$
\begin{gathered}
R^{A Z}(k)=-\frac{i g e}{c_{W}} \sum_{\widetilde{f}} \sum_{a, b}\left(\widehat{Q}_{f}\right)_{a b}\left(\widehat{G}_{f}\right)_{b a} B_{f}^{a b}\left(k, \tilde{m}_{f_{a}}, \tilde{m}_{f_{b}}\right) \\
-\frac{i g e}{c_{W}} \sum_{i=1}^{2}\left({O^{\prime}}_{L}^{i i}+O^{\prime i i}\right) D^{i i}\left(k, \tilde{M}_{i}^{+}, \tilde{M}_{i}^{+}\right)
\end{gathered}
$$




$$
\begin{gathered}
R^{W W}(k)=-\frac{i g^{2}}{2} \sum_{\widetilde{f}} \sum_{a, b}\left(\Sigma_{f}^{t b}\right)_{a b}\left(\Sigma_{f}^{t b}\right)_{a b} B_{f}^{a b}\left(k, \tilde{m}_{f_{a}}, \tilde{m}_{f_{b}}\right) \\
+i g^{2} \sum_{i=1}^{4} \sum_{j=1}^{2}\left(O_{L}^{i j} O_{L}^{+j i}+O_{R}^{i j} O_{R}^{+j i}\right) D^{i j}\left(k, \tilde{M}_{i}^{0}, \tilde{M}_{j}^{+}\right)
\end{gathered}
$$

The functions $A_{f}^{a b}, B_{f}^{a b}, C^{i j}, D^{i j}$ and $\hat{I}^{i j}$ have been defined in (97) and (94) respectively.

\section{Appendix B}

In this appendix, we give the results of the one loop integrals of $((88)-(94))$ in the large masses limit and include an example of the large mass techniques used in the calculation.

We start by giving the results of the integrals. Here and from now on,

$$
\Delta_{\epsilon}=\frac{2}{\epsilon}-\gamma_{\epsilon}+\log (4 \pi), \quad \epsilon=4-D,
$$

and $\mu_{o}$ is the usual mass scale of dimensional regularization.

The integrals are given by:

$$
I_{0}\left(\tilde{m}_{f a}^{2}\right)=\frac{i}{16 \pi^{2}}\left(\Delta_{\epsilon}+1-\log \frac{\tilde{m}_{f a}^{2}}{\mu_{o}^{2}}\right) \tilde{m}_{f a}^{2}
$$

This is a well known result in dimensional regularization and is exact for all $\tilde{m}_{f a}^{2}$.

- If $\tilde{m}_{f a}^{2}, \tilde{m}_{f b}^{2} \gg k^{2}$ and $\left|\tilde{m}_{f a}^{2}-\tilde{m}_{f b}^{2}\right| \ll\left|\tilde{m}_{f a}^{2}+\tilde{m}_{f b}^{2}\right|:$

$$
\begin{aligned}
A_{f}^{a b}\left(k, \tilde{m}_{f_{a}}, \tilde{m}_{f_{b}}\right)= & \frac{i}{16 \pi^{2}}\left\{\left(\tilde{m}_{f a}^{2}+\tilde{m}_{f b}^{2}\right)\right. \\
& \times\left(\Delta_{\epsilon}+1-\log \frac{\tilde{m}_{f a}^{2}+\tilde{m}_{f b}^{2}}{2 \mu_{o}^{2}}\right) \\
& \left.-\frac{1}{3} k^{2}\left(\Delta_{\epsilon}-\log \frac{\tilde{m}_{f a}^{2}+\tilde{m}_{f b}^{2}}{2 \mu_{o}^{2}}\right)\right\} \\
B_{f}^{a b}\left(k, \tilde{m}_{f_{a}}, \tilde{m}_{f_{b}}\right)= & \frac{i}{16 \pi^{2}} \frac{1}{3}\left(\Delta_{\epsilon}-\log \frac{\tilde{m}_{f a}^{2}+\tilde{m}_{f b}^{2}}{2 \mu_{o}^{2}}\right)
\end{aligned}
$$

- If $\tilde{M}_{i}^{2}, \tilde{M}_{j}^{2} \gg k^{2}$ and $\left|\tilde{M}_{i}^{2}-\tilde{M}_{j}^{2}\right| \ll\left|\tilde{M}_{i}^{2}+\tilde{M}_{j}^{2}\right|$ :

$$
\begin{aligned}
I^{i j}\left(k, \tilde{M}_{i}, \tilde{M}_{j}\right)= & \frac{i}{16 \pi^{2}}\left(\Delta_{\epsilon}-\log \frac{\tilde{M}_{i}^{2}+\tilde{M}_{j}^{2}}{2 \mu_{o}^{2}}\right), \\
I_{\mu \nu}^{i j}\left(k, \tilde{M}_{i}, \tilde{M}_{j}\right)= & \frac{i}{16 \pi^{2}}\left\{\frac{1}{4}\left(\tilde{M}_{i}^{2}+\tilde{M}_{j}^{2}\right) g_{\mu \nu}\right. \\
& \times\left(\Delta_{\epsilon}+1-\log \frac{\tilde{M}_{i}^{2}+\tilde{M}_{j}^{2}}{2 \mu_{o}^{2}}\right) \\
& -\frac{k^{2}}{12} g_{\mu \nu}\left(\Delta_{\epsilon}-\log \frac{\tilde{M}_{i}^{2}+\tilde{M}_{j}^{2}}{2 \mu_{o}^{2}}\right)
\end{aligned}
$$

$$
\begin{aligned}
& \left.+\frac{1}{3} k_{\mu} k_{\nu}\left(\Delta_{\epsilon}-\log \frac{\tilde{M}_{i}^{2}+\tilde{M}_{j}^{2}}{2 \mu_{o}^{2}}\right)\right\}, \\
I_{\mu \nu}^{i j}\left(k, \tilde{M}_{i}, \tilde{M}_{j}\right)= & -\frac{i}{16 \pi^{2}} \frac{k_{\mu} k_{\nu}}{2}\left(\Delta_{\epsilon}-\log \frac{\tilde{M}_{i}^{2}+\tilde{M}_{j}^{2}}{2 \mu_{o}^{2}}\right), \\
C^{i j}\left(k, \tilde{M}_{i}, \tilde{M}_{j}\right)= & \frac{i}{16 \pi^{2}}\left\{-\left(\tilde{M}_{i}^{2}+\tilde{M}_{j}^{2}\right)\right. \\
& \times\left(\Delta_{\epsilon}-\log \frac{\tilde{M}_{i}^{2}+\tilde{M}_{j}^{2}}{2 \mu_{o}^{2}}\right) \\
& \left.+\frac{2}{3} k^{2}\left(\Delta_{\epsilon}-\frac{1}{2}-\log \frac{\tilde{M}_{i}^{2}+\tilde{M}_{j}^{2}}{2 \mu_{o}^{2}}\right)\right\}, \\
D^{i j}\left(k, \tilde{M}_{i}, \tilde{M}_{j}\right)= & -\frac{i}{16 \pi^{2}} \frac{2}{3}\left(\Delta_{\epsilon}-\log \frac{\tilde{M}_{i}^{2}+\tilde{M}_{j}^{2}}{2 \mu_{o}^{2}}\right), \\
\hat{I}^{i j}\left(k, \tilde{M}_{i}, \tilde{M}_{j}\right)= & \frac{i}{16 \pi^{2}} \frac{1}{2}\left\{\frac{1}{3} k^{2}\right. \\
& +\left(\tilde{M}_{i}^{2}+\tilde{M}_{j}^{2}\right)\left(\Delta_{\epsilon}-\log \frac{\tilde{M}_{i}^{2}+\tilde{M}_{j}^{2}}{2 \mu_{o}^{2}}\right) \\
& \left.-\left(\tilde{M}_{i}-\tilde{M}_{j}\right)^{2}\left(\Delta_{\epsilon}-\log \frac{\tilde{M}_{i}^{2}+\tilde{M}_{j}^{2}}{2 \mu_{o}^{2}}\right)\right\} .
\end{aligned}
$$

The corrections to these formulas are suppressed by inverse powers of $\left(\tilde{m}_{f a}^{2}+\tilde{m}_{f b}^{2}\right)$ or $\left(\tilde{M}_{i}^{2}+\tilde{M}_{j}^{2}\right)$ respectively and vanish in the infinite masses limit. For the calculation of these integrals we have used the m-Theorem [23].

\section{m-Theorem:}

Consider the integral,

$$
I(p, m)=m^{\beta} \int d^{4} k \frac{M(k)}{\prod_{i}\left(l_{i}^{2}+m_{i}^{2}\right)^{n_{i}}}
$$

where $l_{i}=k+\sum_{i=1}^{E} b_{i j} p_{j}$, being $p_{j}$ the external momenta which lay in a bounded subdomain of $\Re^{4}, m_{i}=0$ or $\mathrm{m}$, $\beta$ is an arbitrary real number and $M(k)$ is a monomial in the components of $k$.

If:

the integral $I(p, m)$ is both UV and IR convergent by power counting at non-exceptional external momenta and if

$$
d-w<0,
$$

being $d$ the mass dimension of $I(p, m)$ and $w$ the minimum of zero and the infrared divergence degree of $I(p, m)$ at zero external momenta, then:

$I(p, m)$ goes to zero when $m$ goes to infinity. 
As an example of the applications of this theorem let us consider the following integral:

$$
I_{\mu \nu}(k, m)=\int d \widehat{q} \frac{q_{\mu} q_{\nu}}{\left[(k+q)^{2}-m^{2}\right]\left[q^{2}-m^{2}\right]}
$$

The superficial degree of UV divergence of $I_{\mu \nu}$ is 2 at $D=4$. The first step is to rearrange algebraically the integrand by using:

$$
\frac{1}{\left[(k+q)^{2}-m^{2}\right]}=\frac{1}{\left[q^{2}-m^{2}\right]}-\frac{2 q k+k^{2}}{\left[(k+q)^{2}-m^{2}\right]\left[q^{2}-m^{2}\right]}
$$

in order to lower the degree of UV divergence of the integral corresponding to the second term in (B6). This algebraic rearrangement must be applied as many times as necessary until obtaining a convergent integral which satisfies the requirements of the $\mathrm{m}$-Theorem.

By substituting (B6) in (B5), one gets:

$$
\begin{aligned}
I_{\mu \nu}(k, m)= & \int d \widehat{q} \frac{q_{\mu} q_{\nu}}{\left[q^{2}-m^{2}\right]^{2}} \\
& -\int d \widehat{q} \frac{(2 q k) q_{\mu} q_{\nu}}{\left[(k+q)^{2}-m^{2}\right]\left[q^{2}-m^{2}\right]^{2}} \\
& -\int d \widehat{q} \frac{k^{2} q_{\mu} q_{\nu}}{\left[(k+q)^{2}-m^{2}\right]\left[q^{2}-m^{2}\right]^{2}} \\
\equiv & M_{\mu \nu}-N_{\mu \nu}-k^{2} P_{\mu \nu}
\end{aligned}
$$

where $M_{\mu \nu}$ can be evaluated exactly using the standard techniques of dimensional regularization.

Let us concentrate in the last term $P_{\mu \nu}$ that is logarithmically divergent. By using again (B6) one gets,

$$
\begin{aligned}
P_{\mu \nu}= & \int d \widehat{q} \frac{q_{\mu} q_{\nu}}{\left[q^{2}-m^{2}\right]^{3}} \\
& -\int d \widehat{q} \frac{(2 q k) q_{\mu} q_{\nu}}{\left[(k+q)^{2}-m^{2}\right]\left[q^{2}-m^{2}\right]^{3}} \\
& -\int d \widehat{q} \frac{k^{2} q_{\mu} q_{\nu}}{\left[(k+q)^{2}-m^{2}\right]\left[q^{2}-m^{2}\right]^{3}}
\end{aligned}
$$

Now, the last two integrals are convergent at $D=4$ and satisfy the requirements of the m-Theorem. Therefore, these two terms go to zero when $D \rightarrow 4$ and $m \rightarrow \infty$. The first term can be evaluated exactly using the standard techniques obtaining:

$$
P_{\mu \nu}=\left(\frac{i}{16 \pi^{2}}\right) \frac{1}{4} g_{\mu \nu}\left(\Delta_{\epsilon}-\log \frac{m^{2}}{\mu_{o}^{2}}\right)
$$

Finally, by using the same procedure to compute $N_{\mu \nu}$, one gets:

$$
\begin{aligned}
I_{\mu \nu}(k, m)= & \frac{i}{16 \pi^{2}}\left\{\frac{1}{2} g_{\mu \nu}\left(\Delta_{\epsilon}+1-\log \frac{m^{2}}{\mu_{o}^{2}}\right) m^{2}\right. \\
& \left.+\left(\frac{1}{3} k_{\mu} k_{\nu}-\frac{k^{2}}{12} g_{\mu \nu}\right)\left(\Delta_{\epsilon}-\log \frac{m^{2}}{\mu_{o}^{2}}\right)\right\}
\end{aligned}
$$

Another example that we want to show due to the more intricate manipulations is $\hat{I}^{i j}$ of (94). In this case we define two new parameters that do not appear in the other integrals, $\overline{\mathrm{m}}^{2} \equiv\left(\tilde{M}_{i}-\tilde{M}_{j}\right)^{2}$ and $\overline{\mathrm{M}}^{2} \equiv\left(\tilde{M}_{i}+\tilde{M}_{j}\right)^{2}$. In terms of these and the other more commom parameters, $\hat{\mathrm{M}}^{2}$ and $\hat{\mathrm{m}}^{2}, \hat{I}^{i j}$ can be written as:

$$
\hat{I}^{i j}=\frac{1}{4} \int d \widehat{q} \frac{\overline{\mathrm{M}}^{2}-\overline{\mathrm{m}}^{2}}{\left[q^{2}-\hat{\mathrm{M}}^{2}+\hat{\mathrm{m}}^{2}\right]\left[(k+q)^{2}-\hat{\mathrm{M}}^{2}-\hat{\mathrm{m}}^{2}\right]}
$$

where $\hat{\mathrm{M}}^{2} \equiv \frac{1}{2}\left(\tilde{M}_{i}^{2}+\tilde{M}_{j}^{2}\right)$ and $\hat{\mathrm{m}}^{2} \equiv \frac{1}{2}\left(\tilde{M}_{i}^{2}-\tilde{M}_{j}^{2}\right)$.

By using the relations between all parameters, $\overline{\mathrm{m}}^{2}, \overline{\mathrm{M}}^{2}$, $\hat{\mathrm{m}}^{2}, \hat{\mathrm{M}}^{2}$, the above integral can be written as:

$$
\hat{I}^{i j}=I_{1}-\frac{1}{2} I_{2}
$$

being,

$$
\begin{aligned}
& I_{1}=\int d \widehat{q} \frac{\hat{\mathrm{M}}^{2}}{\left[q^{2}-\hat{\mathrm{M}}^{2}+\hat{\mathrm{m}}^{2}\right]\left[(k+q)^{2}-\hat{\mathrm{M}}^{2}-\hat{\mathrm{m}}^{2}\right]}, \\
& I_{2}=\int d \widehat{q} \frac{\overline{\mathrm{m}}^{2}}{\left[q^{2}-\hat{\mathrm{M}}^{2}+\hat{\mathrm{m}}^{2}\right]\left[(k+q)^{2}-\hat{\mathrm{M}}^{2}-\hat{\mathrm{m}}^{2}\right]} .
\end{aligned}
$$

The first step is again to rearrange algebraically the integrand as many times as necessary until obtaining a convergent integral. The appropriate algebraic manipulations in this case are:

$$
\begin{aligned}
& \frac{1}{\left[q^{2}-\hat{\mathrm{M}}^{2} \pm \hat{\mathrm{m}}^{2}\right]}= \frac{1}{\left[q^{2}-\hat{\mathrm{M}}^{2}\right]} \\
& \mp \frac{\hat{\mathrm{m}}^{2}}{\left[q^{2}-\hat{\mathrm{M}}^{2} \pm \hat{\mathrm{m}}^{2}\right]\left[q^{2}-\hat{\mathrm{M}}^{2}\right]} \\
& \frac{1}{\left[(k+q)^{2}-\hat{\mathrm{M}}^{2}-\hat{\mathrm{m}}^{2}\right]}=\frac{1}{\left[q^{2}-\hat{\mathrm{M}}^{2}-\hat{\mathrm{m}}^{2}\right]} \\
&-\frac{2 q k}{\left[(k+q)^{2}-\hat{\mathrm{M}}^{2}-\hat{\mathrm{m}}^{2}\right]\left[q^{2}-\hat{\mathrm{M}}^{2}-\hat{\mathrm{m}}^{2}\right]}
\end{aligned}
$$

In particular for $I_{2}$, by using (B13) twice, (B14) once and by applying the $\mathrm{m}$-Theorem to the convergent integrals we get:

$$
I_{2}=\frac{i}{16 \pi^{2}} \overline{\mathrm{m}}^{2}\left(\Delta_{\epsilon}-\log \frac{\hat{\mathrm{M}}^{2}}{\mu_{o}^{2}}\right)
$$

In order to compute $I_{1}$, notice that it can be written as:

$$
\begin{aligned}
I_{1}= & -\int d \widehat{q} \frac{1}{\left[(k+q)^{2}-\hat{\mathrm{M}}^{2}-\hat{\mathrm{m}}^{2}\right]} \\
& +\int d \widehat{q} \frac{q^{2}+\hat{\mathrm{m}}^{2}}{\left[q^{2}-\hat{\mathrm{M}}^{2}+\hat{\mathrm{m}}^{2}\right]\left[(k+q)^{2}-\hat{\mathrm{M}}^{2}-\hat{\mathrm{m}}^{2}\right]} \\
= & -I_{1}^{\prime}+I_{1}^{\prime \prime} .
\end{aligned}
$$


By doing the change of variables $(k+q) \rightarrow q$ and by considering (B13) twice, the first integral of (B16) can be written as:

$$
\begin{aligned}
I_{1}^{\prime}= & \int d \widehat{q} \frac{1}{\left[q^{2}-\hat{\mathrm{M}}^{2}\right]}+\int d \widehat{q} \frac{\hat{\mathrm{m}}^{2}}{\left[q^{2}-\hat{\mathrm{M}}^{2}\right]^{2}} \\
& +\int d \widehat{q} \frac{\hat{\mathrm{m}}^{4}}{\left[q^{2}-\hat{\mathrm{M}}^{2}\right]^{2}\left[q^{2}-\hat{\mathrm{M}}^{2}-\hat{\mathrm{m}}^{2}\right]}
\end{aligned}
$$

where the first two terms can be again evaluated exactly by using the standard techniques and the last one goes to zero by virtue of the $\mathrm{m}$-Theorem.

Similarly, to compute the second part, $I_{1}^{\prime \prime}$, we consider (B13) and (B14) as many times as necessary and apply the $\mathrm{m}$-Theorem. We obtain:

$$
I_{1}=\frac{i}{16 \pi^{2}}\left\{\hat{\mathrm{M}}^{2}\left(\Delta_{\epsilon}-\log \frac{\hat{\mathrm{M}}^{2}}{\mu_{o}^{2}}\right)+\frac{k^{2}}{6}\right\} .
$$

Finally, by collecting all the results we get,

$$
\begin{aligned}
& \hat{I}^{i j}\left(k, \tilde{M}_{i}, \tilde{M}_{j}\right)=\frac{i}{16 \pi^{2}} \frac{1}{2}\left\{\frac{1}{3} k^{2}\right. \\
& +\left(\tilde{M}_{i}^{2}+\tilde{M}_{j}^{2}\right)\left(\Delta_{\epsilon}-\log \frac{\tilde{M}_{i}^{2}+\tilde{M}_{j}^{2}}{2 \mu_{o}^{2}}\right) \\
& \left.-\left(\tilde{M}_{i}-\tilde{M}_{j}\right)^{2}\left(\Delta_{\epsilon}-\log \frac{\tilde{M}_{i}^{2}+\tilde{M}_{j}^{2}}{2 \mu_{o}^{2}}\right)\right\} .
\end{aligned}
$$

\section{Appendix C}

In this appendix we present the asymptotic results in the large sparticle masses limit for the two-point functions $\Sigma_{T}^{X Y}$ and $\Sigma_{L}^{X Y}$ of $(96)$.

$$
\begin{aligned}
& \Sigma_{T}^{A A}(k)=\Sigma_{T}^{A A}(k)_{\tilde{q}}+\Sigma_{T}^{A A}(k)_{\tilde{l}}+\Sigma_{T}^{A A}(k)_{\tilde{\chi}}+\Sigma_{T}^{A A}(k)_{H} \\
& \Sigma_{L}^{A A}(k)=\Sigma_{L}^{A A}(k)_{\tilde{q}}+\Sigma_{L}^{A A}(k)_{\tilde{l}}+\Sigma_{L}^{A A}(k)_{\tilde{\chi}}+\Sigma_{L}^{A A}(k)_{H}
\end{aligned}
$$

- If $\quad \tilde{m}_{t_{1}}^{2}, \tilde{m}_{t_{2}}^{2}, \tilde{m}_{b_{1}}^{2}, \tilde{m}_{b_{2}}^{2} \gg k^{2}$ :

$$
\begin{aligned}
\Sigma_{T}^{A A}(k)_{\tilde{q}}= & -N_{c} \frac{e^{2}}{16 \pi^{2}} \frac{1}{27} k^{2} \\
& \times \sum_{\tilde{q}}\left\{4\left(2 \Delta_{\epsilon}-\log \frac{\tilde{m}_{t_{1}}^{2}}{\mu_{o}^{2}}-\log \frac{\tilde{m}_{t_{2}}^{2}}{\mu_{o}^{2}}\right)\right. \\
& \left.+\left(2 \Delta_{\epsilon}-\log \frac{\tilde{m}_{b_{1}}^{2}}{\mu_{o}^{2}}-\log \frac{\tilde{m}_{b_{2}}^{2}}{\mu_{o}^{2}}\right)\right\},
\end{aligned}
$$

- If $\quad \tilde{m}_{\tau_{1}}^{2}, \tilde{m}_{\tau_{2}}^{2} \gg k^{2}$ :

$$
\Sigma_{T}^{A A}(k)_{\tilde{l}}=-\frac{e^{2}}{16 \pi^{2}} \frac{k^{2}}{3} \sum_{\tilde{l}}\left(2 \Delta_{\epsilon}-\log \frac{\tilde{m}_{\tau_{1}}^{2}}{\mu_{o}^{2}}-\log \frac{\tilde{m}_{\tau_{2}}^{2}}{\mu_{o}^{2}}\right)
$$

- If $\quad \tilde{M}_{1}^{+2}, \tilde{M}_{2}^{+2} \gg k^{2}$ :

$$
\Sigma_{T}^{A A}(k)_{\tilde{\chi}}=-\frac{e^{2}}{16 \pi^{2}} \frac{4}{3} k^{2}\left(2 \Delta_{\epsilon}-\log \frac{\tilde{M}_{1}^{+^{2}}}{\mu_{o}^{2}}-\log \frac{\tilde{M}_{2}^{+^{2}}}{\mu_{o}^{2}}\right),
$$

and for the longitudinal parts, we get:

$$
\Sigma_{L}^{A A}(k)_{\tilde{q}}=\Sigma_{L}^{A A}(k)_{\tilde{l}}=\Sigma_{L}^{A A}(k)_{\tilde{\chi}}=0
$$

$$
\Sigma_{T}^{A Z}(k)=\Sigma_{T}^{A Z}(k)_{\tilde{q}}+\Sigma_{T}^{A Z}(k)_{\tilde{l}}+\Sigma_{T}^{A Z}(k)_{\tilde{\chi}}+\Sigma_{T}^{A Z}(k)_{H}
$$$$
\Sigma_{L}^{A Z}(k)=\Sigma_{L}^{A Z}(k)_{\tilde{q}}+\Sigma_{L}^{A Z}(k)_{\tilde{l}}+\Sigma_{L}^{A Z}(k)_{\tilde{\chi}}+\Sigma_{L}^{A Z}(k)_{H}
$$

- If $\tilde{m}_{t_{1}}^{2}, \tilde{m}_{t_{2}}^{2}, \tilde{m}_{b_{1}}^{2}, \tilde{m}_{b_{2}}^{2} \gg k^{2}:$

$$
\begin{aligned}
\sum_{T}^{A Z}(k)_{\tilde{q}}= & -N_{c} \frac{e^{2}}{16 \pi^{2}} \frac{1}{9 s_{W} c_{W}} k^{2} \\
& \times \sum_{\tilde{q}}\left\{2\left(\frac{1}{2} c_{t}^{2}-2 s_{W}^{2}\right)\left(\Delta_{\epsilon}-\log \frac{\tilde{m}_{t_{1}}^{2}}{\mu_{o}^{2}}\right)\right. \\
& +2\left(\frac{1}{2} s_{t}^{2}-2 s_{W}^{2}\right)\left(\Delta_{\epsilon}-\log \frac{\tilde{m}_{t_{2}}^{2}}{\mu_{o}^{2}}\right) \\
& -\left(-\frac{1}{2} c_{b}^{2}+\frac{1}{3} s_{W}^{2}\right)\left(\Delta_{\epsilon}-\log \frac{\tilde{m}_{b_{1}}^{2}}{\mu_{o}^{2}}\right) \\
& \left.-\left(-\frac{1}{2} s_{b}^{2}+\frac{1}{3} s_{W}^{2}\right)\left(\Delta_{\epsilon}-\log \frac{\tilde{m}_{b_{2}}^{2}}{\mu_{o}^{2}}\right)\right\},
\end{aligned}
$$

- If $\tilde{m}_{\tau_{1}}^{2}, \tilde{m}_{\tau_{2}}^{2} \gg k^{2}$ :

$$
\begin{aligned}
\Sigma_{T}^{A Z}(k)_{\tilde{l}}= & -\frac{e^{2}}{16 \pi^{2}} \frac{1}{3 s_{W} c_{W}} k^{2} \\
& \times \sum_{\tilde{l}}\left\{\left(\frac{1}{2} c_{\tau}^{2}-s_{W}^{2}\right)\left(\Delta_{\epsilon}-\log \frac{\tilde{m}_{\tau_{1}}^{2}}{\mu_{o}^{2}}\right)\right. \\
& \left.+\left(\frac{1}{2} s_{\tau}^{2}-s_{W}^{2}\right)\left(\Delta_{\epsilon}-\log \frac{\tilde{m}_{\tau_{2}}^{2}}{\mu_{o}^{2}}\right)\right\},
\end{aligned}
$$

- If $\quad \tilde{M}_{1}^{+2}, \tilde{M}_{2}^{+2} \gg k^{2}$ :

$$
\begin{aligned}
\Sigma_{T}^{A Z}(k)_{\tilde{\chi}}= & \frac{e^{2}}{16 \pi^{2}} \frac{k^{2}}{s_{W} c_{W}} \frac{4}{3}\left\{\left(s_{W}{ }^{2}-1\right)\left(\Delta_{\epsilon}-\log \frac{\tilde{M}_{1}^{+2}}{\mu_{o}^{2}}\right)\right. \\
& \left.+\left(s_{W}{ }^{2}-\frac{1}{2}\right)\left(\Delta_{\epsilon}-\log \frac{\tilde{M}_{2}^{+2}}{\mu_{o}^{2}}\right)\right\}, \quad(\mathrm{C} 9)
\end{aligned}
$$

and for the longitudinal parts, we get:

$$
\Sigma_{L}^{A Z}(k)_{\tilde{q}}=\Sigma_{L}^{A Z}(k)_{\tilde{l}}=\Sigma_{L}^{A Z}(k)_{\tilde{\chi}}=0
$$

$$
\begin{aligned}
& \Sigma_{T}^{Z Z}(k)=\Sigma_{T}^{Z Z}(k)_{\tilde{q}}+\Sigma_{T}^{Z Z}(k)_{\tilde{l}}+\Sigma_{T}^{Z Z}(k)_{\tilde{\chi}}+\Sigma_{T}^{Z Z}(k)_{H} \\
& \Sigma_{L}^{Z Z}(k)=\Sigma_{L}^{Z Z}(k)_{\tilde{q}}+\Sigma_{L}^{Z Z}(k)_{\tilde{l}}+\Sigma_{L}^{Z Z}(k)_{\tilde{\chi}}+\Sigma_{L}^{Z Z}(k)_{H}
\end{aligned}
$$


- If $\quad \tilde{m}_{t_{1}}^{2}, \tilde{m}_{t_{2}}^{2}, \tilde{m}_{b_{1}}^{2}, \tilde{m}_{b_{2}}^{2} \gg k^{2}$;

$\left|\tilde{m}_{t_{1}}^{2}-\tilde{m}_{t_{2}}^{2}\right| \ll\left|\tilde{m}_{t_{1}}^{2}+\tilde{m}_{t_{2}}^{2}\right|$

$\left|\tilde{m}_{b_{1}}^{2}-\tilde{m}_{b_{2}}^{2}\right| \ll\left|\tilde{m}_{b_{1}}^{2}+\tilde{m}_{b_{2}}^{2}\right|:$

$\Sigma_{T}^{Z Z}(k)_{\tilde{q}}=N_{c} \frac{e^{2}}{16 \pi^{2}} \frac{1}{s_{W}^{2} c_{W}^{2}}$

$\times \sum_{\tilde{q}}\left\{\frac{1}{2}\left[c_{t}^{2} s_{t}^{2} h\left(\tilde{m}_{t_{1}}^{2}, \tilde{m}_{t_{2}}^{2}\right)+c_{b}^{2} s_{b}^{2} h\left(\tilde{m}_{b_{1}}^{2}, \tilde{m}_{b_{2}}^{2}\right)\right]\right.$

$-\frac{1}{3} k^{2}\left[\left(\frac{c_{t}^{2}}{2}-\frac{2 s_{W}^{2}}{3}\right)^{2}\left(\Delta_{\epsilon}-\log \frac{\tilde{m}_{t_{1}}^{2}}{\mu_{o}^{2}}\right)\right.$

$+\left(\frac{s_{t}^{2}}{2}-\frac{2 s_{W}^{2}}{3}\right)^{2}\left(\Delta_{\epsilon}-\log \frac{\tilde{m}_{t_{2}}^{2}}{\mu_{o}^{2}}\right)$

$+\left(-\frac{c_{b}^{2}}{2}+\frac{s_{W}^{2}}{3}\right)^{2}\left(\Delta_{\epsilon}-\log \frac{\tilde{m}_{b_{1}}^{2}}{\mu_{o}^{2}}\right)$

$+\left(-\frac{s_{b}^{2}}{2}+\frac{s_{W}^{2}}{3}\right)^{2}\left(\Delta_{\epsilon}-\log \frac{\tilde{m}_{b_{2}}^{2}}{\mu_{o}^{2}}\right)$

$+\frac{1}{2} s_{t}^{2} c_{t}^{2}\left(\Delta_{\epsilon}-\log \frac{\tilde{m}_{t_{1}}^{2}+\tilde{m}_{t_{2}}^{2}}{2 \mu_{o}^{2}}\right)$

$\left.\left.+\frac{1}{2} s_{b}^{2} c_{b}^{2}\left(\Delta_{\epsilon}-\log \frac{\tilde{m}_{b_{1}}^{2}+\tilde{m}_{b_{2}}^{2}}{2 \mu_{o}^{2}}\right)\right]\right\}$,

$\Sigma_{L}^{Z Z}(k)_{\tilde{q}}=N_{c} \frac{e^{2}}{16 \pi^{2}} \frac{1}{2 s_{W}^{2} c_{W}^{2}}$

$\times \sum_{\tilde{q}}\left\{c_{t}^{2} s_{t}^{2} h\left(\tilde{m}_{t_{1}}^{2}, \tilde{m}_{t_{2}}^{2}\right)+c_{b}^{2} s_{b}^{2} h\left(\tilde{m}_{b_{1}}^{2}, \tilde{m}_{b_{2}}^{2}\right)\right\}$,

- If $\quad \tilde{m}_{\nu}^{2}, \tilde{m}_{\tau_{1}}^{2}, \tilde{m}_{\tau_{2}}^{2} \gg k^{2}$

$\left|\tilde{m}_{\tau_{1}}^{2}-\tilde{m}_{\tau_{2}}^{2}\right| \ll\left|\tilde{m}_{\tau_{1}}^{2}+\tilde{m}_{\tau_{2}}^{2}\right|:$

$\Sigma_{T}^{Z Z}(k)_{\tilde{l}}=-\frac{e^{2}}{16 \pi^{2}} \frac{1}{s_{W}^{2} c_{W}^{2}} \sum_{\tilde{l}}\left\{-\frac{1}{2} c_{\tau}^{2} s_{\tau}^{2} h\left(\tilde{m}_{\tau_{1}}^{2}, \tilde{m}_{\tau_{2}}^{2}\right)\right.$

$+\frac{1}{3} k^{2}\left[\frac{1}{4}\left(\Delta_{\epsilon}-\log \frac{\tilde{m}_{\nu}^{2}}{\mu_{o}^{2}}\right)\right.$

$+\left(\frac{-c_{\tau}^{2}}{2}+s_{W}^{2}\right)^{2}\left(\Delta_{\epsilon}-\log \frac{\tilde{m}_{\tau_{1}}^{2}}{\mu_{o}^{2}}\right)$

$+\left(-\frac{s_{\tau}^{2}}{2}+s_{W}^{2}\right)^{2}\left(\Delta_{\epsilon}-\log \frac{\tilde{m}_{\tau_{2}}^{2}}{\mu_{o}^{2}}\right)$

$\left.\left.+\frac{1}{2} s_{\tau}^{2} c_{\tau}^{2}\left(\Delta_{\epsilon}-\log \frac{\tilde{m}_{\tau_{1}}^{2}+\tilde{m}_{\tau_{2}}^{2}}{2 \mu_{o}^{2}}\right)\right]\right\}$,

$$
\Sigma_{L}^{Z Z}(k)_{\tilde{l}}=\frac{e^{2}}{16 \pi^{2}} \frac{1}{s_{W}^{2} c_{W}^{2}} \frac{1}{2} \sum_{\tilde{l}}\left\{c_{\tau}^{2} s_{\tau}^{2} h\left(\tilde{m}_{\tau_{1}}^{2}, \tilde{m}_{\tau_{2}}^{2}\right)\right\}
$$

- If $\tilde{M}_{1,2}^{+2}, \tilde{M}_{j}^{o 2} \gg k^{2}$,

$\left|\tilde{M}_{1}^{+2}-\tilde{M}_{2}^{+2}\right| \ll\left|\tilde{M}_{1}^{+2}+\tilde{M}_{2}^{+2}\right| ;$

$\left|\tilde{M}_{i}^{o 2}-\tilde{M}_{j}^{o 2}\right| \ll\left|\tilde{M}_{i}^{o 2}+\tilde{M}_{j}^{o 2}\right|, i, j=1,2,3,4$ :

$\Sigma_{T}^{Z Z}(k)_{\tilde{\chi}}=-\frac{e^{2}}{16 \pi^{2}} \frac{1}{s_{W}^{2} c_{W}^{2}}\left\{-\frac{1}{2}\left(\tilde{M}_{3}^{o}-\tilde{M}_{4}^{o}\right)^{2}\right.$

$$
\begin{aligned}
& \times\left(\Delta_{\epsilon}-\log \frac{\tilde{M}_{3}^{o 2}+\tilde{M}_{4}^{o 2}}{2 \mu_{o}^{2}}\right) \\
& +\frac{1}{3} k^{2}\left[4\left(s_{W}^{2}-1\right)^{2}\left(\Delta_{\epsilon}-\log \frac{\tilde{M}_{1}^{+2}}{\mu_{o}^{2}}\right)\right. \\
& +4\left(s_{W}^{2}-\frac{1}{2}\right)^{2}\left(\Delta_{\epsilon}-\log \frac{\tilde{M}_{2}^{+2}}{\mu_{o}^{2}}\right) \\
& \left.\left.+\left(\Delta_{\epsilon}-\log \frac{\tilde{M}_{3}^{o 2}+\tilde{M}_{4}^{o 2}}{2 \mu_{o}^{2}}\right)\right]\right\}, \\
\Sigma_{L}^{Z Z}(k)_{\tilde{\chi}}= & \frac{e^{2}}{16 \pi^{2}} \frac{1}{2 s_{W}^{2} c_{W}^{2}}\left\{\left(\tilde{M}_{3}^{o}-\tilde{M}_{4}^{o}\right)^{2}\right. \\
& \left.\times\left(\Delta_{\epsilon}-\log \frac{\tilde{M}_{3}^{o 2}+\tilde{M}_{4}^{o 2}}{2 \mu_{o}^{2}}\right)\right\},
\end{aligned}
$$

where $h\left(m_{1}^{2}, m_{2}^{2}\right)$ of $(\mathrm{C} 12-\mathrm{C} 15)$ has been defined in (105).

$$
\begin{aligned}
\Sigma_{T}^{W W}(k)= & \Sigma_{T}^{W W}(k)_{\tilde{q}}+\Sigma_{T}^{W W}(k)_{\tilde{l}} \\
& +\Sigma_{T}^{W W}(k)_{\tilde{\chi}}+\Sigma_{T}^{W W}(k)_{H} \\
\Sigma_{L}^{W W}(k)= & \Sigma_{L}^{W W}(k)_{\tilde{q}}+\Sigma_{L}^{W W}(k)_{\tilde{l}} \\
& +\Sigma_{L}^{W W}(k)_{\tilde{\chi}}+\Sigma_{L}^{W W}(k)_{H}
\end{aligned}
$$

- If $\tilde{m}_{t_{1}}^{2}, \tilde{m}_{t_{2}}^{2}, \tilde{m}_{b_{1}}^{2}, \tilde{m}_{b_{2}}^{2} \gg k^{2}$;

$\left|\tilde{m}_{t_{i}}^{2}-\tilde{m}_{b_{j}}^{2}\right| \ll\left|\tilde{m}_{t_{i}}^{2}+\tilde{m}_{b_{j}}^{2}\right|, i, j=1,2:$

$\Sigma_{T}^{W W}(k)_{\tilde{q}}=N_{c} \frac{e^{2}}{16 \pi^{2}} \frac{1}{2 s_{W}^{2}} \sum_{\tilde{q}}\left\{\left[c_{t}^{2} c_{b}^{2} h\left(\tilde{m}_{t_{1}}^{2}, \tilde{m}_{b_{1}}^{2}\right)\right.\right.$

$$
+c_{t}^{2} s_{b}^{2} h\left(\tilde{m}_{t_{1}}^{2}, \tilde{m}_{b_{2}}^{2}\right)+s_{t}^{2} c_{b}^{2} h\left(\tilde{m}_{t_{2}}^{2}, \tilde{m}_{b_{1}}^{2}\right)
$$

$\left.+s_{t}^{2} s_{b}^{2} h\left(\tilde{m}_{t_{2}}^{2}, \tilde{m}_{b_{2}}^{2}\right)\right]$

$-\frac{1}{3} k^{2}\left[c_{t}^{2} c_{b}^{2}\left(\Delta_{\epsilon}-\log \frac{\tilde{m}_{t_{1}}^{2}+\tilde{m}_{b_{1}}^{2}}{2 \mu_{o}^{2}}\right)\right.$

$+c_{t}^{2} s_{b}^{2}\left(\Delta_{\epsilon}-\log \frac{\tilde{m}_{t_{1}}^{2}+\tilde{m}_{b_{2}}^{2}}{2 \mu_{o}^{2}}\right)$

$+s_{t}^{2} c_{b}^{2}\left(\Delta_{\epsilon}-\log \frac{\tilde{m}_{t_{2}}^{2}+\tilde{m}_{b_{1}}^{2}}{2 \mu_{o}^{2}}\right)$

$\left.\left.+s_{t}^{2} s_{b}^{2}\left(\Delta_{\epsilon}-\log \frac{\tilde{m}_{t_{2}}^{2}+\tilde{m}_{b_{2}}^{2}}{2 \mu_{o}^{2}}\right)\right]\right\}$,

$\Sigma_{L}^{W W}(k)_{\tilde{q}}=N_{c} \frac{e^{2}}{16 \pi^{2}} \frac{1}{2 s_{W}^{2}} \sum_{\tilde{q}}\left\{c_{t}^{2} c_{b}^{2} h\left(\tilde{m}_{t_{1}}^{2}, \tilde{m}_{b_{1}}^{2}\right)\right.$ $+c_{t}^{2} s_{b}^{2} h\left(\tilde{m}_{t_{1}}^{2}, \tilde{m}_{b_{2}}^{2}\right)+s_{t}^{2} c_{b}^{2} h\left(\tilde{m}_{t_{2}}^{2}, \tilde{m}_{b_{1}}^{2}\right)$

$\left.+s_{t}^{2} s_{b}^{2} h\left(\tilde{m}_{t_{2}}^{2}, \tilde{m}_{b_{2}}^{2}\right)\right\}$,

- If $\tilde{m}_{\nu}^{2}, \tilde{m}_{\tau_{1}}^{2}, \tilde{m}_{\tau_{2}}^{2} \gg k^{2}$,

$\left|\tilde{m}_{\nu}^{2}-\tilde{m}_{\tau_{i}}^{2}\right| \ll\left|\tilde{m}_{\nu}^{2}+\tilde{m}_{\tau_{i}}^{2}\right|, i=1,2:$

$\Sigma_{T}^{W W}(k)_{\tilde{l}}=\frac{e^{2}}{16 \pi^{2}} \frac{1}{2 s_{W}^{2}} \sum_{\tilde{l}}\left\{c_{\tau}^{2} h\left(\tilde{m}_{\nu}^{2}, \tilde{m}_{\tau_{1}}^{2}\right)\right.$ 


$$
\begin{aligned}
& +s_{\tau}^{2} h\left(\tilde{m}_{\nu}^{2}, \tilde{m}_{\tau_{2}}^{2}\right) \\
& -\frac{1}{3} k^{2}\left[c_{\tau}^{2}\left(\Delta_{\epsilon}-\log \frac{\tilde{m}_{\nu}^{2}+\tilde{m}_{\tau_{1}}^{2}}{2 \mu_{o}^{2}}\right)\right. \\
& \left.\left.+s_{\tau}^{2}\left(\Delta_{\epsilon}-\log \frac{\tilde{m}_{\nu}^{2}+\tilde{m}_{\tau_{2}}^{2}}{2 \mu_{o}^{2}}\right)\right]\right\} \\
\Sigma_{L}^{W W}(k)_{\tilde{l}}= & \frac{e^{2}}{16 \pi^{2}} \frac{1}{2 s_{W}^{2}} \sum_{\tilde{l}}\left\{c_{\tau}^{2} h\left(\tilde{m}_{\nu}^{2}, \tilde{m}_{\tau_{1}}^{2}\right)\right. \\
& \left.+s_{\tau}^{2} h\left(\tilde{m}_{\nu}^{2}, \tilde{m}_{\tau_{2}}^{2}\right)\right\},
\end{aligned}
$$

- If $\quad \tilde{M}_{i}^{o 2}, \tilde{M}_{j}^{+2} \gg k^{2}$,

$$
\left|\tilde{M}_{i}^{o 2}-\tilde{M}_{j}^{+2}\right| \ll\left|\tilde{M}_{i}^{o 2}+\tilde{M}_{j}^{+2}\right|, i=1,2,3,4:
$$$$
\Sigma_{T}^{W W}(k)_{\tilde{\chi}}=-\frac{e^{2}}{16 \pi^{2}} \frac{1}{s_{W}^{2}}\left\{-2\left(\tilde{M}_{1}^{+}-\tilde{M}_{2}^{o}\right)^{2}\right.
$$$$
\times\left(\Delta_{\epsilon}-\log \frac{\tilde{M}_{1}^{+2}+\tilde{M}_{2}^{o 2}}{2 \mu_{o}^{2}}\right)
$$$$
-\frac{1}{2}\left(\tilde{M}_{2}^{+}-\tilde{M}_{3}^{o}\right)^{2}\left(\Delta_{\epsilon}-\log \frac{\tilde{M}_{2}^{+2}+\tilde{M}_{3}^{o 2}}{2 \mu_{o}^{2}}\right)
$$$$
-\frac{1}{2}\left(\tilde{M}_{2}^{+}-\tilde{M}_{4}^{o}\right)^{2}\left(\Delta_{\epsilon}-\log \frac{\tilde{M}_{2}^{+2}+\tilde{M}_{4}^{o 2}}{2 \mu_{o}^{2}}\right)
$$$$
+\frac{1}{3} k^{2}\left[4\left(\Delta_{\epsilon}-\log \frac{\tilde{M}_{1}^{+2}+\tilde{M}_{2}^{o 2}}{2 \mu_{o}^{2}}\right)\right.
$$$$
+\left(\Delta_{\epsilon}-\log \frac{\tilde{M}_{2}^{+2}+\tilde{M}_{3}^{o 2}}{2 \mu_{o}^{2}}\right)
$$$$
\left.\left.+\left(\Delta_{\epsilon}-\log \frac{\tilde{M}_{2}^{+2}+\tilde{M}_{4}^{o 2}}{2 \mu_{o}^{2}}\right)\right]\right\},
$$

$$
\begin{aligned}
\Sigma_{L}^{W W}(k)_{\tilde{\chi}}= & \frac{e^{2}}{16 \pi^{2}} \frac{1}{s_{W}^{2}}\left\{2\left(\tilde{M}_{1}^{+}-\tilde{M}_{2}^{o}\right)^{2}\right. \\
& \times\left(\Delta_{\epsilon}-\log \frac{\tilde{M}_{1}^{+2}+\tilde{M}_{2}^{o 2}}{2 \mu_{o}^{2}}\right) \\
& +\frac{1}{2}\left(\tilde{M}_{2}^{+}-\tilde{M}_{3}^{o}\right)^{2}\left(\Delta_{\epsilon}-\log \frac{\tilde{M}_{2}^{+2}+\tilde{M}_{3}^{o 2}}{2 \mu_{o}^{2}}\right) \\
& \left.+\frac{1}{2}\left(\tilde{M}_{2}^{+}-\tilde{M}_{4}^{o}\right)^{2}\left(\Delta_{\epsilon}-\log \frac{\tilde{M}_{2}^{+2}+\tilde{M}_{4}^{o 2}}{2 \mu_{o}^{2}}\right)\right\},
\end{aligned}
$$

We also include here the large masses limit of the frequently appearing function $h\left(m_{1}^{2}, m_{2}^{2}\right)$ defined in (105):

- If $\left|m_{1}^{2}-m_{2}^{2}\right| \ll\left|m_{1}^{2}+m_{2}^{2}\right|, m_{1}^{2} ; m_{2}^{2}$ large,

$h\left(m_{1}^{2}, m_{2}^{2}\right) \rightarrow \frac{m_{1}^{2}-m_{2}^{2}}{2}\left[\frac{\left(m_{1}^{2}-m_{2}^{2}\right)}{\left(m_{1}^{2}+m_{2}^{2}\right)}+O\left(\frac{m_{1}^{2}-m_{2}^{2}}{m_{1}^{2}+m_{2}^{2}}\right)^{2}\right]$

\section{References}

1. For an introduction to supersymmetry see, e.g. J. Wess, J. Bagger, Supersymmetry and Supergravity, Princenton University Press, (1983); N.P. Nilles, Phys. Rep. 110, 1 (1984); D. Bailin, A. Love, Supersymmetric Gauge Field Theory and String Theory, Institute of Physics Publishing, (1996)

2. H. Haber, G.L. Kane, Phys. Rep. 117, 75 (1985); J.F. Gunion, H. Haber, Nucl. Phys. B 272, 1 (1986); Nucl. Phys. B 278, 449 (1986)

3. Particle Data Book, Phys. Rev. D 54, 1 (1996)

4. L. Girardello, M.T. Grisaru, Nucl. Phys. B 194, 65 (1982)

5. For a pedagogical introduction to the MSSM see, H. Haber, Introductory low-energy supersymmetry. Published in Boulder TASI-92:589-688, Univ. of Colorado, SCIPP92/93, hep-ph/9306207

6. P.H. Chankowski et al, Nucl. Phys. B 417, 101 (1994)

7. H. Haber, When are radiative corrections important in the Minimal Supersymmetric Model. (UC, Santa Cruz). SCIPP-93-06, Mar 1993. 52pp. Proceedings of INFN Eloisatron Project: 23rd Workshop: The Decay Properties of SUSY Particles, Erice, Italy, 28 Sep-4 Oct 1992. In *Erice 1992, 23rd Eloisatron workshop, Properties of SUSY particles *321-372, hep-ph/9305248

8. D. García, J. Solà, Mod. Phys. Lett. A 9, 211 (1994); D. García, J. Solà, R.A. Jiménez, Phys. Lett. B 347, 309, 321 (1995); D. García, R.A. Jiménez, J. Solà, W. Hollik, Nucl. Phys. B 427, 53 (1994); J.A. Coarasa et al., Eur. Phys. J. C 2, 373 (1998); A. Djouadi et al., Eur. Phys. J. C 1, 149 (1998)

9. T. Inami, C.S. Lim, A. Yamada, Mod. Phys. Lett. A 7, 2789 (1992)

10. For a recent work on the large $\mu$ limit see, J.L. Diaz-Cruz, Phys. Rev. D 56, 523 (1997)

11. H. Haber, Higgs bosons in the Minimal Supersymmetric Model: The influence of radiative corrections. (UC, Santa Cruz). SCIPP-92/31, Published in Perspectives on Higgs Physics, World Scientific Publ., (1992) pp. 79-125

12. M. Carena, M. Quirós, C.E.M. Wagner, Nucl. Phys. B 461, 407 (1996)

13. H. Haber, Non-Minimal Higgs Sectors: The Decoupling Limit and its Phenomenological Implications,. Talk presented at the Workshop on Physics from the Planck Scale to the Electroweak Scale, (SUSY 94), Warsaw, Poland, 2124/Sept. 94, hep-ph/9501320; P. Ciafaloni and D. Espriu, Phys. Rev. D 56, 1752 (1997); A. Djouadi et al., PM/96-39, KA-TP-30-1996, hep-ph/9701342, (Z. Phys. C to appear)

14. T. Appelquist, J. Carazzone, Phys. Rev. D 11, 2856 (1975)

15. M. Bohm, H. Spiesberger, W. Hollik, Fortschr. Phys. 34, 11, 687, 751 (1986)

16. M. Veltman, Act. Phys. Pol. B 8, 475 (1977); Nucl. Phys. B 123, 89 (1977); D.R.T. Jones, M. Veltman, Nucl. Phys. B 19, 146 (1981); M. Chanowitz, M. Furman, I. Hinchliffe, Phys. Lett. B 78, 285 (1978); Nucl. Phys. B 153, 402 (1979)

17. G. Lin, H. Steger, Y. Yao Phys. Rev. D 49, 2414 (1994); F. Feruglio, L. Maiani, A. Masiero, Nucl. Phys. B 387, 523 (1992)

18. T. Appelquist, C. Bernard, Phys. Rev. D 22, 200 (1980); A.C. Longhitano, Nucl. Phys. B 188, 118 (1981); Phys. Rev. D 22, 1166 (1980); M.J. Herrero, E.R. Morales Nucl. Phys. B 418, 431 (1994); Nucl. Phys. B 437, 319 (1995); D. Espriu, J. Matias, Phys. Lett. B 341, 332 (1995); 
S. Dittmaier, C. Grosse-Knetter, Phys. Rev. D 52, 7276 (1995); Nucl. Phys. B 459, 497 (1996)

19. For an introduction to the subject of integration of heavy fields, computation of effective actions see, for instance, A. Dobado et al., Effective Lagrangians for the Standard Model, Springer-Verlag, (1997)

20. L. Ibañez, G.G. Ross, Phys. Lett. B 110, 227 (1982); K. Inoue et al., Prog. Theor. Phys. 67, 1859 (1982); L. AlvarezGaumé, J. Polchinsky, M. Wise, Nucl. Phys. B 221, 495 (1983); J. Ellis et al., Phys. Lett. B 125, 275 (1983); L. Ibañez, C. López, Phys. Lett. B 126, 54 (1983); Nucl. Phys. B 233, 511 (1984); L. Ibañez, C. López, C. Muñoz, Nucl. Phys. B 256, 218 (1985)

21. A. Dobado, M.J. Herrero, S. Peñaranda, in preparation
22. A. Dobado, M.J. Herrero, S. Peñaranda, "The $S M$ as the quantum low energy effective theory of the MSSM", FTUAM 98/8

23. G. Giavarini, C.P. Martin, F. Ruiz Ruiz, Nucl. Phys. B 381, 222 (1992)

24. An exact diagonalization in the charginos and neutralinos sector can be found in: M.M. El Kheishen, A.A. Shafik, A.A. Aboshousha, Phys. Rev. D 45, 4345 (1992); An approximate diagonalization can be found in: J.F. Gunion, H.E. Haber, Phys. Rev. D 37, 2515 (1988); B. Grinstein, J. Polchinski, M.B. Wise, Phys. Lett. B 130, 285 (1983)

25. M.E. Peskin, T. Takeuchi, Phys. Rev. Lett. 65, 964 (1990); Phys. Rev. D 46, 381 (1992) 